Florida International University FIU Digital Commons

3-29-2017

\title{
Scanning Ion Conductance Microscopy for Single Cell Imaging and Analysis
}

Namuna Panday

Florida International University, npand006@fiu.edu

DOI: $10.25148 /$ etd.FIDC001918

Follow this and additional works at: https:// digitalcommons.fiu.edu/etd

Part of the Biological and Chemical Physics Commons

\section{Recommended Citation}

Panday, Namuna, "Scanning Ion Conductance Microscopy for Single Cell Imaging and Analysis" (2017). FIU Electronic Theses and Dissertations. 3477.

https://digitalcommons.fiu.edu/etd/3477

This work is brought to you for free and open access by the University Graduate School at FIU Digital Commons. It has been accepted for inclusion in FIU Electronic Theses and Dissertations by an authorized administrator of FIU Digital Commons. For more information, please contact dcc@fiu.edu. 


\title{
FLORIDA INTERNATIONAL UNIVERSITY
}

Miami, Florida

SCANNING ION CONDUCTANCE MICROSCOPY FOR SINGLE CELL IMAGING AND ANALYSIS

\author{
A dissertation submitted in partial fulfillment of \\ the requirements for the degree of \\ DOCTOR OF PHILOSOPHY \\ in \\ PHYSICS \\ by
}

Namuna Panday 
To: Dean Michael R. Heithaus

College of Arts, Sciences and Education

This dissertation, written by Namuna Panday, and entitled Scanning Ion Conductance Microscopy for Single Cell Imaging and Analysis, having been approved in respect to style and intellectual content, is referred to you for judgment.

We have read this dissertation and recommend that it be approved.

Joong-Ho Moon

Wenzhi Li

Prem P Chapagain

Jin He, Major Professor

Date of Defense: March 29, 2017

The dissertation of Namuna Panday is approved.

Dean Michael R. Heithaus

College of Arts, Sciences and Education

Andrés G. Gil

Vice President for Research and Economic Development and Dean of the University Graduate School

Florida International University, 2017 
C Copyright 2017 by Namuna Panday

All rights reserved. 


\section{DEDICATION}

I dedicate this thesis to my parents. 


\section{ACKNOWLEDGMENTS}

First and foremost, I would like to sincerely thank my advisor, Professor Jin He for his continuous inspiration, support, insight, and encouragement. It has been a great honor to be his first PhD student, and I feel so lucky and proud of it. I am especially very grateful for his patience and mentorship during my $\mathrm{PhD}$. Under his guidance, I have got several training including in problem-solving, critical thinking, professional presentations and paper writing which will be valuable assets to my future career. He not only motivated me to learn various research skills but also provided excellent opportunity and platform to accomplish these skills. All his contributions of time, ideas, and funding are the key factors to make my PhD experience fruitful, and I am indebted to him.

I would also like to extend my special gratitude to Dr. Xuwen Wang who helped me as my mentor in the theoretical part of my research. His expertise on theoretical analysis of experimental results, careful guidance, and valuable suggestions in every group meeting was indispensable for this dissertation. He was always available to answer my questions. I am also grateful to my committee members, Professor Prem Chapagain, Professor Wenzhi Li and professor Joong-Ho Moon for their valuable suggestions and comments towards the successful completion of this dissertation. I feel very fortunate to have them not only as committee members but also as collaborators during this dissertation research. I would also like to express my sincere thanks to Dr. Lidia Kos for providing me excellent environment for the collaboration project. Her valuable suggestions and expertise on the cell biology helped me to design the living cell experiment, interpret the results and follow the right track. 
In addition, I also greatly appreciate Dr. Shuai Chan who helped me in my data analysis part as well as taught me LabVIEW which will be a great asset in my future career too. I owe special thanks to past group members Dr. Yuping Shan, Dr. Purushottam Tiwari and Dr. Gongming Qian for providing me training in the experimental skills as well as suggestions for my research projects. I would like to express my sincere gratitude to the all the current group members of Dr.He's research group Dr. Jie Pan, Popular Pandey, Jing Guo, Feng Chen and Qiushuang Ai for their kind support and assistance in research and other normal activities. I appreciate Xiaoshuang Li and Yong Myoung for their help and cooperation during my dissertation years.

My especial thanks go to electrical engineer Norman from electronic shop and mechanical engineer Zahid from Machine shop. They were so kind and always provided prompt help to facilitate my research obstacles. I would also like to thank whole physics department along with all the professors and department secretaries for their support.

I would like to acknowledge the NSF support (1454544) for my Research Assistantship. I would also like to thank University Graduate School at Florida International University for providing me Dissertation Year Fellowship Award which helped me during thesis writing and completing my research project.

I would like to express my deepest appreciation for the endless love and tremendous support from my parents. Without their continuous support, encouragement and inspiration my journey of $\mathrm{PhD}$ would not be possible. I would also like to thank all my family, relatives and friends for encouragement and care. At the end, I would like to thank my inspiring husband for his support in every step, even in my research field giving several theoretical explanations. 


\section{ABSTRACT OF THE DISSERTATION \\ SCANNING ION CONDUCTANCE MICROSCOPY FOR SINGLE CELL IMAGING \\ AND ANALYSIS}

by

Namuna Panday

Florida International University, 2017

Miami, Florida

Professor Jin He, Major Professor

Most biological experiments are performed on an ensemble of cells under the assumption that all cells are identical. However, recent evidence from single cells studies reveals that this assumption is incorrect. Individual cells within the same generation may differ dramatically, and these differences have important consequences for the health and function of the entire living body. I have used Scanning Ion Conductance Microscopy (SICM) for imaging and analysis of topographical change of single cell membrane, which is difficult to be revealed by optical microscopes. Morphological change in the fixed and live HeLa cell membrane during endocytosis of conjugated polymer nanoparticles was studied. Results demonstrated SICM is a powerful tool to study the interaction between nanoparticle and cell membrane during internalization of nanoparticles through the membrane. This research can improve our fundamental understanding of cellular behavior and will be helpful for drug delivery applications.

Based on conventional SICM, we have developed a novel method to simultaneous map the topography and potential distributions of the single living cells membranes. At the first step, multifunctional nanopipettes (nanopore/nanoelectrode) have been fabricated and 
characterized. To demonstrate the potential sensing capability and understand the mechanism, I measured the ionic current and local electric potential change during translocation of $40 \mathrm{~nm}$ charged gold nanoparticles. Our results reveal the capability of the multifunctional probe for the highly sensitive detection of the ionic current and local electrical potential changes during the translocation of the charged entity through the nanopore. From the potential change, we revealed the dynamic assembly of GNPs before entering the nanopore. The experimental results are also nicely explained by the finite element method based numerical simulation results.

At the second step, I have measured the surface potential of living cell membrane at selected locations. Very recently, I have obtained results to show that we can map the extracellular membrane potential distribution of the complicated living cell membrane with sub-micron spatial resolution. This new imaging technique can help biologist to explore the extracellular potential distribution of varieties of cells quantitatively.These studies will have impacts on several biomedical applications such as regenerative repair and cancer treatment. 


\section{TABLE OF CONTENTS}

CHAPTER

PAGE

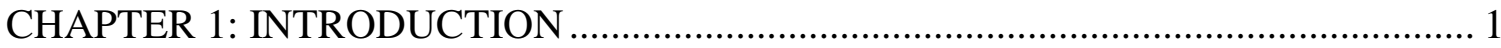

1.1: Motivation and Background to Study the Single Cell .............................................. 1

1.2: Scanning Probe Microscopy ………………………................................................ 2

1.2.1: Atomic Force Microscopy (AFM) ……….................................................. 2

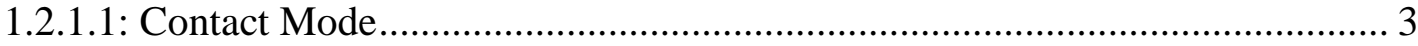

1.2.1.2: Non-Contact Mode: ........................................................................ 4

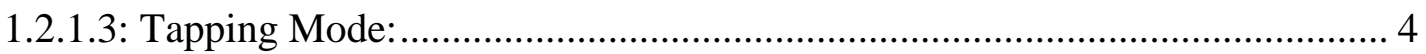

1.2.1.4: Phase Imaging:....................................................................................... 5

1.2.2: Scanning Ion Conductance Microscopy (SICM) ……....................................... 5

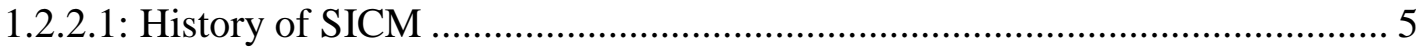

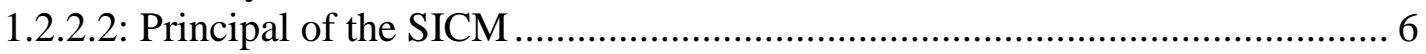

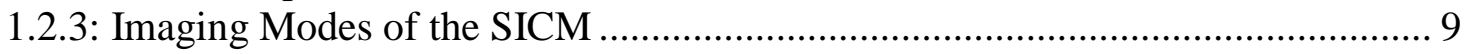

1.2.3.1: Direct Current (DC) Mode.................................................................. 9

1.2.3.1: Alternating Current (AC) Mode ............................................................... 10

1.2.3.3: Hopping Mode or Approach-Retract Scan (ARS) Mode.............................. 10

1.2.4: Advantages of SICM for the Living Cell Imaging ................................................ 11

1.3: Different Aspect of Live Cell Imaging With SICM ................................................. 13

1.3.1: Topography Imaging of Nanoparticles-Living Cell Membrane .......................... 13

1.3.2: Electrochemical Imaging of Living Cell Membrane ......................................... 15

1.4: Extracellular Potential Mapping of Living Cell Membrane ...................................... 19

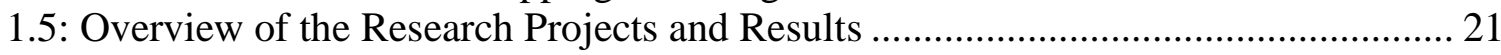

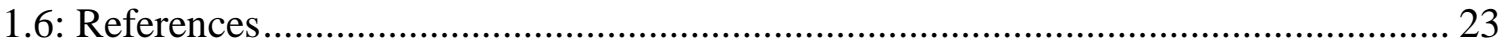

CHAPTER 2: METHODS AND MATERIALS …………….................................. 29

2.1: Characterization of Conjugated Polymer Nanoparticles (CPNs) With Atomic

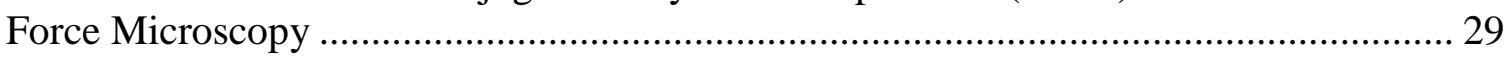

2.2: Scanning Ion Conductance Microscopy (SICM) for Single Cell Membrane

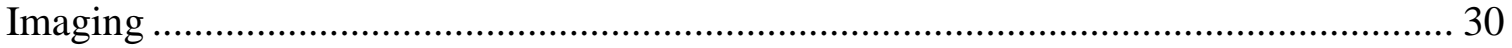

2.2.1: Nanopipette Fabrication................................................................................. 31

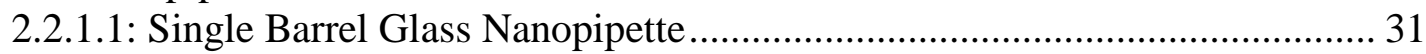

2.2.1.2: Double barrel quartz theta nanopipette ....................................................... 32

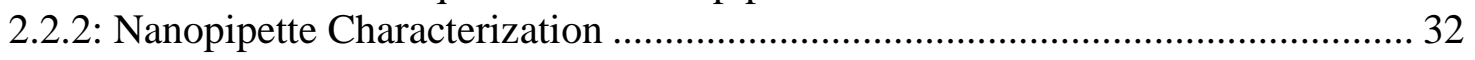

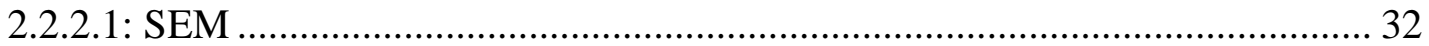

2.2.2.2: Measurement of pore diameter from the IV measurement .......................... 33

2.2.2.3: Optical microscope bright field imaging ..................................................... 35

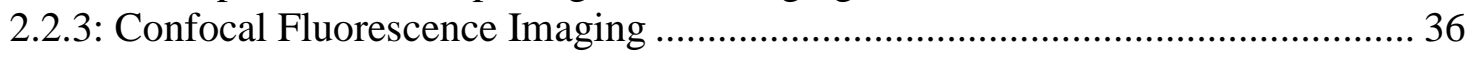

2.2.4: Data Collection and Analysis …………………............................................ 36

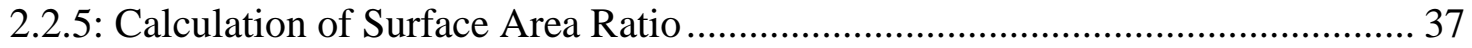

2.3: Multifunctional Scanning Ion Conductance Microscopy (SICM) ............................. 37

2.3.1: Pyrolytic Carbon Nanoelectrode Fabrication. .................................................... 38 
2.3.2: Characterization of Carbon-Nanoelectrode with Cyclic Voltammetry (CV)

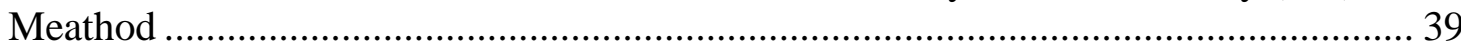

2.3.3: Measurement of Nanopore Surface Charge ......................................................... 41

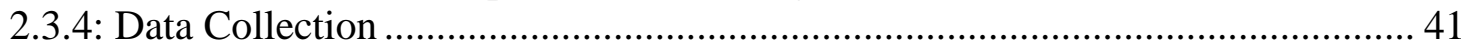

2.3.5: Zeta Potential Measurement of $40 \mathrm{~nm}$ GNPs with Dynamic Light Scattering

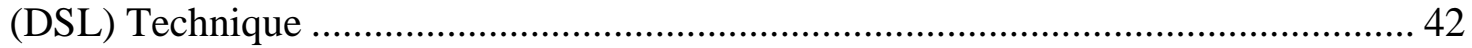

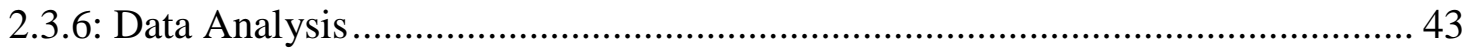

2.3.7: Noise Analysis of Ionic Current and Potential Measurements ........................... 43

2.3.8: Finite Element Based Numerical Simulations ..................................................... 44

2.4: Measuring Extracellular Potential Distributions of a Single Living Cell

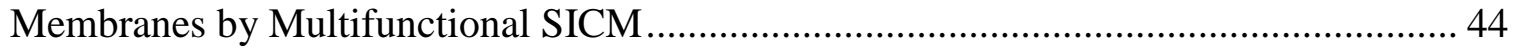

2.4.1: New Sample Stage for Living Cell Experiment ............................................. 44

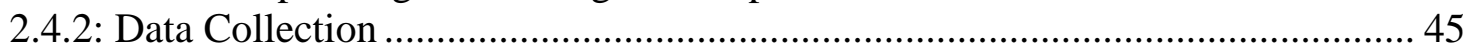

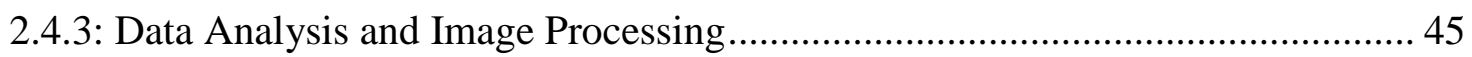

2.4.4: Finite Element Analysis of Potential Measurement. .............................................. 46

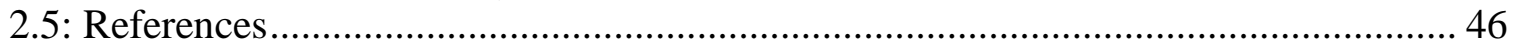

CHAPTER 3: CHARACTERIZATION OF THE POLYMER NANOPARTICLES

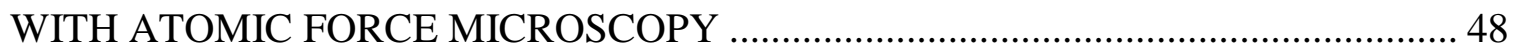

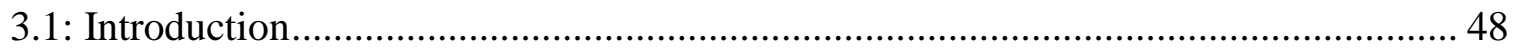

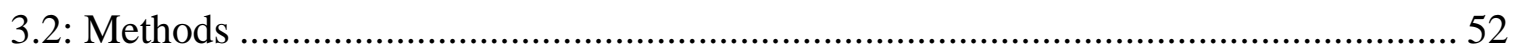

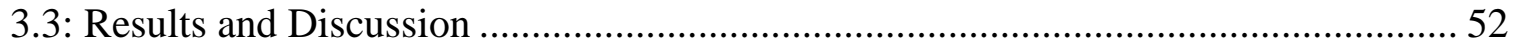

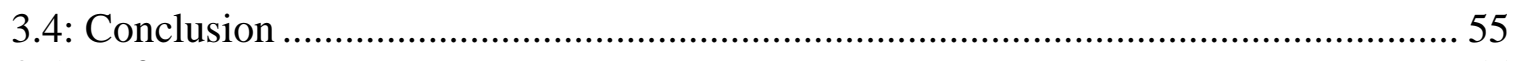

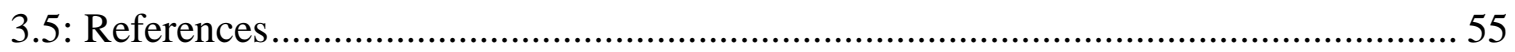

CHAPTER 4: SCANNING ION CONDUCTANCE MICROSCOPIC STUDY FOR CELLULAR UPTAKE OF CATIONIC CONJUGATED POLYMER

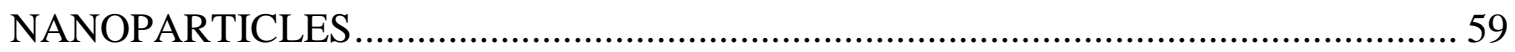

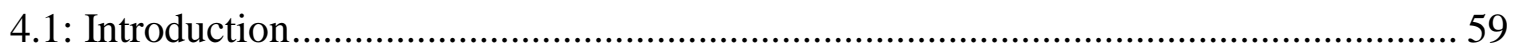

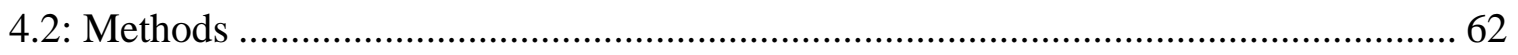

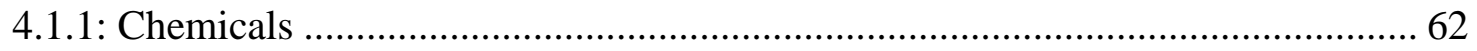

4.1.2: Cell Culture

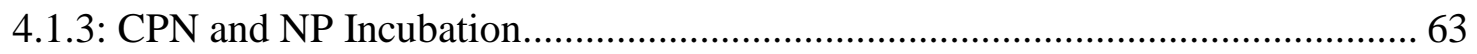

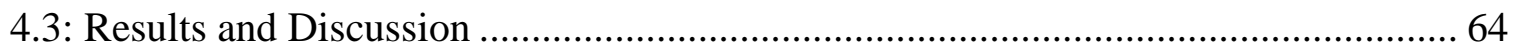

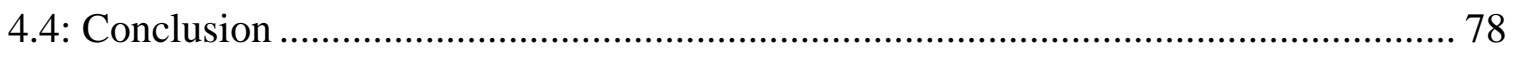

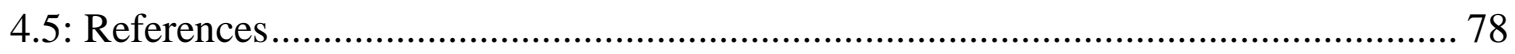

CHAPTER 5: SIMULTANEOUS IONIC CURRENT AND POTENTIAL DETECTION OF NANOPARTICLES BY A MULTIFUNCTIONAL

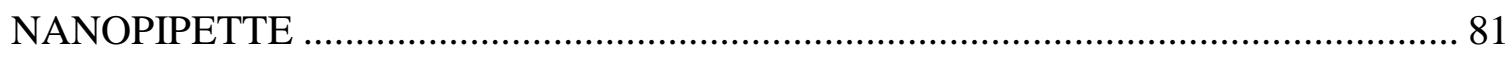

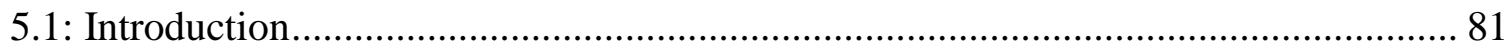

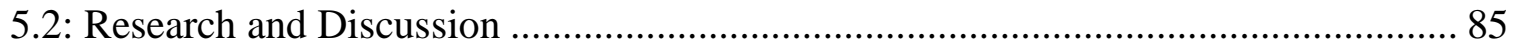

5.2.1: Multifunctional Nanopipette Fabrication and Characterization ......................... 85 
5.2.2: Simultaneous Ionic Current and Potential Measurements of GNP Motion........ 91

5.2.3: Optical Microscope Images of GNPs Inside the Nanopipettes After

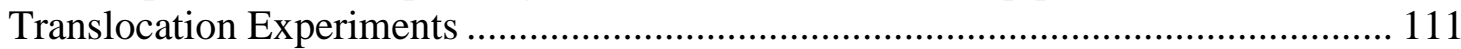

5.2.4: Noise Analysis of Ionic Current and Potential Measurements ....................... 112

5.2.5: Finite Element Based Numerical Simulations ................................................. 114

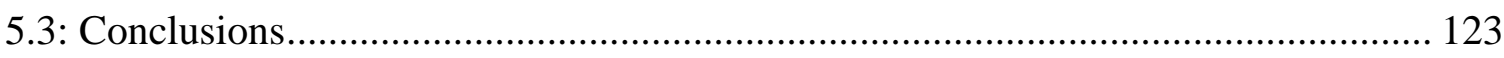

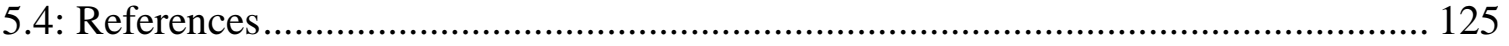

CHAPTER 6: MEASURING EXTRACELLULAR POTENTIAL DISTRIBUTIONS

OF A SINGLE LIVING CELL MEMBRANES BY SICM ....................................... 129

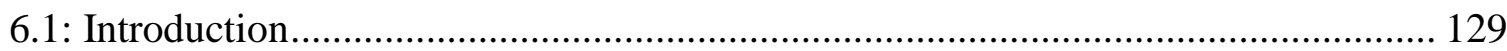

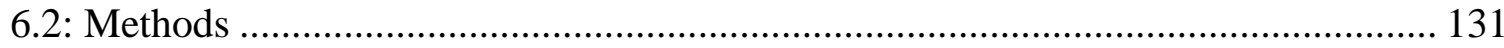

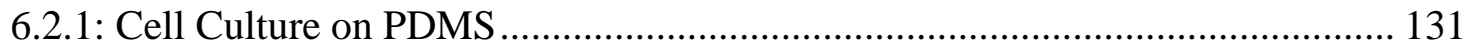

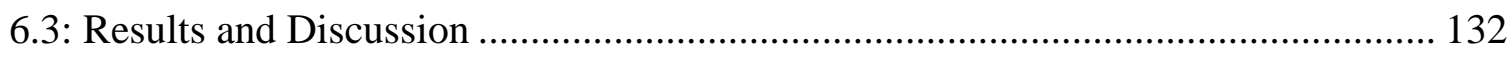

6.3.1: Single Point Potential Measurement.......................................................... 135

6.3.2: Potential Measurement by Mapping Certain Area of Living Cell ................... 138

6.4: Finite Element Based Numerical Simulations ..................................................... 140

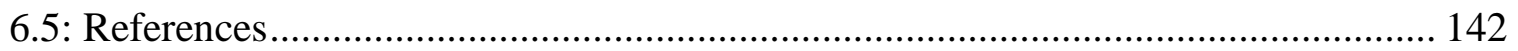

CHAPTER 7: SUMMARY AND FUTURE RESEARCH ...................................... 144

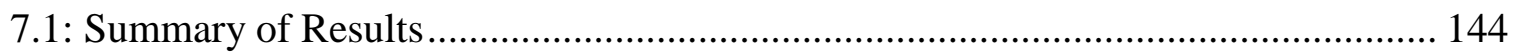

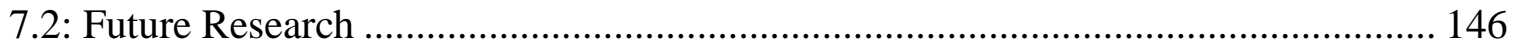

7.2.1: Potential and Topography Imaging of the Living Cell Membranes with

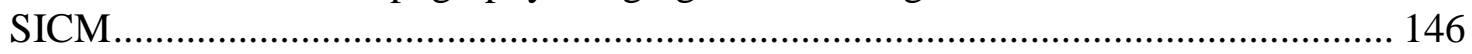

7.2.2: Potential Approach Curve Spectroscopy Analysis ...................................... 147

7.2.3: Finite Element Based Numerical Simulations .......................................... 147

7.3: Multifunctional Nanopipette for the Studies of Nanoparticles .............................. 147

7.4: Topography and Potential Imaging of Hole Formation on the Cell Membrane by

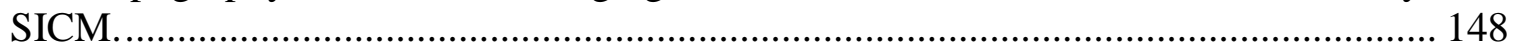

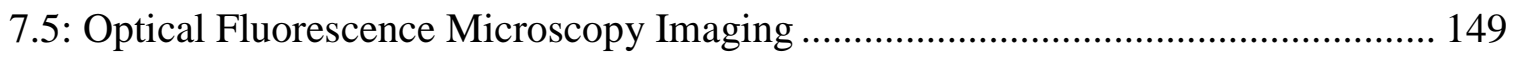

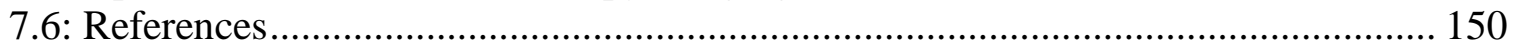

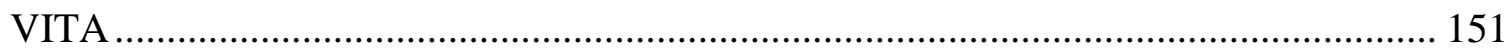




\section{LIST OF FIGURES}

FIGURES

PAGE

Figure 1.1: (a) Schematic of a Scanning Ion Conductance Microscopy. An electrolyte filled nanopipette is mounted on the Z- piezo and brought into the vicinity of the sample of interest, which is immersed in bath solution and fixed on an $x-y$ piezo stage and can be monitored by an inverted optical microscope. An applied bias between the bath electrode and pipette electrode generates ion current, and the current is used as feedback to control the pipette movement. (b) A typical approach curve (I-z curve) (black curve) and its fitting (red curve). $\mathrm{I}_{\mathrm{sp}}$ is the setpoint current, which is normally $2 \%$ lower than $\mathrm{I}_{0}$.

Figure 1.2: $\quad$ SECM/SICM hybrid technique. (a) The schematic of a typical SECM/SICM setup using a double-barrier carbon nanoprobe (DBCNP). (b) The Schematic of type one SECM/SICM probes with stripe structure (top) and ring structure (bottom) metal electrode respectively. The ring structure figure is reprinted with permission from ref 34 (copyright 2010, ACS Publishing Group).

Figure 2.1: The optical image of nanopipette located above living HeLa cell monolayer before performing scanning (scale bar $20 \mu \mathrm{m}$ ), the arrowhead points the location of nanopipette, which is much larger than the real size of the nanopipette tip.

Figure 2.2: $\quad$ SEM image of a typical glass nanopipette used for SICM. (a) Top view of the nanopipette (scale bar $100 \mathrm{~nm}$ ).(b) Side view of the nanopipette. The half cone angle is about 2 degree from the SEM image. The nanopipette was coated with about 5-6 nm thick gold for SEM imaging.

Figure 2.3(a) The IV curve of a typical glass nanopipette in 1x PBS buffer before approaching the nanopipette to the substrate surface. The arrow indicates the current normally used in SICM experiment. (b) The IV curve at small bias range $(-20 \mathrm{mV}$ to $+20 \mathrm{mV})$. The red curve is the linear fitting to the experimental data (black dots), and the pore resistance $\mathrm{Rp}=0.16 \mathrm{G} \Omega$ was obtained from 
the slope of the fitting line. (c) The Histogram of the pore resistance ( $\mathrm{Rp}$ ) of 10 nanopipettes. The red curve is the Gaussian fit, and the mean value is $0.16 \pm 0.01 \mathrm{G} \Omega$.

Figure 2.4: $\quad$ The optical images (a) a dual-nanopore nanopipette (before carbon deposition) where the black arrow indicates the separation between two barrels. (b) nanopore/CNE nanopipette (after carbon deposition) where the black arrow indicates the filled solid carbon only in one barrel.35

Figure2.5: The schematic of 1 by 1 pixel surface area calculation, the red spot indicates point's height, a gray area indicates the surface area, and green area indicates projected area.

Figure 2.6: The Schematic setup for the fabrication of CNE from the theta nanopipette

Figure 2.7: The steady-state CVs (at a sweep rate $20 \mathrm{mV} / \mathrm{s}$ ) for $18 \mathrm{CNEs}$ in $1 x$ PBS solution containing $1 \mathrm{mM} \mathrm{Ru}\left(\mathrm{NH}_{3}\right)^{6+}$ ions.

Figure 2.8: The schematic experimental setup of using nanopore/CNE nanopipette for simultaneous current and potential measurements of the GNP motion in the bath solution. $\mathrm{V}_{\mathrm{p}}$ is the applied bias. The potential is measured by a high impedance voltage meter.

Figure 2.9: Living cell imaging sample stage 45

Figure 3.1: (a) Chemical structure of the semiflexible PPB. (b) A schematic presentation of structural reorganization of the semiflexible PPB upon HA complexation.

Figure 3.2: (a) Chemical structure of PPE without flexible units along its rigid conjugated backbone. (b) Chemical structure of hyaluronic acid. 
Figure 3.3: AFM topographic $(a, b, c)$ and phase $(d, e, f)$ images of the semiflexible PPB (a,d), semiflexible PPB/HA nanoparticles formed at 1:1 molar ratio (b, e) and semiflexible PPB/HA nanoparticles formed at 1:3 molar ratio (c, f). The semiflexible $\mathrm{PPB} / \mathrm{HA}$ with 1:3 molar ratio exhibits elongated particles on a mica surface (c), and the phase image (f) reveals that the complexes are core-shell nanoparticles.

Figure 3.4: $\quad$ (a) Zoom in topography image of PPB/HA (1:3). (b) Profile of line 1 drawn above the nanoparticle on topography image (c) lateral size (d) height analysis histograms of semi-flexible PPB (red), semi-flexible PPB/HA (1:1) (green), and semi-flexible PPB/HA (1:3) (blue) respectively.

Figure 3.5: (a) Topography and (b) phase images, (c), size distribution and (d) height histograms for control PPE/HA.

Figure 3.6: (a) Topography image and (b) Phase image of CPN/HA(1:1)..........55

Figure 4.1: (a) The SICM image (enhanced color) of a live HeLa cells. (b-c) The SICM images (enhanced color) of live HeLa cells after treating with $10 \mathrm{uM}$ CPNs. (d) The histogram of the Protrusion height.

Figure 4.2: (a) The SICM topography image with the enhanced color of fixed HeLa cells without exposing to CPNs. (b) The SICM topography image of a zoom in area of a fixed HeLa cell membrane. $2^{\text {nd }}$ order flattening is applied to the image to remove cell contour.

Figure 4.3: (a) The chemical structure of CPN.(b-c) AFM topography images of CPNs. The images were taken in tapping mode in air. The CPNs were immobilized on the glass substrate (zoom-in image) in (c) or mica substrate (large scale image) in (b).

Figure 4.4: (a) The overlay of a single section confocal microscope bright field image and green channel fluorescence image at $5 \mu \mathrm{m}$ 
above the cell edge. The live HeLa cell was imaged after incubation with $10 \mu \mathrm{M} \mathrm{CPN}$ for 1 hour. (b) The fluorescence image of the red rectangular area in (a). (c) The 3D fluorescence image of the same cell in (a), which is composed of 33 images with $0.5 \mu \mathrm{m}$ resolution in $\mathrm{z}$-axis. The yellow arrow indicates the same location in (a). (d) The fluorescence image of the red rectangular area in (a).

Figure 4.5: (a) The SICM topography image with the enhanced color of HeLa cells treated with $10 \mu \mathrm{M}$ CPN for $1 \mathrm{~h}$. (b) A higher resolution image of the red rectangular area in Figure 3.5a. $2^{\text {nd }}$ order flattening was applied to the image to remove cell contour. (c) A high-resolution SICM topography image with the enhanced color of a small area of the cell surface. (d-e) The histograms of protrusion size and height (d), and pit size (e). The histograms are fitted by Gaussian functions. (f) The height profile across the blue dash line in Figure b. All the cells were fixed in $4 \%$ paraformaldehyde.

Figure 4.6: $\quad$ SICM topography image and height profile across the dashed line. One $1 \mu \mathrm{m}$ size polysterene (PS) bead with $1 \mu \mathrm{m}$ size can be identified (indicated by the arrow).

Figure 4.7: The distribution of SICM measured surface area ratio value of HeLa cells without (a) and with CPN (b), and with PEI (c) incubation at the same condition (10 $\mu \mathrm{M}$ for $1 \mathrm{~h})$.

Figure 4.8: $\quad$ SICM images of a typical PEI treated HeLa Cells. (A) Low resolution $(312 \mathrm{~nm} /$ pixel) SICM enhanced color topography image. (B) High resolution (39 nm/pixel) SICM topography image. (C) Enhanced color image of (B). The cells were treated by $10 \mu \mathrm{M}$ PEI for 1 hour and were fixed before imaging.

Figure 4.9: Confocal microscope bright field overlay images (a-b) and 3D fluorescence images (c-d) to show three HeLa cells after $4 \mathrm{~h}$ CPN incubation. 
Figure 4.10: (a) The incubation time dependence of cell surface area ratio for HeLa cell co-culture with $10 \mu \mathrm{M}$ CPNs. The surface area ratio magnitude was normalized by the maximum surface area ratio, which is indicated by the red arrow at $1 \mathrm{~h}$. Due to the large variation of surface area ratio, we only used data points above 30 for the mean value for time 15 minutes, 30 minutes and $1 \mathrm{~h}$. There are not enough data points above 30 for time $0,1.5 \mathrm{~h}, 2$ $\mathrm{h}$ and $4 \mathrm{~h}$, we still use all the data points for the mean value. (b) The maximum surface area ratio as a function of CPN concentration. (c) The time to reach maximum surface area ratio versus $\mathrm{CPN}$ concentration. The value is Mean $\pm \mathrm{SD}$ (standard deviation) in (b) and (c).

Figure 5.1: (a)The SEM image of the side view of a dual-nanopore nanopipette. The conical angle is measured using the two white guidelines. The left inset of (a) shows the top view of the dualnanopore nanopipette and two nanopores can be barely resolved. The top right inset of (a) shows the schematic of the dual-nanopore nanopipette where the black arrow indicates the thin layer separating two separate barrel. (b) The SEM image of the tip region of the nanopore/CNE nanopipette. Both nanopore and $\mathrm{CNE}$ can be resolved which has pointed by yellow arrows. The top right inset of (b) shows the schematic of the nanopore/CNE nanopipette. (c)The cross-section SEM image of a broken nanopore/CNE nanopipette. Before SEM imaging, the nanopipette was coated with a gold thin film (about $5 \mathrm{~nm}$ thick) to reduce charging during SEM imaging.

Figure 5.2: (a) IV curves of 28 nanopipettes before (green color) and after $\mathrm{CNE}$ fabrication (gray color). The IV curves were measured in $10 \mathrm{mM}$ PBS. The average curves are overlaid as bold red (before) and black (after) curves. (b) IVs in a small bias range for multifunctional nanopipettes $\mathrm{P} 1, \mathrm{P} 2$ and $\mathrm{P} 3$ used in the experiment. IVs were recorded in $10 \mathrm{mM}$ PBS ( $\mathrm{pH} 7.4$ ). The square symbol represents the experimental data and the straight lines are fitted lines. The black, red and green curves represent IVs for P1, P2 and P3 respectively. (c) The histograms of measured pore resistance (base on IV curves) before (green color) and after CNE fabrication (gray color). The solid lines are Gaussian fits to the histograms. The mean value is $2.22 \pm$ $0.53 \mathrm{G} \Omega$ before $\mathrm{CNE}$ fabrication and $3.00 \pm 1.16 \mathrm{G} \Omega$ after $\mathrm{GNE}$ 
fabrication. The error bar is the standard deviation of the Gaussian fits. (d) The histograms of rectification ratio $r$ before (green color) and after CNE fabrication (gray color). ). The solid lines are Gaussian fits to the histograms. The mean value is $-0.78 \pm 0.12$ before CNE fabrication and $-0.66 \pm 0.26$ after GNE fabrication. The error bar is the standard deviation of the Gaussian fits.

Figure 5.3: (a) CVs for multifunctional nanopipettes P1, P2 and P3 used in the experiment. $\mathrm{CVs}$ were recorded in $1 \mathrm{x}$ PBS solution containing $1 \mathrm{mM} \mathrm{Ru}\left(\mathrm{NH}_{3}\right)^{6+}$ ions at a sweep rate $20 \mathrm{mV} / \mathrm{s}$. The black, red and green curves represent CVs for P1, P2 and P3 respectively. (b) The distribution of CNE effective area.

Figure 5.4: (a) The current (black) and potential (red) traces (20 s) at $\mathrm{V}_{\mathrm{b}}=0.1 \mathrm{~V}$ before (i) and after (ii) adding 40nm GNPs in the bath solution. The potential dips at (iii), (iv), and (v) positions of time trace (ii) are also displayed in zoom-in traces. All the data are from nanopipette P1. Both bath and nanopore filling solution are $10 \mathrm{mM}$ PBS. Both the current and potential traces are collected at $50 \mathrm{kHz}$ sampling rate and smoothed using the moving average method with $0.2 \mathrm{~ms}$ ( 10 points) window size for current and $2 \mathrm{~ms}$ (100 points) window size for potential. (b) A scheme to show the translocation of single GNP in three steps. The red color region represents the potential sensing zone. (c) The proposed equivalent circuit of the nanopore/CNE system.

Figure 5.5: (a-b) The 20 seconds current (black), potential (V, red) and first derivative of potential traces (dV/dt, blue) at $0.1 \mathrm{~V}$ (top) for P1(a) and P2 (b) after adding $40 \mathrm{~nm}$ GNPs in the solution. The purple strips indicated the full time span of potential dips. The green arrows indicate three current spikes appeared during the potential decrease stage of a potential dip. (c-d) The zoom-in traces of the green shaded regions of (a) and (b). The red arrows mark the small potential changes. $0.2 \mathrm{~ms}$ (10 points) moving average is applied to both current and potential data. (e) A scheme to show the accumulation of GNPs near the nanopipette 
tip and the possible wire cluster formation of GNPs before translocation.

Figure 5.6: The scatter plot and histograms of potential dip height $\Delta \mathrm{V}_{\mathrm{p}}$ and width $\Delta \mathrm{t}_{\mathrm{p}}$ from 259 potential dips similar to (iii) and (iv) of 4.4 (a). The solid lines of the histograms are Gaussian fits.

Figure 5.7: (a-b) The intensity plots of the distributions of height and width of the potential steps (a) and corresponding current spikes (b) of P1 nanopore and CNE data. The yellow (at 1ms) and green dash lines are guides to the eye. (c) Two representatives (i) fast and (iii) slow translocation events with the potential steps (red color) and corresponding current spikes (black). The green dash lines indicate the start and end points for potential steps and current spikes. $0.2 \mathrm{~ms}$ (10 points) moving average is applied to both current and potential data.

Figure 5.8: (a) The setup for the measurement using dual-nanopore nanopipette. (b) The current (black) and potential (red) time traces (1s) before adding GNPs $(\mathrm{Vp}=0.5 \mathrm{~V})$. (c) The current (black) and potential (red) time traces (1s) after adding GNPs $(\mathrm{V} p=0.5 \mathrm{~V})$. (d) The zoom-in trace of the green color shaded region to show individual rectangular shape current spikes and the corresponding potential change.

Figure 5.9: The histograms for spike height $\left(\Delta \mathrm{I}_{\mathrm{I}}\right)$ and spike width $\left(\Delta \mathrm{t}_{\mathrm{I}}\right)$ of 583 current spikes with dual nanopore nanopipette. The red curves are the Gaussian fits.

Figure 5.10: Optical microscope images for (i) dual-nanopore nanopipette and (ii) $\mathrm{CNE} /$ nanopore nanopipette after GNP translocation experiments. The GNP aggregates are clearly visible inside the nanopipette barrel.

Figure 5.11: $\quad(a, b)$ The normalized noise power spectra for ionic current (a) and potential (b) for a 1 second time trace from nanopipettes 
$\mathrm{P} 1, \mathrm{P} 2$ and $\mathrm{P} 3$ at applied bias $\mathrm{V}_{\mathrm{b}}=100 \mathrm{mV}$. No GNPs were added in the solution. (c) The normalized potential noise power spectra for P1 before (blue) and after adding GNPs in the solution at various times. A higher bias $\left(\mathrm{V}_{\mathrm{b}}=300 \mathrm{mV}\right)$ is used during GNP accumulation. (d) The normalized potential noise spectra for P1, P2 and P3 at $\mathrm{V}_{b}=100 \mathrm{mV}$ after GNP accumulation. (e) A typical curve of $\mathrm{P} 1$ at $50 \mathrm{mV}$ after adding GNPs. The black curve is the raw data for current measurement and the red curve is the smoothed curve of current after 10 points moving average. Similarly, the gray curve is the raw data for potential measurement and the blue curve is the smoothed curve of potential after 10 points moving average. (f) The zoomed in current and potential traces after applying moving average smoothing method. Both raw data and smoothed data are shown. The sampling rate is $50 \mathrm{kS} / \mathrm{s}$ for both measurements and the bandwidth is $5 \mathrm{k} \mathrm{Hz}$ for current and $40 \mathrm{k} \mathrm{Hz}$ for potential. The bath solution is always $10 \mathrm{mM}$ PBS.

Figure 5.12: The 2D axial symmetric geometry of the nanopipette/CNE used for the FEM simulations. The figures are drawn to scale ( $\mathrm{r}=0$ indicates the axis symmetry line). Insets: (i) the quasi $3 \mathrm{D}$ view of the simulation model near the tip. (ii-iii) zoom-in views of the tip region with (ii) a hemispherical shape $\mathrm{CNE}$ with radius $38.5 \mathrm{~nm}$ and (iii) a flat CNE. (iv) mesh distribution near the tip of hemispherical shape CNE.

Figure 5.13: $\quad$ FEM simulation results (a) The Electric field distribution near the nanopipette tip when a GNP is at the nanopore orifice $(\mathrm{Z}=1 \mu \mathrm{m}, \mathrm{r}=0 \mathrm{~nm})$. The white arrows indicate the direction of the field, and the color bar shows the intensity of the field. The axis symmetry line is at $r=0$. (b) The distributions of potential (top) and $\mathrm{Z}$ component electric field ( $\mathrm{Ez}=\mathrm{dV} / \mathrm{dZ}$ ) (bottom) along the yellow dash line showing in (a). Both plots contain five distributions with a GNP at five different locations (indicated by the red arrows). The inset in potential distribution shows the small change of the potential distribution when the GNP is near the nanopore entrance ( $\mathrm{Z}=0.9 \mu \mathrm{m}, \mathrm{r}=0 \mathrm{~nm})$. (c) The ionic current (black) changes of the nanopore and potential (red) change at the CNE as a function of GNP center position in $\mathrm{Z}$ direction along the axis symmetry line. (d) The evolution of the $\Delta \mathrm{V}-\mathrm{Z}$ plots as the surface charge density of the GNP 
increase. The surface charge densities at the surface of quartz, $\mathrm{CNE}$ and GNP are $-5 \mathrm{mC} / \mathrm{m}^{2}, 0 \mathrm{mC} / \mathrm{m}^{2}$ and $-24 \mathrm{mC} / \mathrm{m}^{2}$ respectively, if not mentioned otherwise.

Figure 5.14: (a) Electric potential distribution near the tip region. (b) The net ion distribution near the tip region. Only potassium and chlorine ions are considered in the simulation. (c) The potential changes versus the GNP center position along $\mathrm{Z}$ axis at different GNP and CNE polarization conditions. (d) The potential changes versus the GNP center position along $\mathrm{Z}$ axis with CNE geometry (ii) and (iii) in (6.1). (e) The zoom-in of (d) near the nanopore mouth. (f) The potential changes versus the GNP center position along $\mathrm{Z}$ axis with one (black) and three GNPs (red) at the nanopore mouth. For 3 GNPs case, the $Z$ position is the center of the first GNP. The inset is the electric field distribution for 3 GNPs.

Figure 6.1: $\quad$ (a) Schematic of a potentiometric SICM (P-SICM) setup using the theta nanopipette as a probe. (b) Equivalent circuit model of the experimental setup.

Figure 6.2: (a) simultaneously acquired approach curves for ionic current and the potential difference with potentiometric SICM. (b) Approach /retract curve spectroscopy on positive and negative charged substrate. 134

Figure 6.3: Extracellular membrane potential recorded by P-SICM with fixed point measurement technique. Histograms of extracellular membrane potential of (a) Melanoma (B16) (red) and melanocytes (Mela-A) (blue) recorded in $45 \mathrm{mM}$ outside potassium concentration.(b)melanocytes (Mela-A) recorded in $5 \mathrm{mM}$ (blue) and $45 \mathrm{mM}$ (green) outside potassium concentration. (c) melanoma (B16) recorded in $5 \mathrm{mM}$ (red) and $45 \mathrm{mM}$ (green) outside potassium concentration. The red, blue and green solid lines are Gaussian fits to the histograms. The mean value was $0.5 \pm 0.1 \mathrm{mV}$ and $0.9 \pm 0.3 \mathrm{mV}$ for Mela-A and $\mathrm{B} 16$ cells respectively in $45 \mathrm{mM}$ outside $\mathrm{kcl}$ concentration. The mean value became $1.4 \pm 0.4 \mathrm{mV}$ and $1.1 \pm 0.4 \mathrm{mV}$ for Mela- 
$\mathrm{A}$ and $\mathrm{B} 16$ respectively in $5 \mathrm{mM}$ outside $\mathrm{kcl}$. .The error bar is the standard deviation of the Gaussian fits.

Figure 6.4: $\quad$ Simultaneous recorded (a) topography and (b) potential image of living melanoma cell with P-SICM. (c) Enhanced color topography image (d) Zoom in the image of the potential image inside a rectangular area.

Figure 6.5: $\quad$ FEM simulation result of measured potential with respect to the vertical position of the potential probe from different surface charged substrate. Figure inset is the zoomed in the plot of the original plot.

Figure 7.1: (a) Enhanced color topography image of fixed HeLa cell treated with the $10 \mu \mathrm{M}$ guanidine for $1 \mathrm{hr}$. (b) Zoomed in images of the Figure (a) enclosed inside the red rectangular area. (c) The structure of the guanidinium CPN where red circle focuses the side chain. (d) The height profile across the red solid line in Figure $b$. The cell was fixed in 4\% paraformaldehyde. 149 


\section{CHAPTER 1: INTRODUCTION}

\section{1: Motivation and Background to Study the Single Cell}

Biological cells are fundamental building blocks of every living being. They are very complex in nature. Many biological experiments are performed on groups of cells under the assumption that all cells of a particular type are identical. However, recent evidence from studies of single cells reveals that this assumption is incorrect. ${ }^{1}$ Individual cells within the same generation may differ dramatically, and these differences can have important consequences for the health and function. Even in the same types and same generation of cells, there exists significant differences in cell morphology as well as patterns of extracellular membrane potential distributions at different stages of the cell cycle. On the living cell membrane, there are multiple microdomains of membrane potential induced by systematically distributed ion channels and pumps. ${ }^{2}$ These systematically distributed ion channels lead to physiological micro-domains around an individual living cell or a cluster of cells. Recently, the existence of multiple microdomains of extracellular membrane potential around individual cells have been explored by voltage

reporter dye using fluorescence microscopy. ${ }^{2-4}$ The new information of these patterns of long lasting extracellular membrane potential plays a vital role in regulating important cell activities such as embryonic patterning, regenerative repair, and reduction of cancerous disorganization. ${ }^{5}$

To better understand single cell behaviors and the dynamic patterns of extra cellular membrane potential, it is necessary to acquire the details of these membrane potentials with high spatial resolution and reasonable time resolution $(\sim \mathrm{ms})$. Although fluorescence microscopy allows real-time monitoring of a variety of extracellular and intracellular 
bioelectric signals at the single cell level ${ }^{2,3,6}$, its spatial resolution is still not enough to resolve the subcellular structures and its signals. Furthermore, fluorescence microscopy images cannot give the quantitative measurement of the extracellular membrane potential. In addition to Fluorescence microscopy, several other techniques have also been used to understand bioelectric signals at the cell membrane. In the last two decades, scanning probe microscopy techniques mainly, Atomic Force Microscopy (AFM) and Scanning Ion Conductance Microscopy (SICM) have been used for imaging and analysis of the biological specimens. However, SICM is preferred to AFM for imaging and analysis of fragile and complex live cell membranes because SICM can take the images of the samples without touching on its surface with comparable resolution with AFM.

\section{2: Scanning Probe Microscopy}

Scanning probe microscopy (SPM) is a branch of microscopy which uses a physical probe to form images by raster scanning over of the specimen. The first SPM was invented in 1981 by Gerd Binnig and Heinrich Rohrer for imaging surfaces at the atomic level. After the invention of STM, many variations of probe based microscopy have been developed. Among them, atomic force microscopy (AFM) and scanning ion conductance microscopy (SICM) are widely applied SPM techniques in the field of nanobiophysics.

\subsection{1: Atomic Force Microscopy (AFM)}

Atomic force microscopy (AFM) is very versatile and powerful scanning probe microscopy technique for studying different types of samples such as thin and thick film coatings, ceramics, composites, glasses, synthetic and biological membranes, metals, polymers, and semiconductors at the nanoscale. ${ }^{7}$ It is versatile because an AFM not only generates three-dimensional topography images with angstrom scale resolution, but it also 
reveals various types of surface properties. ${ }^{8-11}$ Furthermore, it has been widely applied to investigate the properties of single molecules such as DNA, protein complexes, and nanoparticles as well as to detect intermolecular forces. ${ }^{12-16}$ An AFM uses a cantilever with a very sharp tip at its end which is used to scan over a sample surface. As the tip approaches the sample surface, the force between the tip and the sample results into a deflection of the cantilever. The deflection of the cantilever reveals the surface topology of the sample with nanometer precision. It can be operated in both air and liquid environment. However, for the biological samples, liquid imaging is performed because imaging biological samples in liquid environment help them to provide the physiological environment. The AFM has three standard imaging mode which is described in brief in below.

\subsubsection{1: Contact Mode}

In contact mode AFM, the tip scans over the sample surface with direct contact. The contact force (repulsive force) during imaging causes the cantilever to bend. As the cantilever bend, the position of the laser beam focused on the back of the cantilever changes which is detected by the position sensitive photodiode. Once cantilever deflection is detected by photodiode, the true topographic image is generated. Topography images in the contact mode are acquired in either constant height mode or constant force mode. In constant height mode, the height of the scanner is fixed, and the variation of the cantilever deflection is directly recorded as a topography image of the sample. The constant-height mode is useful for taking atomic-scale images of atomically flat surfaces, where the variation of force between the tip and the sample are small. In constant force mode, the cantilever deflection is maintained constant by moving the scanner up and down according 
to the surface features. The movement of the scanner in the $\mathrm{z}$ direction is recorded as the topography image. The constant force mode is preferred for most of the applications.

Because of the continuous contact between the tip and the sample, there is always a risk to damage the sample as well as the tip. The contact mode imaging is only good for the hard surface. But the image resolution is very high in contact mode in comparison tapping mode and non-contact mode.

\subsubsection{2: Non-Contact Mode:}

In non-contact mode, the tip vibrates above the sample with its resonance frequency by keeping certain distance $(50 \AA-150 \AA)$ between the tip and the sample surface. Attractive Van der Waals forces acting between the tip and the sample are detected and used to generate the topography image of the sample. Since the attractive Van der Walls force is much weaker than the repulsive contact mode force, the image resolution is

not very good. Furthermore, in the liquid mode, the range of the Van der Walls force is dominated by the liquid contamination layer which makes the imaging very difficult. However, there is no risk of sample damage because of the constant interaction between the tip and sample surface like in contact mode. Hence this mode is preferred for the soft samples like biological samples.

\subsubsection{3: Tapping Mode:}

Tapping mode AFM was developed to combine the advantages of contact mode and non-contact mode imaging. In tapping mode, the cantilever is oscillated above the sample surface nearly or exactly at its resonant frequency with an amplitude in the range of $10-100 \mathrm{~nm}$. The feedback loop is set to make the constant amplitude of the cantilever 
oscillation. Hence, the oscillating cantilever records the topography features of the sample by contacting the sample surface intermittently and very gently. With the tapping mode technique, the very soft and fragile biological samples can be imaged successfully with high resolution.

\subsubsection{4: Phase Imaging:}

Phase imaging is another advantage of tapping mode which can be recorded simultaneously with topography image. The phase image is generated by mapping the phase shift of the cantilever oscillation during tapping mode scanning. It is a powerful approach to imaging that provides extra information about surface structure with a nanometer-scale resolution which often not revealed by other SPM techniques. It can give more information than the simple topographical mapping to detect variations in composition, adhesion, friction, viscoelasticity, and so on. Phase imaging can be applied to the mapping of different components in a complex material, identification of contaminants and differentiation of hard and soft region.

\subsection{2: Scanning Ion Conductance Microscopy (SICM)}

\subsubsection{1: History of SICM}

After the introduction of scanning tunneling microscopy (STM) ${ }^{17-19}$ in 1981 and atomic force microscopy (AFM) ${ }^{20}$ in 1985 by Binnig and Rohler, many variations of scanning probe based microscopes have been developed. ${ }^{21}$ Scanning ion conductance microscopy (SICM) is one of them, which was originally designed by Hansma and coworkers in 1989 for imaging nanoporous and non-conductive polymer membrane ${ }^{22}$ and later applied to image soft living cell membranes under physiological conditions by 
Korchev's group. ${ }^{23}$ SICM is a unique combination of patch-clamp ${ }^{24}$ and SPM technique. ${ }^{23}$ The development of SICM is driven by several active research areas, including Biophysics, electrophysiology, electrochemistry and nanotechnology. ${ }^{25-28}$ As a result of the improvement in feedback control system in recent years, SCIM has emerged as a powerful and reliable imaging and analytical tool to noninvasively study living cells with high spatial resolution under physiological conditions at different levels of organization (tissue, cellular and subcellular levels). In the early days, all the SICM units were home-built. At the time of writing this dissertation, SICM is now commercially available and can be purchased from Ionscope and Park Systems. The SICM setup is often housed on an inverted optical microscope so that the tip approach process can be guided optically. The detailed description of the principle of SICM and various feedback mechanisms is discussed in the following sections.

\subsubsection{2: Principal of the SICM}

A typical setup of SICM is shown in Figure 1.1. An electrolyte-filled glass or quartz pipette is used as the probe to raster scan the targeted surfaces immersed in the electrolyte with high spatial resolution. A pipette puller can easily and reproducibly prepare the nanopipette with tens of nanometer opening at the tip. A potential difference is applied between the pipette electrode (which is inserted inside the pipette) and the bath electrode (immersed in the bath solution). The electrodes are normally silver/silver chloride electrodes. Because of the applied potential bias between these two electrodes, steady state ion current $I_{0}$ flows through the nanopipette which is mainly determined by the inner diameter of the nanopipette when the pipette is far from a sample of interest. When the 
nanopipette tip approaches close to the sample surface of interest, the ionic current through the nanopipette $I_{0}$ is strongly affected by the access resistance $\left(R_{a c}\right)$, which is a function of the distance $\mathrm{z}$ between the nanopipette tip and the surface. Hence, the magnitude of ion currents $I_{0}$ depends on the applied bias and the total resistance of the pipette $\left(R_{T}\right)$, which includes the resistance of the pipette $\left(R_{p}\right)$, and the access resistance between the pipette tip and the sample surface $\left(R_{a c}\right)$, which can be described mathematically by following equation $1.1 .^{29}$

$I(Z)=\frac{V}{R_{T}}=\frac{V}{R_{p}+R_{a c}}$

The pipette resistance $R_{p}$ can be calculated geometrically by following equation 1.2

$R_{p}=R_{\text {ext }}+R_{\text {int }}$

Where $R_{\text {ext }}=\frac{1}{4 k r_{i}}$, is the external resistance of the nanopipette, and

$R_{\text {int }}=\frac{1}{\pi k r_{i} \tan \theta}$, is the internal resistance of the nanopipette, where $r_{i}$ is the inner radius of the nanopipette tip opening, $k$ is the conductivity of the electrolyte solutions and the $\theta$ is the half cone angle of the nanopipette. Equation 1.2 can be rewrite as follow,

$R_{P}=\frac{1}{4 \mathrm{k} r_{i}}+\frac{1}{\pi k r_{i} \tan \theta}$

For our nanopipette, the half cone angle $\theta$ is very small. When $\theta$ is small we can ignore the first term of the equation 1.3 and $R_{P}$ can be calculated from the following equation 1.3. $R_{P} \approx \frac{1}{\pi k r_{i} \tan \theta}$

The pipette resistance $R_{P}$ is always constant for the pipettes having same geometry. Although pipette resistance $R_{P}$ is constant for pipettes with the same geometry, access resistance $R_{a c}$ is strongly affected by the distance $\mathrm{z}$ between the nanopipette tip and the 
sample. A mathematical description of $R_{a c}$ is given by Equation 1.5, where $r_{o}$ is the outer radius of the nanopipette tip opening.

$R_{a c c} \approx \frac{\frac{3}{2} \ln \frac{r_{O}}{r_{i}}}{\pi k z}$

Now, equation 1.1 can be written as following which clearly shows the dependence of ion currents on probe sample distances.

$I(Z) \approx I_{0}\left(1+\frac{B}{Z}\right)^{-1} ; I_{0}=\frac{V}{R_{p}}$

Where $B=\frac{3}{2} \ln \left(\frac{r_{o}}{r_{i}}\right) r_{i} \tan \theta$ is the geometric factor.

The relationship between $I_{0}$ and distance $\mathrm{z}$ can be understand from the approach curve. Actually approach curve is the current versus distance curve which is recorded during the approaching of the nanopipette towards the sample surface. When the nanopipette tip is far from the sample, there is a constant maximum current $I_{0}$, but when it gradually approaches to the sample surface, there is a sharp decrease in the ionic current with the distance. When the nanopipette tip approaches near a sample surface, the ion current pathway is affected by the sample surface and becomes sensitive to the vertical distance between the nanopipette tip and the sample surface, which can be used as a feedback signal to control the nanopipette height above the sample surface. A typical approach curve is shown in the figure $1.1(\mathrm{~b})$ which can be fitted by, $I(Z) \approx I_{0}\left(1+\frac{B}{Z}\right)^{-1}$ where B is a geometry constant and $I_{0}$ is the current when the nanopipette is far away from the surface. Since $I(Z)$ is a function of $z$, it has been used as the feedback signal to control the vertical position of the pipette during the imaging. By recording the vertical motion of the pipette as it follows the sample surface, one can generate a topographic image. 

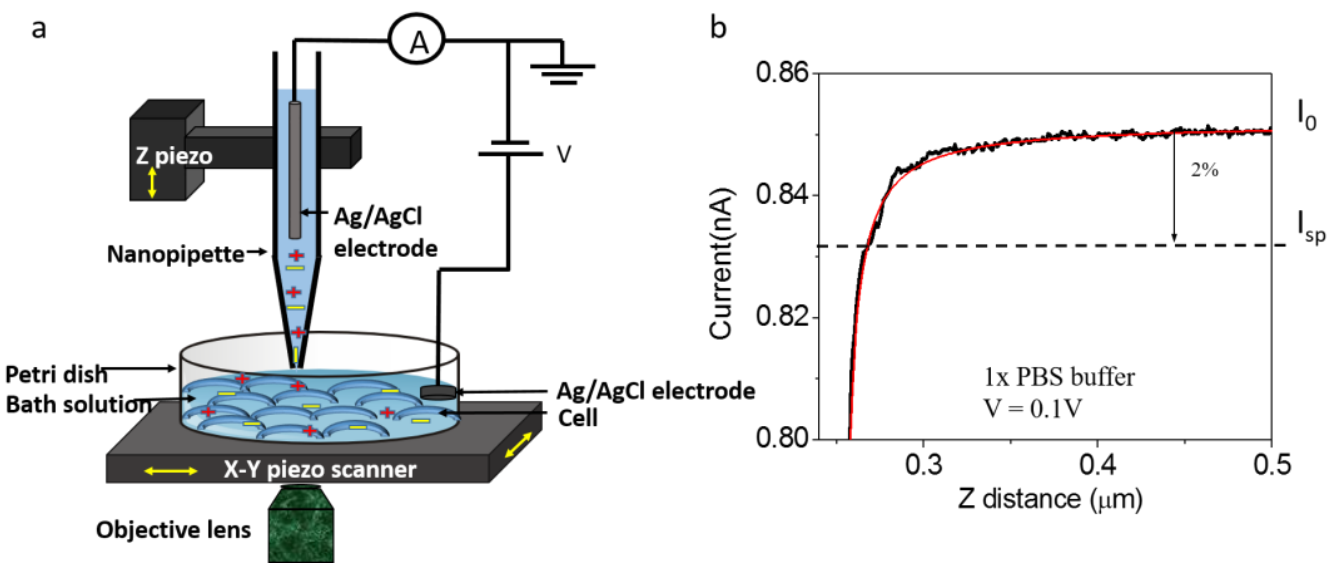

Figure 1.1: (a) Schematic of a Scanning Ion Conductance Microscopy. An electrolyte filled nanopipette is mounted on the Z- piezo and brought into the vicinity of the sample of interest, which is immersed in bath solution and fixed on an x-y piezo stage and can be monitored by an inverted optical microscope. An applied bias between the bath electrode and pipette electrode generates ion current, and the current is used as feedback to control the pipette movement. (b) A typical approach curve (I-z curve) (black curve) and its fitting (red curve). $\boldsymbol{I}_{\boldsymbol{s p}}$ is the setpoint current, which is normally $2 \%$ lower than $\boldsymbol{I}_{\mathbf{0}}$.

\subsection{3: Imaging Modes of the SICM}

If we examine the difference in feedback mechanisms, there are three imaging modes for SICM: direct current (DC) mode, alternating current (AC) mode and hopping mode (or approach-retract (ARS) mode). I describe each imaging mode as follows.

\subsubsection{1: Direct Current (DC) Mode}

Direct current (DC) mode is also known as constant distance mode. In this mode, the nanopipette is lowered toward the sample until a predefined setpoint current is achieved. The pipette is then moved over the contours of the surface by maintaining the ionic current constant through continuous adjustment of the $\mathrm{z}$-axis. ${ }^{30}$ The z-position of the pipette determines the topography of the sample. The image resolution is highly dependent on the nanopipette size. The mode is only applicable to very flat samples because it does not detect steep slopes in the uneven sample. During imaging, nanopipette may contact the 
sample and break the nanopipette tip. There is also the very high risk of the electrode drift and system may lose the feedback control after a long time of scanning.

\subsubsection{1: Alternating Current (AC) Mode}

In alternating current (AC) mode, the nanopipette oscillates vertically on the sample surface along with its usual movement. When the nanopipette is far from the sample surface, there is the constant steady current like in the DC mode, so the nanopipette is lowered towards the sample surface. When the height of the nanopipette on the surface starts to modulate in the z-direction, the modulated current wave is generated which is used as a signal for the feedback mechanism to control the height of the nanopipette. ${ }^{31}$ Image resolution also depends on the pore size of the nanopipette as in DC mode. However, AC mode can apply to the more complex samples than the DC mode with less electrode drift. $\mathrm{AC}$ mode can be used for the real-time monitoring of the samples with higher resolutions.

\subsubsection{3: Hopping Mode or Approach-Retract Scan (ARS) Mode}

Hopping mode SICM is developed to scan very complex surface features such as

neuron cells. ${ }^{32,}{ }^{33}$ There is no continuous feedback mechanism to control the pipette position above the sample as in DC and AC mode. In the hopping mode, nano-pipette approaches the sample surface only at the selected imaging points. A different reference current point is set at each selected imaging points. As the pipette approaches towards the sample, the current drops rapidly. When current drop reaches a predefined value, so called the set-point value, the z-axis position of the pipette is recorded, and the z-piezo withdraws the pipette from the surface. At each imaging point, a reference current is also measured when the pipette is far from the sample surface. The sample is then moved to a next imaging 
point by the $x-y$ piezo, and the same cycle starts again. The image resolutions and total time for the imaging are mainly determined by the number of imaging points. In comparing to DC and AC imaging mode, this mode can take very high-resolution images of the highly contoured biosamples. Hopping mode is normally used for cell imaging. The major drawback of hopping mode is the slow speed.

The lower resolution image of the sample of interest is acquired at first and then image more complex areas of the sample with higher resolution to reduce the imaging time. ${ }^{21}$ In hopping mode, to expedite the imaging, the whole scan area is subdivided into the equal squared shaped areas (represented by pixel x pixel). The roughness of the every area is estimated by measuring the height difference at each corner. If the average roughness of the squared area is high, the higher resolution image is acquired. But, if the average roughness of the squared area is low, lower resolution image is acquired. The withdrawn height of the nanopipette also can be adjusted just above the predetermined roughness of each square area. Hence, it can save the time by preventing withdraw of the nanopipette all the way up.

Recently, Korchev's group have developed a fast speed hopping mode SICM by using the fast shear piezo-actuator which has the capability to track the dynamic interactions between a single nanoparticle and a single cell. ${ }^{25}$

\subsection{4: Advantages of SICM for the Living Cell Imaging}

There are several advantages of using SICM to study living cells in comparison to the other SPM techniques. The SICM enables noninvasive topography image of living cell membrane in their native environment with tens of nanometer scale resolution. The SICM has been applied to investigate important cell activities at the subcellular and cellular 
levels, including dynamic changes in plasma membrane morphology associated with endocytosis and exocytosis, ${ }^{34,35}$ and dynamics of cellular surface assembly of living cells. 36, 37 SICM has also resolved the location, structure and dynamics of single protein and protein complex in the cell membrane. ${ }^{38,39}$ Researchers have been demonstrated that the SICM can track the membrane structure changes of the same cell up to several days in an environment control chamber. ${ }^{36,37,40,41}$ The long term study will also be extremely helpful for observing slow cell activities, such as cell migration and volume changes, ${ }^{42}$ in real time. Another SPM method, atomic force microscopy (AFM) has been widely used for cell topography imaging. However, soft cells are often slightly deformed by the mechanical force during the AFM imaging because of the interaction between the AFM probe and the sample surface even in the tapping mode. Interested readers can refer to a previous report for a detailed comparison between the SICM and the AFM when imaging fibroblast or myoblast cells. ${ }^{40}$ The SICM showed comparable resolution as the AFM, but no elastic deformation of cells was found in the topography images. SICM is an open system and can be integrated with a range of other techniques, including patch clamping, ${ }^{43}$ scanning electrochemical microscopy (SECM) ${ }^{44}$ confocal microscopy, ${ }^{34}$ Raman spectroscopy, and scanning near-field optical microscopy (SNOM). ${ }^{45}$ The auxiliary measurements can enable the quantitative measurements of various cellular parameters such as single ionchannel currents, membrane potentials, the flux of electroactive small molecules transported in and out of the cells, and cell membrane permeability. One of the major goals of this dissertation is to integrate the commercially available SICM set-up to study the morphologies and the extracellular membrane potential of the single living cells at the same time. 


\section{3: Different Aspect of Live Cell Imaging With SICM}

In this section, recent developments in operation and instrumentation of SICM and some of the most notable studies with this technique are introduced.

\subsection{1: Topography Imaging of Nanoparticles-Living Cell Membrane}

A thorough understanding of the interactions between natural and engineered nanoparticles (NPs) and cell surface is essential for the development of the fundamental knowledge of cell biology, the drug and gene delivery methods and the awareness of possible toxicity of engineered nanoparticles. Transmission electron microscopes (TEMs) can provide very high-resolution images of both NPs and cells. ${ }^{10}$ However, it is not possible to study the dynamic process of the NP-cell membrane interactions in native conditions. Fluorescence microscopy is the main method to study the effect of NPs to living cells. However, it is challenging to reveal structural changes with high resolution on the cell membrane. Also, the NPs need to be labeled, and this can be undesirable sometimes. As a label-free method, AFM is often used. However, AFM also has the drawbacks as I discussed earlier.

SICM has been successfully used to take high-resolution topography images of the

living cell membranes in their native environment. Several groups have studied ${ }^{23,} 46-51$ various types of living cell and the dynamics of membrane features. ${ }^{21,46-48}$ Besides the study of the cell membrane features, pioneering studies of the dynamic interactions between NPs and the living cell membranes have been carried out. Korchev and collaborators did a series of experiments to study the endocytosis of single polyoma viruslike particles (VLPs) on the living COS7 cell surface with the help of SICM. The cell surface was treated with the fluorescently labeled VLPs and studied using scanning surface 
confocal microscopy (SSCM) technique to track the interaction of VLPs with the cell membrane. The SSCM technique is a combination of SICM and confocal microscopy. ${ }^{49}$ By acquiring concurrent SICM topography and fluorescence microscope images, they correlate the corresponding topographical features with the fluorescence images and unambiguously differentiate VLPs from other submicron features of the cell surface using high-resolution SSCM. ${ }^{34}$ They successfully observed the attachment and distribution of VLPs in the cell membrane. The same group then did successive studies to understand the molecular nature of endocytic pits in the apical membrane of both fixed and living cells, which are clathrin-green fluorescent protein (GFP) transfected COS7 cells. ${ }^{50}$ They were not only able to observe the geometric structures of the pits, but also to determine their type. Among the identified pits, $89 \%$ were clathrin-coated pits (CCPs). They further investigated the dynamics of these clathrin coated pits. ${ }^{51}$ Interestingly, about $70 \%$ of pits were disappeared after covering by the protrusion grew from one side of the pit. Those protrusions and pits disappeared together with pit-associated clathrin-enhanced GFP (EGFP) and actin-binding protein-EGFP (Abp1-EGFP), ${ }^{51}$ as suggested by the fluorescence images.

Recently, Korchev and his coworkers have demonstrated the capability of the SICM to follow the processes of single nanoparticle interactions with the living cell membrane. ${ }^{25}$ They used both conventional hopping mode SICM and confocal fluorescence microscopy to image interactions between a single NP (about 200nm carboxyl-modified particles (CMPs)) and the membrane of human alveolar epithelial type 1-like (AT1) cells. Obvious membrane protrusions were clearly observed during the internalization of the CMPs. But they found that some dynamics of membrane processes were clearly faster than 
the frame rate of conventional hopping mode SICM set-up. To address the fast dynamics of cell membrane activities, they developed a fast SICM using fast shear piezo-actuator. They were able to track the dynamic interactions between a single CMP and AT1-like cells at a rate $15 \mathrm{~s} /$ frame using both the fast SICM technique and confocal fluorescence microscopy. The fast SICM technique is advantageous to reveal in real time not only the virus entry pathway but also the corresponding cell morphology changes during the endocytosis of nanoparticles. Also, this imaging technique can assess targeting ability of functionalized nanoparticles designed for drug delivery.

\subsection{2: Electrochemical Imaging of Living Cell Membrane}

Electrochemical imaging of the living cell membranes has received a great deal of attention because its potential to reveal their microscopic structural and physiological properties. ${ }^{52-58}$ Scanning electrochemical microscopy (SECM) has been developed to investigate localized electrochemical reactions and reveal the spatial distribution of electrochemical activities of a sample surface immersed in an electrolyte containing redox molecules. ${ }^{59-64}$ In recent years, SECM has been applied to the living cell analysis and imaging and showed success in understanding cell activities (such as cellular respiration and signal transduction) by monitoring the release or consumption of small molecules in the vicinity of the cell membrane. ${ }^{65}$ SECM uses a partially insulated ultramicroelectrode (UME) or nanoelectrode as the probe and redox current as the feedback signal. When the electrode approaches near the sample surface, the redox current on the probe tip changes to the electrochemical properties of the sample surfaces. When the UME raster scans above the sample surface, the electrochemical activities of the sample surface can be mapped. The surface electrochemical recognition capability of SECM is attractive for living cell 
imaging application. However, the position feedback mechanism of SECM is often not enough to achieve sub-micron spatial resolution, especially for the complicated cell membrane. In contrast, SICM has proven especially beneficial for imaging various cell surfaces with very high spatial resolution. However, SICM lacks the chemical recognition capabilities. Therefore, SICM images often need to be complemented with fluorescence images to interpret the data. Because of the similarities between SICM and SECM, researchers have developed hybrid SECM/SICM technique to utilize the advantages and overcome the drawbacks of both techniques. ${ }^{44,66,67}$ A representative set up of SECM/SICM is shown in Figure 1.2a. This hybrid technique provides robust height control and enables higher resolution electrochemical imaging of living cell membranes.

Multifunctional probe with both nanopipette and nanoelectrode components is needed for hybrid SECM/SICM technique. Several methods have been developed and reported previously. The fabricated probes can be roughly divided into two types. The first type of probe can be fabricated by depositing a conductive layer (i.e., gold or platinum (Pt)) on the outside wall of a single barrel nanopipette (Figure 1.2b). The electrode shape can be either ring structure ${ }^{44}$ or stripe structure. ${ }^{68}$ The conductive layer will be further coated with a Parylene ${ }^{69}$ or electrophoretic paint ${ }^{44,70}$ or atomic layer deposition (ALD) method deposited alumina oxide layer for insulating. The insulating layer can also improve the mechanical, chemical and electrochemical stability of the probe. Focused ion beam (FIB) can also be used to precisely expose the electrode surface if necessary. However, the throughput will be low, and the cost will be high if using FIB. Using the first type of probe, Hersam and coworkers demonstrated simultaneous topography and electrochemical imaging and which resolve $180 \mathrm{~nm}$ size gold patterns on a flat substrate. ${ }^{71}$ Matsue and 
Korchev's groups were able to differentiate horse radish peroxidase (HRP) and glucose oxidase (GOD) spotted enzyme patterns on the glass substrate with submicrometer resolution. ${ }^{44}$ The second type is based on dual barrel nanopipettes made from theta micropipettes (see the probe in Figure 5.1a). ${ }^{72}$ One pore can be filled with pyrolytic carbon ${ }^{73}$ or gold or platinum by electroless plating. ${ }^{74}$ The pyrolytic carbon electrode can be further modified with gold or Pt NPs if needed. The other pore will remain open for ionic current measurement in SICM mode. The fabrication of the second type of electrode is easier than the first type. Korchev and collaborators developed and tested the type of probe (named double-barrier carbon nanoprobe, DBCNP). The DBCNPs can reveal about $200 \mathrm{~nm}$ pores in polyethylene terephthalate (PET) membranes in both topography and electrochemical images. Several research groups are continuing to develop facial, quick and cost-effective methods to make reproducible and reliable probes which can be applicable for SICM/SECM. ${ }^{75-78}$ 

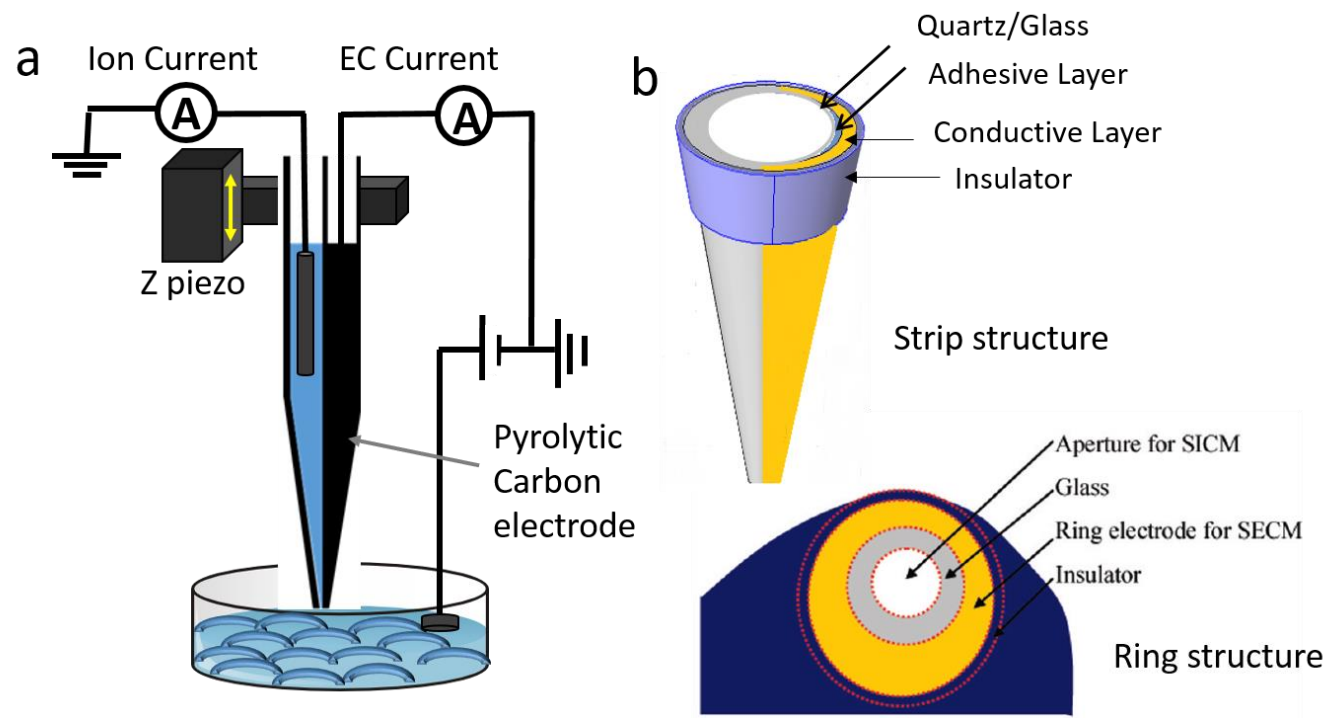

Figure1.2: SECM/SICM hybrid technique. (a) The schematic of a typical SECM/SICM setup using a double-barrier carbon nanoprobe (DBCNP). (b) The Schematic of type one SECM/SICM probes with stripe structure (top) and ring structure (bottom) metal electrode respectively. The ring structure figure is reprinted with permission from ref 34 (copyright 2010, ACS Publishing Group).

The SICM/SECM technique has been successfully used in living cell imaging. Matsue and Korchev's groups were able to get the topography, and electrochemical images of the convoluted living cell surface like that of the superior cervical ganglion (SCG), A6 live cells, and cardiomyocytes with higher spatial resolution using hopping mode feedback mechanism of SICM. In the SCG cell image, varicosities which control the neuron transduction and axon formation were clearly visualized. In an A6 living cell image, tight junction area and ridge-like structures formed because of the microvilli were revealed. Furthermore, they were able to characterize the permeation property of living cell membranes by monitoring the Faradic current of electroactive substances such as $\mathrm{O}_{2}$, $\mathrm{K}_{4}\left[\mathrm{Fe}(\mathrm{CN})_{6}\right]$, and $\mathrm{FcCH}_{2} \mathrm{OH}$ on cardiomyocytes. Korchev and collaborators demonstrated the capability of the DBCNPs by simultaneously recording topography and potential images of the living-sensory neurons and differentiated P12 cells. ${ }^{73}$ They also 
demonstrated the capability for localized $\mathrm{K}^{+}$delivery and simultaneous neurotransmitter detection from P12 cells. Although the electrochemical imaging by SECM/SICM with the DBCNPs provides high spatial resolution, the chemical sensitivity is reduced or lost. Paolo et al. demonstrated platinum coated carbon nanoelectrode could significantly enhance its electrochemical recognition function in the single cell analysis. ${ }^{79}$ Mustafa and coworkers have fabricated new platinum-based double barrel probes to enhance the electrochemical signal of SECM/SICM. ${ }^{80}$ They deposited platinum electrochemically over the carbon side of the DBCNPs which amplifies the electrochemical response of the nanoelectrode because of the enlarged total flux of the electroactive species at the electrode surface. They used the Pt deposited DBCNP probe for electrochemical imaging of immunocytochemically stained EGFR proteins in A431 cells and found increased sensitivity and resolution for the electrochemical images.

\section{4: Extracellular Potential Mapping of Living Cell Membrane}

Besides the topographical and electrochemical imaging, electrical potential or surface charge measurement and mapping are another important progress of SICM. Inspired by the voltage scan technique developed by Fromter in $1972,{ }^{81}$ and the study of trans and paracellular conductivity of flat epithelial cells by Formm and coworkers with the technique, ${ }^{82-88}$ researchers are striving to use the robust feedback mechanism of the SICM to study local potential distributions of porous membranes with high spatial resolution. By using the modified SICM technique, it is possible to position the probe very close to the living cell membrane and record submicron resolution extracellular potential distributions near the living cell membrane along with its topography image. 
Recently the Baker's group has developed potentiometric SICM (P-SICM) to record both ionic current and potential of a sample surface immersed in the electrolyte solution. ${ }^{89-91}$ The P-SICM uses the double barrel quartz theta nanopipettes. Ag/AgCl electrodes are inserted into both electrolyte-filled barrels. One barrel is used for the position control of the nanopipette by sensing the ionic current. The other barrel is used for the potential measurement on the reference electrode immersed in the bath solution. Baker's group did a series of experiments on the porous silicon nitride ( $\mathrm{SiN})$ membrane using both small (60 $\mathrm{nm}$ diameter) and large (175 $\mathrm{nm}$ diameter) theta nanopipettes to demonstrate the capability of the P-SICM quantitatively. The geometries of the nanopores in the $\mathrm{SiN}$ membrane were well-calibrated by scanning electron microscopy (SEM). They were able to take potential images of the nanopores in the $\mathrm{SiN}$ membrane, and the $68 \mathrm{~nm}$ diameter nanopore in the SiN membrane can still be recognized. They also simultaneously recorded ionic current and potential across a particular nanopore during a single line scan. As expected, the pore geometry was better resolved with the small diameter nanopipette. Also, the measured nanopore diameter of the potential image is bigger than the actual nanopore diameter, and the discrepancy is bigger for large nanopipette. The bigger pore diameter in the potential image is attributed to the enlarged electric field distribution region beyond the nanopore periphery and the bigger probe-sample distance for large nanopipette during imaging. They also confirmed the potential electrode of the theta pipette measures the potential at the pipette tip, and the potential drop at the tip is because of the increased access resistance when probe-sample distance decreases. Therefore the signal-to-noise ratio (SNR) of potential measurement can be improved by using a smaller diameter nanopipette. 
However, we need to pay the price for the increased SNR of ionic current for small nanopipette.

Baker's group has also used the P-SICM technique to study living cells. They distinguished transcellular and paracellular conductive pathway in epithelial cells and reported that the SNR for potentiometric measurement was better than the ionic current measurement. ${ }^{89}$ Although the potential and the ionic current can be measured at the same time at selected positions of the cell membrane, simultaneous recording topography and potential images of the whole cell have not been reported yet. It is one of my major research project for my dissertation.

\section{5: Overview of the Research Projects and Results}

The ultimate goal of my dissertation is to develop the multifunctional SICM techniques for single cell studies. To achieve my goal, in the beginning, morphology change of fixed HeLa cells were studied during the endocytosis of the conjugated polymer nanoparticles (CPNs) with SICM. Only the topography images were recorded and analyzed in the first project. After familiar of the SICM imaging technique, commercially available SICM was integrated to record the both topography and potential images of the living cells. For the potential imaging required hardware and software were developed. Double barrel theta pipette was used to map the topography and the extracellular potential distribution of the living melanoma and melanocytes. However, the sensitivity of the potential measurement was pretty low only with bare theta pipette. The multifunctional nanopipette was developed and used to increase the sensitivity of the potential measurement.

The high quality probe is always very important for all types of SPM techniques. So, multifunctional nanopipettes were fabricated and characterized by using scanning 
electron microscopy (SEM), ionic current measurement and cyclic voltammetry measurement. The pyrolytic carbon deposited one barrel of the theta nanopipette and the other barrel was remain open. The theta nanopore/carbon electrode was used to demonstrate its capability for simultaneous detection of ionic current and local electrical potential changes during translocation of $40 \mathrm{~nm}$ sized, charged gold nanoparticles (GNPs) through the nanopore. The observed experimental result successfully demonstrated local potential change during translocation of a single GNP with perfect correlation with current change as well as collective potential change because of a cluster of GNPs outside the nanopore entrance. The sensitivity of the potential measurement was even better than the sensitivity of the ionic current measurement.

Finite element numerical simulations were also performed to understand the fundamental charge sensing mechanism during the translocation of the $40 \mathrm{~nm}$ charged GNPs through the nanopore/ nanoelectrode using similar conditions as we used in our experiment. The simulation results were similar to the experimental results. Altogether, these results suggest that multifunctional nanopipette is a new nanopore type biosensor for DNA, protein, nanoparticle and virus analysis with various size and charge. The multifunctional nanopipette can also directly used as a probe for the hybrid SICM/SECM technique.

Moreover, my dissertation presents the study of size and shape of the conjugated polymer nanoparticles (CPNs) under complexation of Hyaluronic Acid with the Atomic Force Microscopy (AFM) techniques. Core-Shell nanoparticles via controlled aggregation of semiflexible conjugated polymer and hyaluronic acid were synthesized for the targeted drug and gene delivery.Size and shape of these polymer nanoparticles play a very critical 
role in drug delivery. So to confirm its size and shape of these core-shell nanoparticles, AFM was used to image these nanoparticles with tapping mode in air. Both topography and phase images clearly demonstrated donut shaped core-shell nanoparticles with later size about $58 \pm 13 \mathrm{~nm}$ which was pretty similar to the hydrodynamic radius measured by the dynamic light scattering technique.

\section{6: References}

1. Altschuler, S.J. \& Wu, L.F. Cell 2010, 141, 559.

2. Adams, D. \& Levin, M. Cell and Tissue Research 2012, 1.

3. Miesenbock, G., De Angelis, D.A. \& Rothman, J.E. Nature 1998, 394, 192.

4. Bradley, J., Luo, R., Otis, T.S. \& DiGregorio, D.A. J Neurosci 2009, 29, 9197.

5. Chernet, B.T. \& Levin, M. Disease Models \& Mechanisms 2013, 6, 595.

6. Adams, D.S. \& Levin, M. Cold Spring Harbor Protocols 2012, 2012, pdb.top067710.

7. Jagtap, R. \& Ambre, A. 2006.

8. Weisenhorn, A.L., Khorsandi, M., Kasas, S., Gotzos, V. \& Butt, H.J. Nanotechnology 1993, 4, 106.

9. Burnham, N.A. \& Colton, R.J. Journal of Vacuum Science \& Technology A: Vacuum, Surfaces, and Films 1989, 7, 2906.

10. Verma, A. \& Stellacci, F. Small 2010, 6, 12.

11. Zhong, Q., Inniss, D., Kjoller, K. \& Elings, V.B. Surface Science 1993, 290, L688.

12. Lee, G.U., Kidwell, D.A. \& Colton, R.J. Langmuir 1994, 10, 354.

13. Hugel, T. \& Seitz, M. Macromolecular rapid communications 2001, 22, 989.

14. Lee, G.U., Chrisey, L.A. \& Colton, R.J. SCIENCE-NEW YORK THEN WASHINGTON- 1994, 771. 
15. Oesterhelt, F., Rief, M. \& Gaub, H. New Journal of Physics 1999, 1, 6.

16. Knowles, T.P., Fitzpatrick, A.W., Meehan, S., Mott, H.R., Vendruscolo, M., Dobson, C.M. \& Welland, M.E. Science 2007, 318, 1900.

17. Binnig, G., Rohrer, H., Gerber, C. \& Weibel, E. Physical Review Letters 1982, 49, 57.

18. Binnig, G. \& Rohrer, H. Surface Science 1983, 126, 236.

19. Binning, G. Review of Modern Physics 1987, 59, 615.

20. Binnig, G., Quate, C. \& Gerber, C. Phys Rev Lett 1986, 56, 930

21. Anariba, F., Anh, J.H., Jung, G.-E., Cho, N.-J. \& Cho, S.-J. Modern Physics Letters B 2012, 26, 1130003.

22. Hansma, P., Drake, B., Marti, O., Gould, S. \& Prater, C. Science 1989, 243, 641.

23. Korchev, Y., Bashford, C., Milovanovic, M., Vodyanoy, I. \& Lab, M. Biophys J 1997, 73, 653

24. Gu, Y., Gorelik, J., Spohr, H.A., Shevchuk, A., Harding, S.E., Vodyanoy, I., Klenerman, D. \& Korchev, Y.E. The FASEB Journal 2002, 16, 748.

25. Novak, P., Shevchuk, A., Ruenraroengsak, P., Miragoli, M., Thorley, A.J., Klenerman, D., Lab, M.J., Tetley, T.D., Gorelik, J. \& Korchev, Y.E. Nano letters 2014, 14, 1202.

26. Robertson, G.A. Circulation research 2013, 112, 1088.

27. Lazenby, R.A., McKelvey, K. \& Unwin, P.R. Analytical Chemistry 2013, 85, 2937.

28. Shen, M., Ishimatsu, R., Kim, J. \& Amemiya, S. Journal of the American Chemical Society 2012, 134, 9856.

29. Chen, C.-C., Zhou, Y. \& Baker, L.A. Annual Review of Analytical Chemistry 2012, 5, 207.

30. Hansma, P., Drake, B., Marti, O., Gould, S. \& Prater, C. Science 1989, 243, 641

31. Pastré, D., Iwamoto, H., Liu, J., Szabo, G. \& Shao, Z. Ultramicroscopy 2001, 90, 13. 
32. Novak, P., Li, C., Shevchuk, A., Stepanyan, R., Caldwell, M., Hughes, S., Smart, T., Gorelik, J., Ostanin, V., Lab, M., Moss, G., Frolenkov, G., Klenerman, D. \& Korchev, Y. Nat Methods 2009, 6, 279

33. Happel, P. \& Dietzel, I. Journal of Nanobiotechnology 2009, 7, 7.

34. Shevchuk, A.I., Hobson, P., Lab, M.J., Klenerman, D., Krauzewicz, N. \& Korchev, Y.E. Biophysical journal 2008, 94, 4089.

35. Shin, W. \& Gillis, K.D. Biophysical journal 2006, 91, L63.

36. Gorelik, J., Shevchuk, A.I., Frolenkov, G.I., Diakonov, I.A., Lab, M.J., Kros, C.J., Richardson, G.P., Vodyanoy, I., Edwards, C.R.W., Klenerman, D. \& Korchev, Y.E. Proceedings of the National Academy of Sciences 2003, 100, 5819.

37. Gorelik, J., Zhang, Y., Shevchuk, A.I., Frolenkov, G.I., Sánchez, D., Lab, M.J., Vodyanoy, I., Edwards, C.R.W., Klenerman, D. \& Korchev, Y.E. Molecular and Cellular Endocrinology 2004, 217, 101.

38. Ying, L., Bruckbauer, A., Zhou, D., Gorelik, J., Shevchuk, A., Lab, M., Korchev, Y. \& Klenerman, D. Physical Chemistry Chemical Physics 2005, 7, 2859.

39. Shevchuk, A.I., Frolenkov, G.I., Sánchez, D., James, P.S., Freedman, N., Lab, M.J., Jones, R., Klenerman, D. \& Korchev, Y.E. Angewandte Chemie International Edition 2006, 45, 2212.

40. Rheinlaender, J., Geisse, N.A., Proksch, R. \& Schäffer, T.E. Langmuir 2010, 27, 697.

41. Miragoli, M., Moshkov, A., Novak, P., Shevchuk, A., Nikolaev, V.O., ElHamamsy, I., Potter, C.M.F., Wright, P., Kadir, S.H.S.A., Lyon, A.R., Mitchell, J.A., Chester, A.H., Klenerman, D., Lab, M.J., Korchev, Y.E., Harding, S.E. \& Gorelik, J. Journal of The Royal Society Interface 2011, 8, 913.

42. Korchev, Y.E., Gorelik, J., Lab, M.J., Sviderskaya, E.V., Johnston, C.L., Coombes, C.R., Vodyanoy, I. \& Edwards, C.R. Biophysical journal 2000, 78, 451.

43. Korchev, Y.E., Negulyaev, Y.A., Edwards, C.R.W., Vodyanoy, I. \& Lab, M.J. Nat Cell Biol 2000, 2, 616.

44. Takahashi, Y., Shevchuk, A.I., Novak, P., Murakami, Y., Shiku, H., Korchev, Y.E. \& Matsue, T. Journal of the American Chemical Society 2010, 132, 10118.

45. Rothery, A.M., Gorelik, J., Bruckbauer, A., Yu, W., Korchev, Y.E. \& Klenerman, D. Journal of Microscopy 2003, 209, 94. 
46. Shevchuk, A.I., Frolenkov, G.I., Sánchez, D., James, P.S., Freedman, N., Lab, M.J., Jones, R., Klenerman, D. \& Korchev, Y.E. Angewandte Chemie 2006, 118, 2270.

47. Gorelik, J., Shevchuk, A.I., Frolenkov, G.I., Diakonov, I.A., Lab, M.J., Kros, C.J., Richardson, G.P., Vodyanoy, I., Edwards, C.R., Klenerman, D. \& Korchev, Y.E. Proceedings of the National Academy of Sciences of the United States of America 2003, 100, 5819.

48. Shevchuk, A.I., Gorelik, J., Harding, S.E., Lab, M.J., Klenerman, D. \& Korchev, Y.E. Biophysical journal 2001, 81, 1759.

49. Gorelik, J., Shevchuk, A., Ramalho, M., Elliott, M., Lei, C., Higgins, C., Klenerman, D., Krauzewicz, N. \& Korchev, Y. Proceedings of the National Academy of Sciences 2002, 99, 16018.

50. Shevchuk, A.I., Hobson, P., Lab, M.J., Klenerman, D., Krauzewicz, N. \& Korchev, Y.E. Pflugers Archiv : European journal of physiology 2008, 456, 227.

51. Shevchuk, A.I., Novak, P., Taylor, M., Diakonov, I.A., Ziyadeh-Isleem, A., Bitoun, M., Guicheney, P., Lab, M.J., Gorelik, J., Merrifield, C.J., Klenerman, D. \& Korchev, Y.E. The Journal of cell biology 2012, 197, 499.

52. Yasukawa, T., Kaya, T. \& Matsue, T. Electroanalysis 2000, 12, 653.

53. Liu, B., Cheng, W., Rotenberg, S.A. \& Mirkin, M.V. Journal of Electroanalytical Chemistry 2001, 500, 590.

54. Cai, C., Liu, B., Mirkin, M.V., Frank, H.A. \& Rusling, J.F. Analytical chemistry 2002, 74, 114.

55. Kaya, T., Torisawa, Y.-s., Oyamatsu, D., Nishizawa, M. \& Matsue, T. Biosensors and Bioelectronics 2003, 18, 1379.

56. Takii, Y., Takoh, K., Nishizawa, M. \& Matsue, T. Electrochimica Acta 2003, 48, 3381.

57. Feng, W., Rotenberg, S.A. \& Mirkin, M.V. Analytical chemistry 2003, 75, 4148.

58. Bard, A.J., Li, X. \& Zhan, W. Biosensors \& bioelectronics 2006, 22, 461.

59. Beaulieu, I., Kuss, S., Mauzeroll, J. \& Geissler, M. Analytical chemistry 2011, 83, 1485.

60. Li, X. \& Bard, A.J. Journal of Electroanalytical Chemistry 2009, 628, 35. 
61. Zhu, L., Gao, N., Zhang, X. \& Jin, W. Talanta 2008, 77, 804.

62. Edwards, M.A., Martin, S., Whitworth, A.L., Macpherson, J.V. \& Unwin, P.R. Physiological measurement 2006, 27, R63.

63. Liu, B., Rotenberg, S.A. \& Mirkin, M.V. Analytical chemistry 2002, 74, 6340.

64. Liu, B., Rotenberg, S.A. \& Mirkin, M.V. Proceedings of the National Academy of Sciences 2000, 97, 9855 .

65. Beaulieu, I., Kuss, S., Mauzeroll, J. \& Geissler, M. Analytical Chemistry 2011, 83, 1485.

66. Takahashi, Y., Shevchuk, A.I., Novak, P., Babakinejad, B., Macpherson, J., Unwin, P.R., Shiku, H., Gorelik, J., Klenerman, D., Korchev, Y.E. \& Matsue, T. Proceedings of the National Academy of Sciences 2012, 109, 11540.

67. Zhou, L., Zhou, Y. \& Baker, L.A. Electrochemical Society Interface 2014, 47.

68. Morris, C.A., Chen, C.-C. \& Baker, L.A. Analyst 2012, 137, 2933.

69. Derylo, M.A., Morton, K.C. \& Baker, L.A. Langmuir 2011, 27, 13925.

70. Walsh, D.A., Fernández, J.L., Mauzeroll, J. \& Bard, A.J. Analytical Chemistry 2005, 77, 5182.

71. Comstock, D.J., Elam, J.W., Pellin, M.J. \& Hersam, M.C. Analytical Chemistry 2010, 82, 1270.

72. Lai, S.C.S., Dudin, P.V., Macpherson, J.V. \& Unwin, P.R. Journal of the American Chemical Society 2011, 133, 10744.

73. Takahashi, Y., Shevchuk, A.I., Novak, P., Zhang, Y., Ebejer, N., Macpherson, J.V., Unwin, P.R., Pollard, A.J., Roy, D. \& Clifford, C.A. Angewandte Chemie International Edition 2011, 50, 9638.

74. Menon, V.P. \& Martin, C.R. Analytical Chemistry 1995, 67, 1920.

75. Thakar, R., Weber, A.E., Morris, C.A. \& Baker, L.A. The Analyst 2013, 138, 5973.

76. Takahashi, Y., Shevchuk, A.I., Novak, P., Zhang, Y., Ebejer, N., Macpherson, J.V., Unwin, P.R., Pollard, A.J., Roy, D., Clifford, C.A., Shiku, H., Matsue, T., Klenerman, D. \& Korchev, Y.E. Angew Chem Int Ed Engl 2011, 50, 9638. 
77. Nadappuram, B.P., McKelvey, K., Al Botros, R., Colburn, A.W. \& Unwin, P.R. Analytical chemistry 2013, 85, 8070.

78. Comstock, D.J., Elam, J.W., Pellin, M.J. \& Hersam, M.C. Analytical chemistry $2010,82,1270$.

79. Actis, P., Tokar, S., Clausmeyer, J., Babakinejad, B., Mikhaleva, S., Cornut, R., Takahashi, Y., López Córdoba, A., Novak, P. \& Shevchuck, A.I. ACS nano 2014, 8,875 .

80. Sen, M., Takahashi, Y., Matsumae, Y., Horiguchi, Y., Kumatani, A., Ino, K., Shiku, H. \& Matsue, T. Analytical chemistry 2015.

81. Frömter, E. The Journal of membrane biology 1972, 8, 259.

82. Gitter, A., Bertog, M., Schulzke, J.-D. \& Fromm, M. Pflügers Archiv 1997, 434, 830.

83. Bendfeldt, K., Gitter, A.H. \& Fromm, M. Annals of the New York Academy of Sciences 1998, 859, 295.

84. Gitter, A.H., Bendfeldt, K., Schulzke, J.-D. \& Fromm, M. The Faseb Journal 2000, $14,1749$.

85. Bojarski, C., Gitter, A., Bendfeldt, K., Mankertz, J., Schmitz, H., Wagner, S., Fromm, M. \& Schulzke, J. The Journal of physiology 2001, 535, 541.

86. Florian, P., Schöneberg, T., Schulzke, J.D., Fromm, M. \& Gitter, A.H. The Journal of Physiology 2002, 545, 485.

87. Heller, F., Fromm, A., Gitter, A.H., Mankertz, J. \& Schulzke, J.D. Mucosal immunology 2008, 1 Suppl 1, S58.

88. Yu, A.S., Cheng, M.H., Angelow, S., Gunzel, D., Kanzawa, S.A., Schneeberger, E.E., Fromm, M. \& Coalson, R.D. The Journal of general physiology 2009, 133, 111.

89. Chen, C.C., Zhou, Y., Morris, C.A., Hou, J. \& Baker, L.A. Analytical chemistry 2013, 85, 3621 .

90. Zhou, Y., Chen, C.C., Weber, A.E., Zhou, L. \& Baker, L.A. Langmuir 2014, 30, 5669 .

91. Zhou, Y., Chen, C.-C., Weber, A.E., Zhou, L., Baker, L.A. \& Hou, J. Tissue barriers 2013, 1 . 


\section{CHAPTER 2: METHODS AND MATERIALS}

This dissertation used three imaging techniques, Atomic Force Microscopy (AFM), Scanning Ion Conductance Microscopy (SICM), and Fluorescence Microscopy to study and analyze single cells, single nanoparticles, and the interaction between the cell membrane and nanoparticles during endocytosis. It also provides the details of the multifunctional probe fabrication and its characterization. In overall, this chapter presents all the methods, experimental and theoretical, which I used to complete my dissertation research.

2.1: Characterization of Conjugated Polymer Nanoparticles (CPNs) With Atomic Force Microscopy

Atomic force microscopy (AFM) is a powerful tool to characterize the size and shape of the polymer nanoparticles. It can generate the three-dimensional topographic images of the sample features with sub-nanometer resolution. Tapping mode atomic force microscopy was used to image the conjugated polymer nanoparticles (CPNs). Phase images were recorded simultaneously with topography images. The sample was prepared on a mica surface. The component (3-aminopropyl) triethoxysilane (APTES) was vapor deposited on a freshly cleaved mica surface for $80 \mathrm{~min}$ in a desiccator filled with dry Argon. $30 \mu \mathrm{L}$ of $\mathrm{CP} / \mathrm{HA}$ complex in deionized (DI) water was placed on the APTES coated mica and incubated for 30-45 min in a laminar flow hood. The droplet was then rinsed away with $\sim 1 \mathrm{~mL}$ DI water (18 M ohm) and dried gently with dry argon. Images were acquired with a MultiMode5 AFM microscope (Bruker, Santa Barbara, CA) operated in tapping mode in air using a 1.58-1.62 V oscillation amplitude with uncoated silicon AFM tips (T190, vistaprobes, $\mathrm{k} \sim 40 \mathrm{~N} / \mathrm{m}$ ) at a resonance frequency of $190 \mathrm{kHz}$. Typically, areas of 
$1 \times 1 \mu \mathrm{m} 2$ were scanned at a rate of $0.5-1 \mathrm{~Hz}$ and a resolution of $512 \times 512$ pixels. All the experiments were performed at room temperature. The images were further processed by Image Analysis Software Gwyddion.

\section{2: Scanning Ion Conductance Microscopy (SICM) for Single Cell Membrane Imaging}

A commercial SICM (XE-Bio, Park Systems) was used to study the change in morphology of the fixed HeLa cell as well as living HeLa cell membranes during endocytosis of polymer nanoparticles by recording the topography images. The SICM was housed on an inverted optical microscope (Nikon Eclipse Ti-U) to locate the nanopipette tip on the position of interest, and the optical images were captured by a CCD camera (UEye). The whole system was placed on an air-floated optical table to reduce mechanical noise. Approach-Retract Scanning (ARS) mode (or so-called hopping mode) ${ }^{1}$ was used to control nanopipette position during SICM imaging. The scheme of the SICM setup is shown in Figure 1.1a. An optical microscope image showing both the cells and the pipette tip is shown in Figure 2.1. A typical approaching curve (current vs. distance) is shown in Figure 1.1b. The curve can be fitted by equation 2.1 (see in the section 2.2.2.2). The set point ionic current $I_{\mathrm{sp}}$ (indicated by the red dash line) for imaging is $98 \%$ of the initial current $\mathrm{I}_{0}$.

The time to acquire a $256 \times 256$ pixel SICM image was typically $40-50 \mathrm{~min}$. The instrument lateral $(\mathrm{x}-\mathrm{y})$ resolution was about $50 \mathrm{~nm}$, which was close to the nanopipette inner diameter. ${ }^{2}$ The typical image resolution was about $100-150 \mathrm{~nm} /$ pixel. The vertical (z) direction resolution was about $10 \mathrm{~nm}$. It should be noted that SICM can measure the height of soft objects with little mechanical compression. The $\mathrm{x}-\mathrm{y}$ and $\mathrm{z}$ resolution were confirmed 
by using soft PDMS microstructures and single and double membrane layers of broken red blood cells. ${ }^{3}$

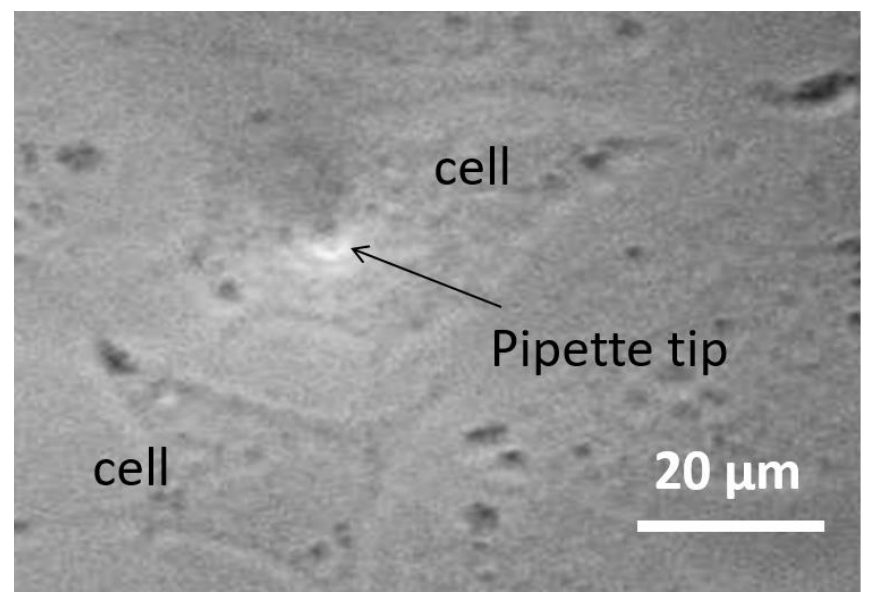

Figure 2.1: The optical image of nanopipette located above living HeLa cell monolayer before performing scanning (scale bar $20 \mu \mathrm{m}$ ), the arrowhead points the location of nanopipette, which is much larger than the real size of the nanopipette tip.

\subsection{1: Nanopipette Fabrication}

The SICM uses the nanopipette as a probe. For only the topography images, single barrel borosilicate glass nanopipette was used, while for the simultaneous imaging of the potential and topography features double barrel quartz theta nanopipette was used.

\subsubsection{1: Single Barrel Glass Nanopipette}

The borosilicate glass capillary tubes with filament and fire polished (BF100-5815), Sutter Instrument) were first cut in the middle with a diamond pencil to make two equal length microcapillary tubes. The edge of the cutting side of those glass pipette was fire polished manually with the Bunsen burner. Those glass pipettes were cleaned by piranha (caution: Piranha solutions are highly corrosive and must be handled with extreme caution) for 30 minutes. The cleaned pipettes rinsed with deionized water, and then dried in an oven at $120^{\circ} \mathrm{C}$ for 15 minutes. Glass nanopipettes were fabricated from these cleaned 
capillary tubes by using a laser-based pipette puller (P-2000, Sutter Instrument) with the following parameters: $\mathrm{HEAT}=275, \mathrm{FIL}=4, \mathrm{VEL}=50, \mathrm{DEL}=225, \mathrm{PUL}=150$.

\subsubsection{2: Double barrel quartz theta nanopipette}

The quartz theta capillary tubes with filament (FG-G QT120-90-7.5, Sutter Instrument) were first cleaned by Piranha (caution: Piranha solutions are highly corrosive and need to be handled with extreme caution!) for 30 minutes and then repeatedly rinsed with deionized water and dried in an oven at $120{ }^{\circ} \mathrm{C}$ for overnight. Quartz dual nanopore nanopipettes were fabricated from these cleaned capillary tubes by using a laser pipette

puller (P-2000, Sutter Instrument) with following parameters: HEAT=825, FIL=3, VEL=40, DEL=220, PUL=190 for nanopore/carbon-nanoelectrode fabrication. Different parameters were used during the pulling to adjust the pore diameter according to the purpose of the experiment. For the living cell membrane extracellular potential measurement, following parameters were used HEAT=835, FIL=3, VEL=40, DEL=220, PUL=165 were used.

\subsection{2: Nanopipette Characterization}

Scanning electron microscopy (SEM), pore conductance measurement and optical microscope bright field imaging were carried out to characterize the nanopipette pore geometry.

\subsubsection{1: SEM}

Field emission scanning electron microscope (FE-SEM, JEOL JSM-6330F) was used to characterize the nanopipette geometry. The nanopipette was coated with about 5$6 \mathrm{~nm}$ thick gold using auto sputter coater (PELCOSC-7) to make the surface conducting 
and avoid the charging effect during SEM measurements. Figure 2.2a and 2.2b represent SEM characterization for the geometry of the single barrel glass nanopipette tip. SEM characterization of the double barrel theta nanopipette tip and size of the nanopore and carbon electrode of nanopore/CNE is shown in Figures 5.1 in chapter 5. The half cone angle of fabricated nanopipettes was estimated using ImageJ software. The half cone angle from the SEM images of the 5 representatives single barrel glass nanopipette and double barrel quartz theta nanopipette were estimated $2^{\circ}$ and $13^{\circ}$ respectively. The estimated half cone angle for nanopipette was used in the simulation geometry as well as for the estimation of nanopore diameter from IV measurement.
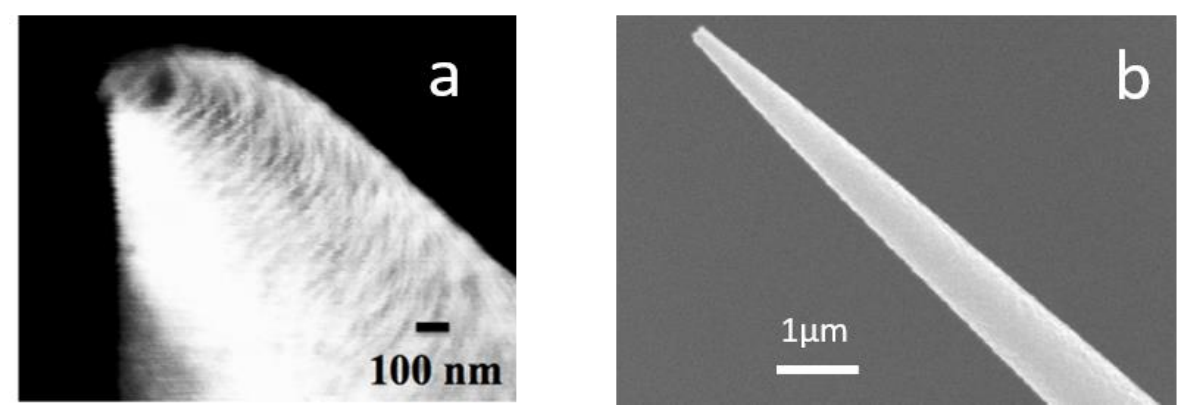

Figure 2.2: SEM image of a typical glass nanopipette used for SICM. (a) Top view of the nanopipette (scale bar $100 \mathrm{~nm}$ ).(b) Side view of the nanopipette. The half cone angle is about 2 degree from the SEM image. The nanopipette was coated with about 5-6 nm thick gold for SEM imaging.

\subsubsection{2: Measurement of pore diameter from the IV measurement}

The nanopipette was filled with the same electrolyte (1X PBS) as the bath solution and an $\mathrm{Ag} / \mathrm{AgCl}$ wire electrode (prepared by dipping clean $0.2 \mathrm{~mm}$ diameter $\mathrm{Ag}$ wires in bleach for 30 minutes) was inserted from the back. The I-V curves of the nanopipette were measured by a source measure unit 2636A (Keithley Instruments, Cleveland, Ohio). Bias was applied below $1 \mathrm{~V}$, generally $(-0.4$ to $+0.4 \mathrm{~V})$ with a scan rate of $50 \mathrm{mV} / \mathrm{s}$ to avoid 
hydrolysis and instability of reference electrodes. All measurements were performed at room temperature. The measurement setup was housed in a home-built Faraday cage to reduce external noise.

The inner diameter of the nanopipette was estimated based on current-voltage (IV) curves as shown in figure $2.3 \mathrm{a}$ and $2.3 \mathrm{~b}$. As demonstrated previously by both experiments and simulations, ${ }^{4-6}$ surface charge effect to the I-V curves at low bias range ($20 \mathrm{mV}$ to $+20 \mathrm{mV}$ ) was ignored, and nanopipette diameter was estimated by using a simple analytical equation. The following analytical equations 2.1 and 2.2 were used for the single barrel and double barrel nanopipette respectively.

$$
\begin{aligned}
& \mathrm{D}=\frac{2}{k R_{p}}\left(\frac{1}{\pi \tan \theta}\right) \\
& \mathrm{D}=\frac{4}{k R_{p}}\left(\frac{1}{\pi \tan \theta}\right)
\end{aligned}
$$

where $\kappa$ is the conductivity of the electrolyte. Nanopore resistance Rp is derived from the reciprocal of the slope of the linear portion of the I-V curve within a small applied bias range. $(-20 \mathrm{mV}$ to $+20 \mathrm{mV})$ as in Figure 2.3b. From the pore resistance $0.16 \mathrm{G} \Omega$ (Figure 2.3c) and half cone angle 2 , the calculated pore diameter $\mathrm{D}$ is about $74 \mathrm{~nm}$ using eq. 2.1. The calculated size is consistent with the SEM image. Similarly, theta pipette was also characterized by the IV measurement which has explained in chapter 5 under section (5.2.1). 

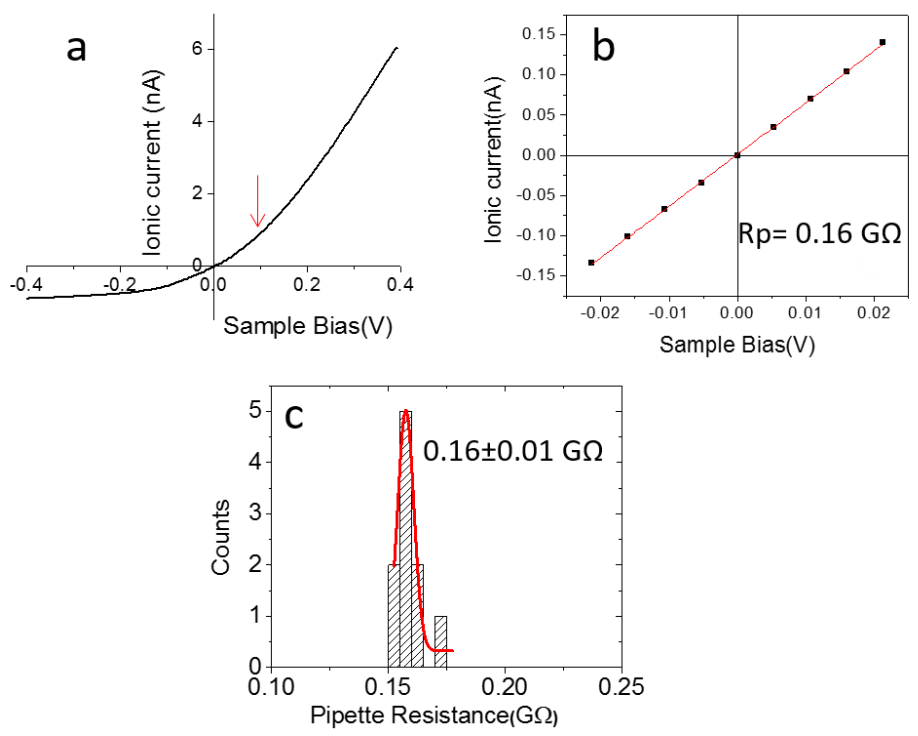

Figure 2.3. (a) The IV curve of a typical glass nanopipette in 1x PBS buffer before approaching the nanopipette to the substrate surface. The arrow indicates the current normally used in SICM experiment. (b) The IV curve at small bias range (-20 $\mathrm{mV}$ to +20 $\mathrm{mV}$ ). The red curve is the linear fitting to the experimental data (black dots), and the pore resistance $\mathrm{Rp}=0.16 \mathrm{G} \Omega$ was obtained from the slope of the fitting line. (c) The Histogram of the pore resistance $(\mathrm{Rp})$ of 10 nanopipettes. The red curve is the Gaussian fit, and the mean value is $0.16 \pm 0.01 \mathrm{G} \Omega$.

\subsubsection{3: Optical microscope bright field imaging}

An optical microscope (Nikon Eclipse Ti-U) with 40X lens was also used to characterize the nanopipettes. Figure $2.4 \mathrm{a}$ and $2.4 \mathrm{~b}$ represent the typical optical images of theta nanopipettes before and after carbon deposition.

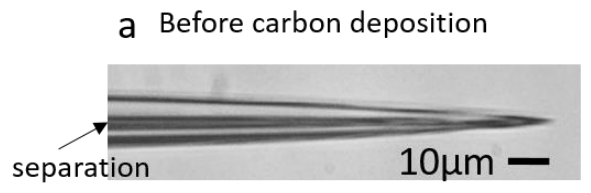

b After carbon deposition

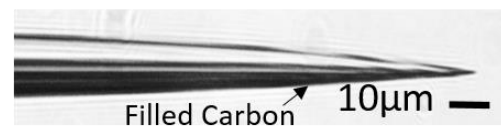

Figure 2.4 The optical images (a) a dual-nanopore nanopipette (before carbon deposition) where the black arrow indicates the separation between two barrels. (b) nanopore/CNE nanopipette (after carbon deposition) where the black arrow indicates the filled solid carbon only in one barrel. 


\subsection{3: Confocal Fluorescence Imaging}

A confocal microscope generates high-resolution images of a specimen as compared to the conventional fluorescence microscopy. It is designed to eliminate most of the light from the specimen that is out of the microscope's focal plane. When the laser focus is moved over the specimen, the entire image is generated by scanning a single point at a time in an X-Y manner. By scanning many thin sections from the sample, very clean and high-resolution three-dimensional image of the sample can be reconstructed. ${ }^{7}$ Fluorescence images of the living, or fixed Hela cells were obtained using a Nikon A1R confocal fluorescence microscope with 40x oil immersion lens at Prof. V. Moy's lab at the University of Miami. The fluorescence was excited by a $488 \mathrm{~nm}$ laser, and a band-pass filter $(500-550 \mathrm{~nm})$ was used for imaging CPNs. The HeLa cells $(\approx 50000$ per well) were seeded into a microscopy chamber ( $1 \mu$-Slide 8 -well ibiTreat, ibidi, Germany) with $0.5 \mathrm{~mL}$ of complete MEM medium. After $24 \mathrm{~h}$ of culture at $37{ }^{\circ} \mathrm{C}$, cells were treated with $10 \times 10$ $-6 \mathrm{M}$ of CPN for $1 \mathrm{~h}$ and $4 \mathrm{~h}$. After washing with $1 \times \mathrm{PBS}$ for three times, some cells were fixed with 4\% (w/v) paraformaldehyde

\subsection{4: Data Collection and Analysis}

The statistical analysis of data was carried out by XEI (Park Systems), Gwyddion ${ }^{8}$ and Origin (Origin Lab Corp.) software. Because of the large curvature of the cell surface, enhanced color topography images were used to reveal the small height changes of the cell membrane. For enhanced color image, the color of a pixel was determined by how much of a change it had compared to its neighbors. The height contrast of the image was therefore enhanced. The surface area ratio (similar to the roughness) of the cell membrane was calculated over the whole cell membrane, and the cell membrane was separated from the 
substrate by a height threshold method using Gwydion. The surface area ratio data from at least three cells of each batch was collected and repeated at least three batches to avoid cell to cell and batch to batch variations. All the data were combined to get statistical mean values and standard variations.

\subsection{5: Calculation of Surface Area Ratio}

The surface area ratio was calculated using Gwyddion software as follow. Suppose surface area as 1 by 1 pixel $(0.156 \mu \mathrm{m}$ by $0.156 \mu \mathrm{m}$ in current report $), 1$ by 1 pixel is an area composed of four different points, as shown in Figure 2.5, each point's value is named as $\mathrm{Z} 1, \mathrm{Z} 2, \mathrm{Z} 3$ and $\mathrm{Z} 4 . \mathrm{Z} 5$ is calculated as an average height value from $\mathrm{Z} 1$ to $\mathrm{Z} 4$, and located in the middle of them. Now, there is four surface (A1, A2, A3 and A4), which adds up to the area of 1 by 1 pixel. Geometric Area in Figure 2.5 is shown as the region in the X-Y panel below the gray area.

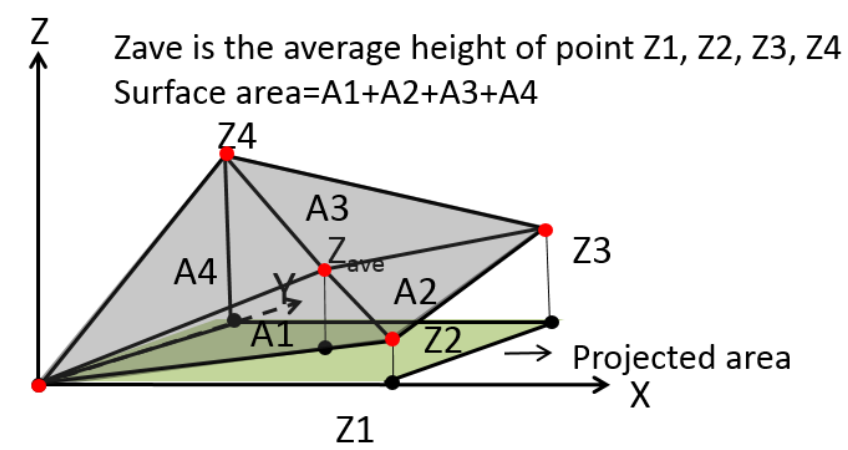

Figure 2.5. The schematic of 1 by 1 pixel surface area calculation, the red spot indicates point's height, a gray area indicates the surface area, and green area indicates projected area.

\section{3: Multifunctional Scanning Ion Conductance Microscopy (SICM)}

The SICM was integrated as a multifunctional Scanning Ion Conduction which can map topography and potential distribution of the sample at the same time. An illustration 
of the multifunctional SICM experimental setup has shown in chapter 6 in Figure 6.1a. Double barrel quartz theta nanopipette was used as a multifunctional probe. The Same electrolyte solution was filled into the both barrel of the theta pipette as well as outside bath solution. $\mathrm{Ag} / \mathrm{AgCl}$ electrodes were inserted into both electrolyte-filled barrels. One barrel was used for the position control of the nanopipette by sensing the ionic current. The other barrel was used for the potential measurement on the same reference electrode $(\mathrm{Ag} / \mathrm{AgCl})$ used for the ionic current measurement which was immersed in the outside bath electrolyte. A home-built (prepared by the electronic shop of FIU physics), battery powered high input impedance (with almost zero current flow through the circuit) differential amplifier was used to measure the potential difference.

The high-quality probe is always very important for all the SPM techniques. Multifunctional nanopipettes were fabricated and characterized to enhance the sensitivity of the potential measurement. The following subsections explain multifunctional probe fabrication and characterization for the multifunctional SICM. Multifunctional nanopipettes from quartz theta capillary tubes were fabricated as explained in section 2.1.2.

\subsection{1: Pyrolytic Carbon Nanoelectrode Fabrication.}

Figure 2.6 shows the schematic setup for the fabrication of pyrolytic carbon nanoelectrode $(\mathrm{CNE})$ from the theta nanopipette. Previously reported fabrication method of pyrolytic CNE was followed and modified slightly according to our intend. ${ }^{9}$ The back of one barrel of theta nanopipette was blocked with a removable plug (Blu-tack) to prevent carbon deposition, and butane gas flowed through the opened barrel of a dual-nanopore nanopipette. The taper of the theta nanopipette was inserted into another quartz pipette (O.D $1.0 \mathrm{~mm}$ and I.D. $0.7 \mathrm{~mm}$; Sutter Instrument). Argon flow was passed through the 
protection quartz pipette to prevent oxidation of the formed CNE tip and the bending of the nanopipette tip at high temperature. Home-built precision pressure meters were used to monitor the argon and butane gas flow. The pressures for argon and butane gas flow are $0.5 \mathrm{kPa}$ and $25 \mathrm{kPa}$ respectively. The nanopipette tip was heated with a butane torch (Blazer) for 30-40 seconds to form a solid pyrolytic carbon nanoelectrode. The prepared CNEs were investigated by an optical microscope, cyclic voltammetry (CV) and scanning electron microscope (SEM).

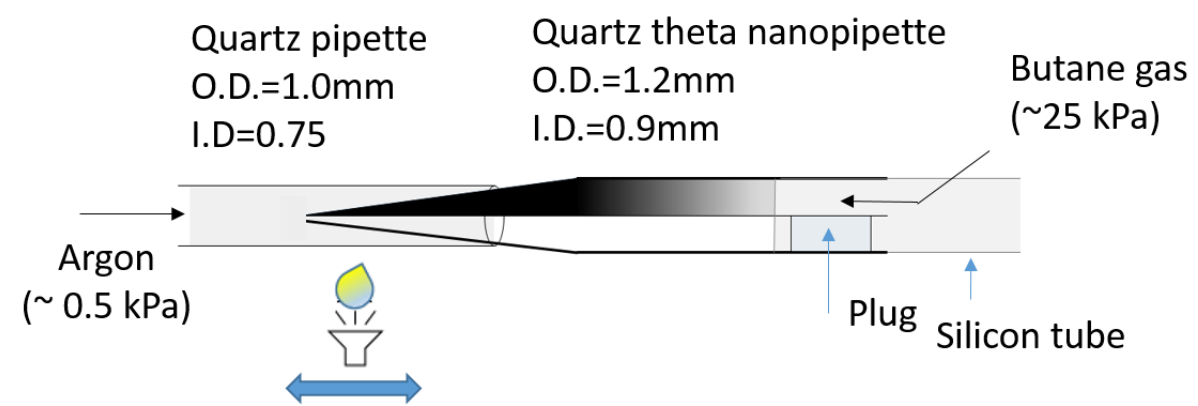

Figure 2.6. The Schematic setup for the fabrication of CNE from the theta nanopipette.

\subsection{2: Characterization of Carbon-Nanoelectrode with Cyclic Voltammetry (CV) Meathod} Cyclic voltammetry (CV) was performed to assess the size and quality of the carbon nanoelectrodes (CNEs). The CV measurements were made using a three-electrode cell using a potentiostat (CHI760D, CH Instruments, Inc., USA). A coper wire coated with silver paint was inserted into the $\mathrm{CNE}$ barrel to make secure contact with the CNE which was the working electrode. $\mathrm{Ag} / \mathrm{AgCl}$ wire electrode was used as a quasi-reference electrode, and spirally coiled Platinum wire $(0.25 \mathrm{~mm}$ in diameter) was used as the counter 
electrode. The counter electrode was cleaned by sonication in DI water and hydrogen flaming before its use. Typically sigmoidal shaped steady-state CVs was observed from the fabricated CNEs as shown in Figure 2.7. The diffusion limited current $i_{d}$ of the CV was used to evaluate the CNE size. The CV of the CNE was collected in 1x PBS solution containing $1 \mathrm{mM} \mathrm{Ru}\left(\mathrm{NH}_{3}\right)^{6+}$ ions by cycling the electrode potentials at $20 \mathrm{mV} / \mathrm{s}$.

The size of CNEs was calculated from the following formula.

$i d=\mathrm{mFDC} \sqrt{2 \pi A_{\text {eff }}}$,

where $\mathrm{m}$ is a geometry factor, $\mathrm{F}$ is the Faraday constant $(96485 \mathrm{C} / \mathrm{mol}), \mathrm{D}$ and $\mathrm{C}$ are the diffusion constant $\left(7.4 \times 10^{-6} \mathrm{~cm}^{2} / \mathrm{s}\right)$ and the bulk concentration of $\mathrm{Ru}\left(\mathrm{NH}_{3}\right)^{6+}$ ions. The geometry factor $m=1$ if the electrode is hemispherical and $m$ changes slightly (normally less than 10\%) for other geometries. For example, $m=1.1$ if the aspect ratio of an oblate hemispheroid is 6. The SEM images revealed the aspect ratios of CNEs were in the range of 2-4. Therefore, $m=1$ was used for the estimation of $A_{\text {eff. }}$.

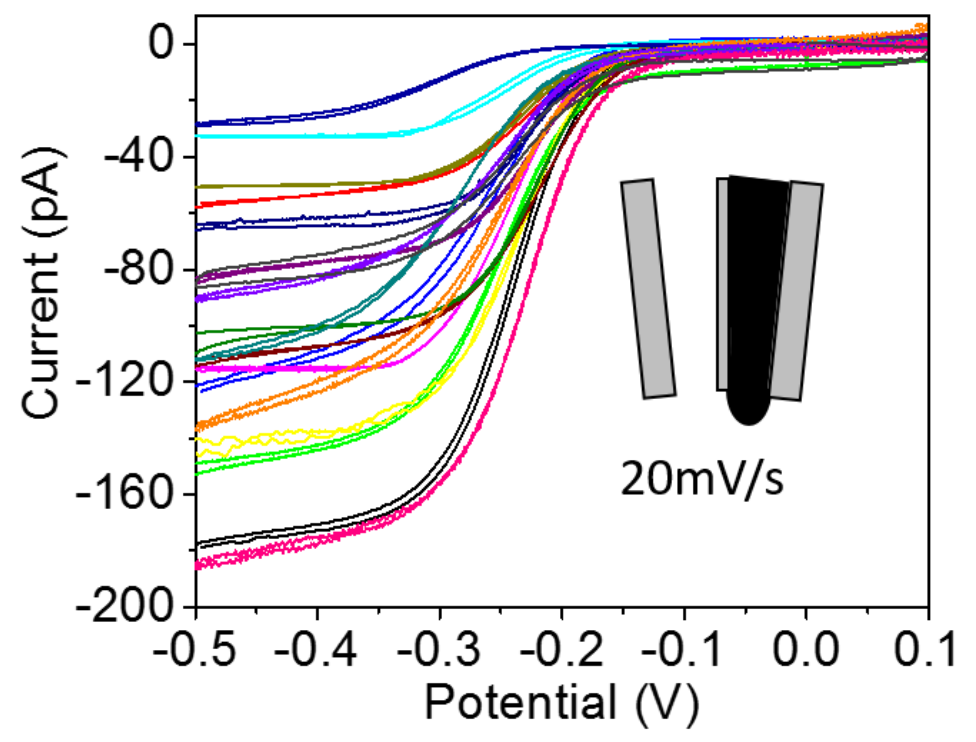

Figure 2.7 The steady-state CVs (at a sweep rate $20 \mathrm{mV} / \mathrm{s}$ ) for $18 \mathrm{CNEs}$ in $1 \mathrm{x}$ PBS solution containing $1 \mathrm{mM} \mathrm{Ru}\left(\mathrm{NH}_{3}\right)^{6+}$ ions. 


\subsection{3: Measurement of Nanopore Surface Charge}

As reported previously, ${ }^{4,5}$ current-voltage (I-V) curves were used to estimate the surface charge of inner nanopore surface. As a consequence of the conical shaped geometry and the surface charge of quartz material, asymmetry shaped IV curves were observed. Assuming a fixed half-cone angle of the nanopipette, those asymmetric IVs were used for the estimation of surface charge density of the nanopore, which was quantified by the ion current rectification ratio as $\mathrm{r}=\log \left|\frac{\mathrm{I}_{+}}{\mathrm{I}_{-}}\right| \cdot{ }^{10}$ After the fabrication of $\mathrm{CNE}$, the magnitude of the rectification ratio $r$ was reduced.

\subsection{4: Data Collection}

The ionic current-time (I-t) and potential-time (V-t) traces were recorded using the experiment setup shown in Figure 2.8. The setup was housed in a home-built Faraday cage on an air floating optical table to reduce electrical and mechanical noise. One $\mathrm{Ag} / \mathrm{AgCl}$ wire electrode was placed inside the nanopipette, and one was placed in the bath. The bath side was always grounded. The I-t traces were collected at various voltages by Axon 200B (Molecular Devices Inc., CA) in voltage clamp mode. The potential change at the CNE was measured by a home-built, battery powered high input impedance differential amplifier (based on an instrumentation amplifier) at a 10x gain. A digital oscilloscope (Yokogawa DL850 scopecorder) was used to record the current and potential traces with a sampling rate $50 \mathrm{kHz}$. The potential data noise at high frequency was much smaller than the current data. Thus, the low-pass filter bandwidth is $5 \mathrm{kHz}$ for current and $40 \mathrm{kHz}$ for potential. All the measurements were performed at room temperature. 


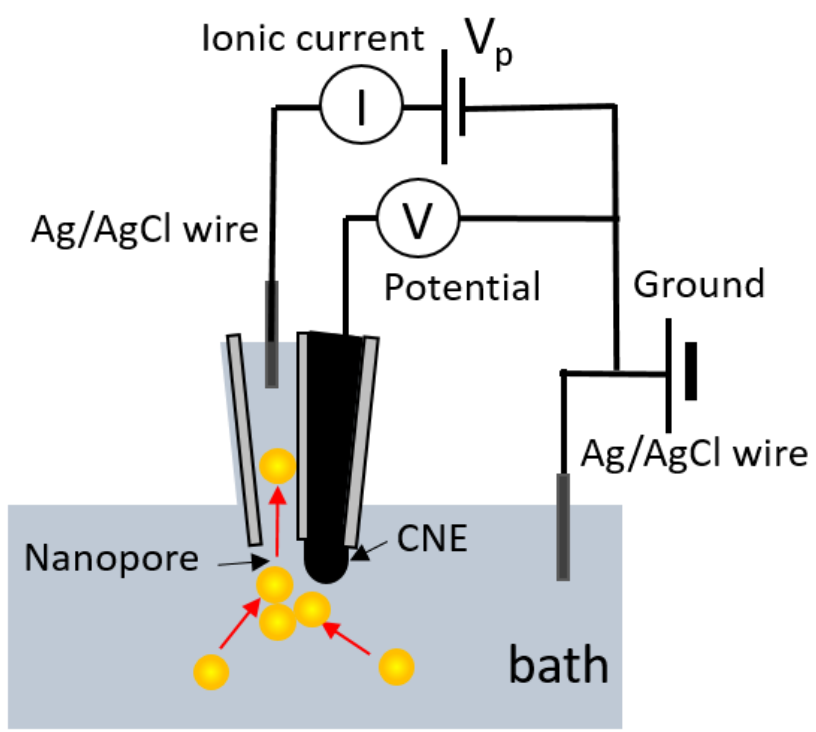

Figure 2.8. The schematic experimental setup of using nanopore/CNE nanopipette for simultaneous current and potential measurements of the GNP motion in the bath solution. $\mathrm{V}_{\mathrm{p}}$ is the applied bias. The potential is measured by a high impedance voltage meter.

2.3.5: Zeta Potential Measurement of $40 \mathrm{~nm}$ GNPs with Dynamic Light Scattering (DSL) Technique

The zeta potential measurement of $40 \mathrm{~nm}$ GNPs was performed by Zetasizer nanoZS (Zen 3600, Malvern Instruments Ltd.) using a folded capillary cell (Catalog \# DTS1070) at room temperature. Each sample was analyzed six times. The zeta potential of GNP depends on solution salt concentration. The zeta potential of $40 \mathrm{~nm}$ GNPs in10 mM PBS solution at $\mathrm{pH} 7.4$ was $-34.2 \mathrm{mV}$. The zeta potential was changed to $-41 \mathrm{mV}$ at $5 \mathrm{mM}$ PBS solution at pH 7.4. According to Ted Pella Inc. (the supplier of $40 \mathrm{~nm}$ GNP), the zeta potential was $-44 \mathrm{mV}$ in $\mathrm{DI}$ water.

The measured zeta potential is at the slipping plane of the GNP. At low salt concentration, we can ignore the stern layer. The slipping plane thickness X can be calculated with the following formula: ${ }^{11}$

$$
\chi_{\mathrm{sp}}=\frac{\ln \frac{\zeta_{1}}{\zeta_{2}}}{\left(\frac{1}{\delta_{2}}-\frac{1}{\delta_{1}}\right)}
$$


where $\zeta_{1}$ and $\zeta_{2}$ are the zeta potentials, and $\delta_{2}$ and $\delta_{1}$ are the Debye lengths of the $5 \mathrm{mM}$ and $10 \mathrm{mM}$ PBS solutions respectively. For the GNPs used in the present experiment, $\zeta_{1}=$ $-41 \mathrm{mV}, \zeta_{2}=-34 \mathrm{mV}, \delta_{1}=4.02 \mathrm{~nm}$ and $\delta_{1}=2.84 \mathrm{~nm}$ for $5 \mathrm{mM}$ and $10 \mathrm{mM}$ PBS solutions. The slipping plane thickness was estimated to be $\sim 1.9 \mathrm{~nm}$. Using the slipping plane thickness, the GNP surface potential $V_{0}$ was calculated with the following formula:

$$
\mathrm{V}_{0}=\mathrm{V}_{\mathrm{sp}} \mathrm{e}^{\chi_{\mathrm{sp}} / \delta},
$$

where $V_{\text {sp }}$ is the potential of GNP at the slipping plane or the measured zeta potential. We got $\mathrm{V}_{0}=-66 \mathrm{mV}$. Using the Grahame equation, the corresponding surface charge density $\sigma_{0}$ of GNP in $10 \mathrm{mM}$ PBS was calculated to be $-24 \mathrm{mC} / \mathrm{m}^{2}$.

\subsection{6: Data Analysis}

The data were analyzed by home-built Labview programs and Originpro 2015. Moving average smoothing method with a $0.2 \mathrm{~ms}$ time window is typically applied to the current and potential results before statistical analysis. As shown in the figure5.11 (f), the smoothing did not alter the duration and height of the fast current and potential changes. The $\mathrm{dV} / \mathrm{dt}$ curves were smoothed by the moving average method using a $2 \mathrm{~ms}$ time window.

\subsection{7: Noise Analysis of Ionic Current and Potential Measurements}

The noise in ionic current and potential measurement data were analyzed and compared. The noise power spectrum density (PSD) S(f) was obtained by performing Fast Fourier Transformations (FFT) on a current and potential time trace of one second time duration.). The noise of potential data was much smaller than that of ionic current data, especially at high frequency $(>100 \mathrm{~Hz})$. 


\subsection{8: Finite Element Based Numerical Simulations}

Finite element (FEM) based numerical simulations were carried out to solve coupled Poisson-Nernst-Planck (PNP) partial differential equations. The fluidic flow term was not included, and the system was assumed at a steady state to simplify the simulation. COMSOL Multiphysics 5.2 with AC/DC and Chemical Reaction Engineering modules were used for the FEM simulation. The whole computation domain was discretized into free triangular elements, and rigorous mesh refinements were adopted during simulations.

2.4: Measuring Extracellular Potential Distributions of a Single Living Cell Membranes by Multifunctional SICM

The multifunctional SICM was developed as explained in section 2.3. The double barrel nanopipette was used as the probe. The sample stage was designed, and the favorable environment was maintained during the living cell experiments.

\subsection{1: New Sample Stage for Living Cell Experiment}

Commercially available sample stage (from park system) was replaced by a home build metal sample stage along with new sample holder as shown in Figure 2.9. Two heating element was inserted on both sides of the sample stage to apply temperature on it during living cell experiment. It should be noted that most of the mammalian cells grow at $35-37{ }^{\circ} \mathrm{C}$. Therefore maintaining the temperature within that range is very important for living cell experiment. The temperature was applied and controlled by the TC-1-100s temperature controller during the living cell experiment. 


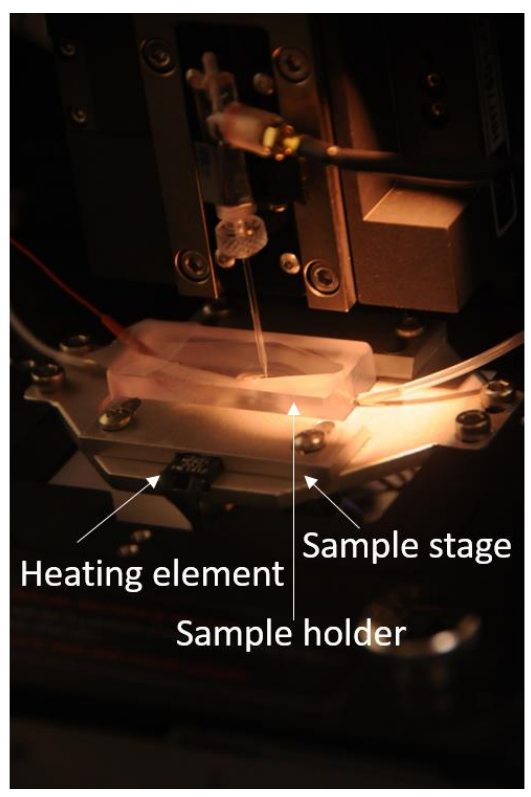

Figure 2.9 Living cell imaging sample stage

\subsection{2: Data Collection}

Extracellular membrane potential distribution of the living cell was recorded with single point measurement as well as by simultaneous mapping of topography and potential images of the certain area from a single cell. The whole SICM set up was housed on an inverted optical microscope (Nikon Eclipse Ti-U) to locate the theta nanopipette tip above the desired region of the cell of interest, and the optical images were captured by a CCD camera (UEye). The whole system was placed on air floated optical table to reduce mechanical noise.

\subsection{3: Data Analysis and Image Processing}

The data were analyzed by home-built Labview programs and Originpro 2015. Moving average smoothing method with a $20 \mathrm{~ms}$ time window is typically applied to the potential curves. The difference between the two potential curves recorded at two different height above the same location of the cell was used to calculate the real potential 
distribution of the cell membrane during fixed point measurement. Matlab will be used for the image processing.

2.4.4: Finite Element Analysis of Potential Measurement.

The potential distribution pattern was investigated using a FEM for solving Poisson and Nernst-Planck equations. A 2D axis symmetry geometry model was created to mimic the potentiometric SICM setup with theta pipette. The PNP equations were fully coupled and solved using boundary conditions matching the expected experimental conditions. The

fluidic flow term was not included, and the system was assumed at a steady state to simplify the simulation, COMSOL Multiphysics 5.2 with AC/DC and Chemical Reaction Engineering modules were used for the FEM simulation. The whole computation domain was discretized into free triangular elements, and rigorous mesh refinements were adopted during simulations.

\section{5: References}

1. Novak, P., Li, C., Shevchuk, A., Stepanyan, R., Caldwell, M., Hughes, S., Smart, T., Gorelik, J., Ostanin, V., Lab, M., Moss, G., Frolenkov, G., Klenerman, D. \& Korchev, Y. Nat Methods 2009, 6, 279

2. Shevchuk, A.I., Frolenkov, G.I., Sánchez, D., James, P.S., Freedman, N., Lab, M.J., Jones, R., Klenerman, D. \& Korchev, Y.E. Angewandte Chemie International Edition 2006, 45, 2212.

3. Wang, H., Hao, X., Shan, Y., Jiang, J., Cai, M. \& Shang, X. Ultramicroscopy 2010, 110,305 .

4. Wang, Y., Kececi, K., Mirkin, M.V., Mani, V., Sardesai, N. \& Rusling, J.F. Chemical Science 2013, 4, 655.

5. Tiwari, P.B., Astudillo, L., Miksovska, J., Wang, X., Li, W., Darici, Y. \& He, J. Nanoscale 2014, 6, 10255. 
6. Vlassiouk, I., Smirnov, S. \& Siwy, Z. Nano Letters 2008, 8, 1978.

7. Semwogerere, D. \& Weeks, E.R. 2005.

8. Nečas, D. \& Klapetek, P. Central European Journal of Physics 2012, 10, 181.

9. Takahashi, Y., Shevchuk, A.I., Novak, P., Zhang, Y., Ebejer, N., Macpherson, J.V., Unwin, P.R., Pollard, A.J., Roy, D., Clifford, C.A., Shiku, H., Matsue, T., Klenerman, D. \& Korchev, Y.E. Angewandte Chemie International Edition 2011, 50,9638 .

10. Vilozny, B., Actis, P., Seger, R.A., Vallmajo-Martin, Q. \& Pourmand, N. Anal. Chem. 2011, 83, 6121.

11. Shan, X., Fang, Y., Wang, S., Guan, Y., Chen, H.-Y. \& Tao, N. Nano Letters 2014, 14,4151 . 


\section{CHAPTER 3: CHARACTERIZATION OF THE POLYMER NANOPARTICLES WITH ATOMIC FORCE MICROSCOPY}

One of the major goals of my dissertation was to study the morphological changes on the cell membrane during the internalization of the nanoparticles. However, before treating the CPNs with the cell membrane, it is very important to characterize the size and shape of these synthesized polymer nanoparticles. Although we have used SICM for the study of the polymer nanoparticles interactions with the cell membrane, we choose AFM to characterize the polymer nanoparticles itself because it has higher resolution than the SICM. This chapter presents a characterization of the polymer nanoparticles with Atomic Force Microscopy. Some of the contents of this chapter have been adapted from my research work published in a peer-reviewed paper. ${ }^{1}$

\section{1: Introduction}

Conjugated polymers (CPs) are organic macromolecules which are characterized by an alternating single and double (or triple) bonds along a chain of carbon atoms on its backbone. There exist fully conjugated $\pi$-electrons because of the result of overlapping porbitals which can produce very interesting and useful optical and electronic properties. ${ }^{2,3}$ Conjugated polymers are naturally fluorescent materials that have very wide applications in various fields such as optoelectronics, photonics, bio-imaging, ${ }^{4-8}$ bio-sensing ${ }^{9-12}$, and nanomedicine ${ }^{13-15}$. Recently, conjugated polymers (CPs) have attracted much attention for various biological applications including imaging, sensing, and delivery of biologically active substances because of their excellent photo-physical and biophysical properties. They not only can be synthesized in desired sizes, shapes and properties but also are 
biocompatible and non-toxic which make these materials highly attractive for biological applications.

For biological applications, conjugated polymer nanoparticles have been synthesized by including hydrophilic polar side chains and functional entities such as sensing units or targeting ligands. ${ }^{16-18}$ Functional modifications and structural modulations of CP-based nanomaterials expected to obtain desired biophysical properties for better applications in cell biology such as drug delivery. But, particle size, morphology, composition, and surface area are important factors, which need to be addressed accurately to evaluate nanoparticle toxicity. ${ }^{19}$ It is very important and the first step to characterize size, shape and biophysical properties of CPNs before apply for its targeted applications

This chapter mainly focuses on the characterization of the CPNs's size shape and the morphology with Atomic Force Microscopy (AFM). Several techniques such as dynamic light scattering, laser diffraction, and X-ray diffraction have been used to measure the nanoparticle size distribution. ${ }^{19,20}$ 21-23 These techniques can provide the information of the average size distribution of a large number of particles but cannot provide the size, shape, and morphology of individual nanoparticles. Scanning electron microscopy $(\mathrm{SEM})^{24}$ and transmission electron microscopy (TEM) ${ }^{25,26}$ are widely used techniques to characterize individual nanoparticles, but they also cannot give the height information. ${ }^{27}$ Atomic force microscopy (AFM) is very versatile and powerful scanning probe microscopy technique for studying varieties of samples such as synthetic and biological membranes, metals, polymers, and semiconductors at the nanoscale. ${ }^{28-30}$ The AFM is versatile because it generates not only three-dimensional topography images with angstrom scale resolution, but also provides various types of surface properties. The AFM uses a cantilever with a 
very sharp tip at its end which is used to scan over a sample surface. As the tip approaches the sample surface, the force between the tip and the sample results into a deflection of the cantilever. The deflection of the cantilever reveals the surface topology of the sample with nanometer precision. The AFM can be operated in both liquid and air imaging. For soft biological samples, tapping mode imaging is preferred to contact and non-contact imaging mode. ${ }^{31-33}$ Hence, tapping mode imaging was used to characterize soft CPNs. Tapping mode not only acquires high-resolution topography images of the soft samples but also records the phase images by mapping the phase shift of the cantilever oscillation during tapping mode scanning. Phase images were used to extract extra information about the CPNs structure such as hard and soft region.

There is a hypothesis that if CPs containing flexible units along the backbones are treated with polymeric acids, $\pi-\pi$ interaction among the backbones will be dramatically increased because the semiflexibility of the nonaqueous soluble backbones will help backbone restructuring to maximize hydrophobic interactions. If complexation between the nonaqueous soluble $\mathrm{CP}$ and polyanion contributes to increase aqueous solubility of $\mathrm{CPs}$, random complex formation is expected. To test the hypothesis and functionalize the CP with cancer cell-specific ligands, a linear polysaccharide, hyaluronic acid (HA) containing N-acetyl-D-glucosamine and D-glucuronic acid units were used. HA has specific binding with cell surface receptors such as CD44 and RHAMN, which are overexpressed in many cancer cells. ${ }^{34}$ Many cancer drugs and polymers have been modified with HA for targeted drug and gene delivery. ${ }^{35-39}$ A semiflexible poly (phenylenebutadiynylene) (PPB), which contains a small fraction of flexible nonconjugated units called linker along with the rigid conjugated backbones, was 
complexed with hyaluronic acid (HA. Positively charged CPNs containing flexible units along the backbones when treated with hyaluronic acid (HA) will reorganize and form a core-shell structure nanoparticles wrapped by HA from outside as shown in schematic representation in Figure 3.1, while nonflexible CPs produce random complexes upon HA treatment. The core--shell nanoparticles are nontoxic to cells and exhibit high cancer cell specificity through the specific binding of HA to cancer cell surface receptors. The PPB/HA complex was formed by mixing PPB $(10 \mu \mathrm{M})$ with various molar equivalents of HA (i.e., 1:1, 1:3, and 1:9) for $1 \mathrm{~h}$. How the variations of the HA proportion play a role in the formation of the core-shell nanoparticles was studied with AFM. As a control, CP (PPE) without flexible units along its rigid conjugated backbone were treated with different proportions of HA in a similar fashion like with PPB and imaged. The structure of the PPE and HA is shown in Figure 3.2

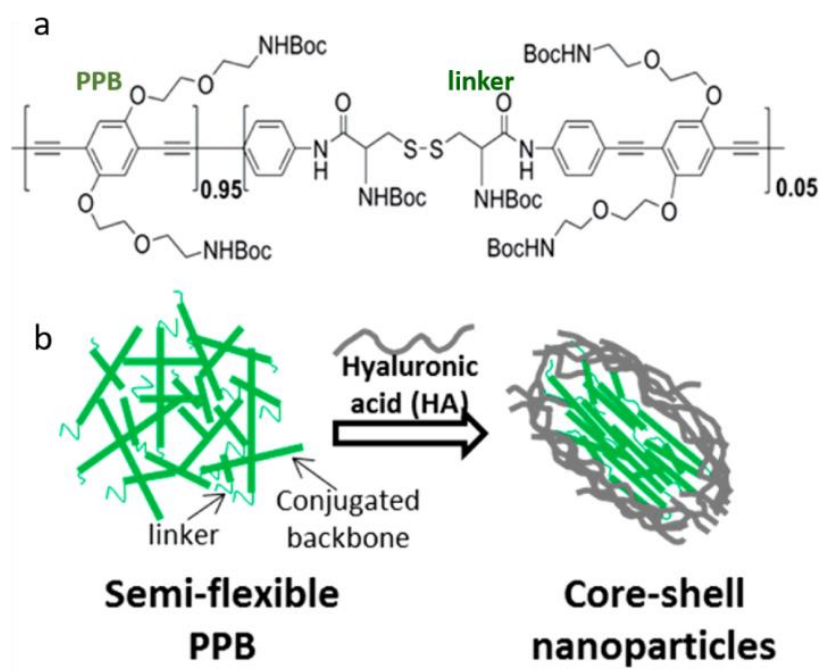

Figure 3.1. (a) Chemical structure of the semiflexible PPB. (b) A schematic presentation of structural reorganization of the semiflexible PPB upon HA complexation. 

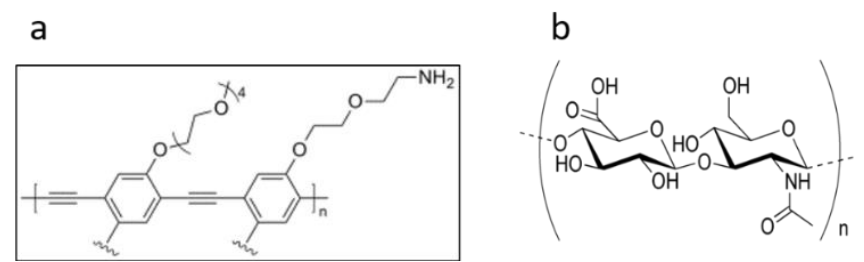

Figure 3.2. (a) Chemical structure of PPE without flexible units along its rigid conjugated backbone. (b) Chemical structure of hyaluronic acid.

\section{2: Methods}

Detailed about the sample preparation method and imaging condition was described in chapter 2 under section (2.1)

\section{3: Results and Discussion}

Nanoparticle supported the formation of elongated core-shell nanoparticles, as shown in Figure 3.3c, while the semiflexible PPB without HA complexation exhibited mixed particles with no specific shapes (Figure 3.3 a). The AFM phase image also showed that core-shell particles were favorably formed at a molar ratio of 1:3 of the semiflexible PPB to HA (Figure 3.3 f). Both at the lower and higher ratios, no defined core-shell nanoparticles were observed Figure ( $3.3 \mathrm{~b}$ and e). Higher density (represented as dark color) was observed in the center of the nanoparticles, while lower density (bright color) was observed in the shell. Since the particles were prepared on an aminosilanized mica surface, it is difficult to determine the shape and size of the intact nanoparticles in water by the AFM imaging. The lateral size and height of individual nanoparticles formed with the complexation of different proportion of HA were measured and plotted the histogram (Figure 3.4). The AFM images were flattened with first order polynomial during the height analysis. The lateral size and height of core -shell nanoparticle were $58 \pm 13 \mathrm{~nm}$ and $7.1 \pm 0.9$ respectively. 

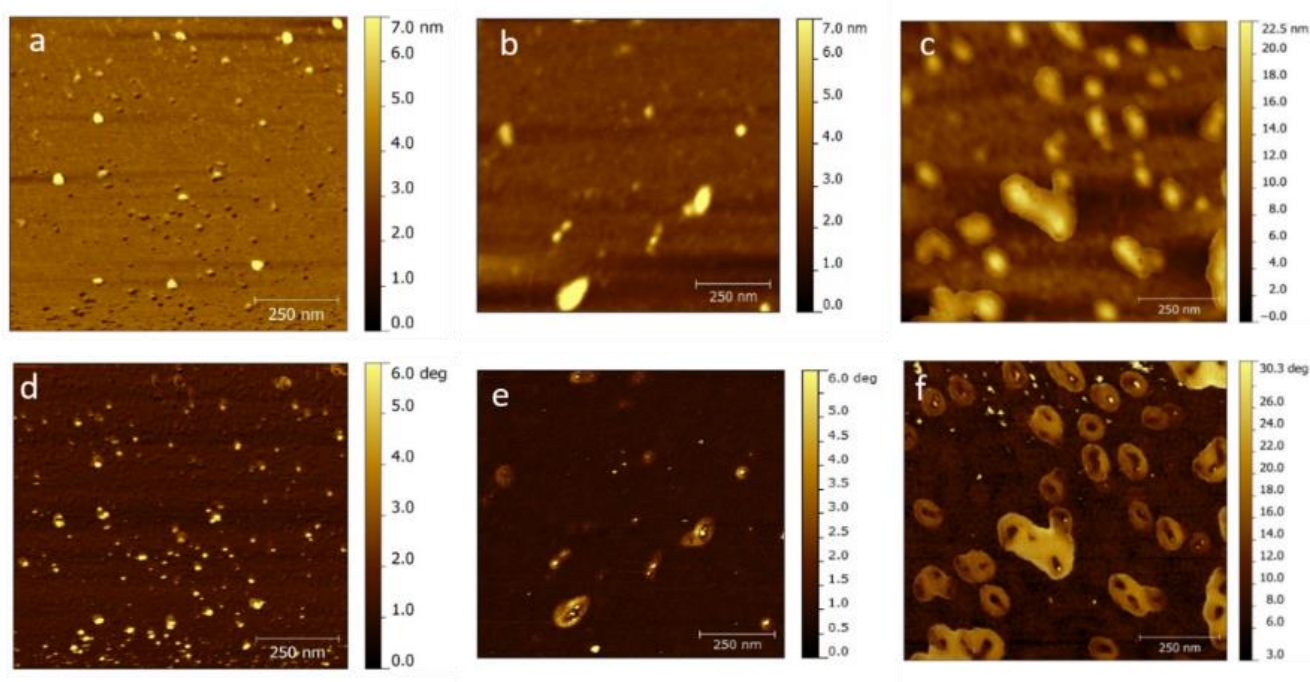

Figure 3.3. AFM topographic (a, b, c) and phase (d, e, f) images of the semiflexible PPB $(\mathrm{a}, \mathrm{d})$, semiflexible $\mathrm{PPB} / \mathrm{HA}$ nanoparticles formed at 1:1 molar ratio $(\mathrm{b}, \mathrm{e})$ and semiflexible PPB/HA nanoparticles formed at 1:3 molar ratio (c, f). The semiflexible PPB/HA with 1:3 molar ratio exhibits elongated particles on a mica surface (c), and the phase image (f) reveals that the complexes are core-shell nanoparticles.
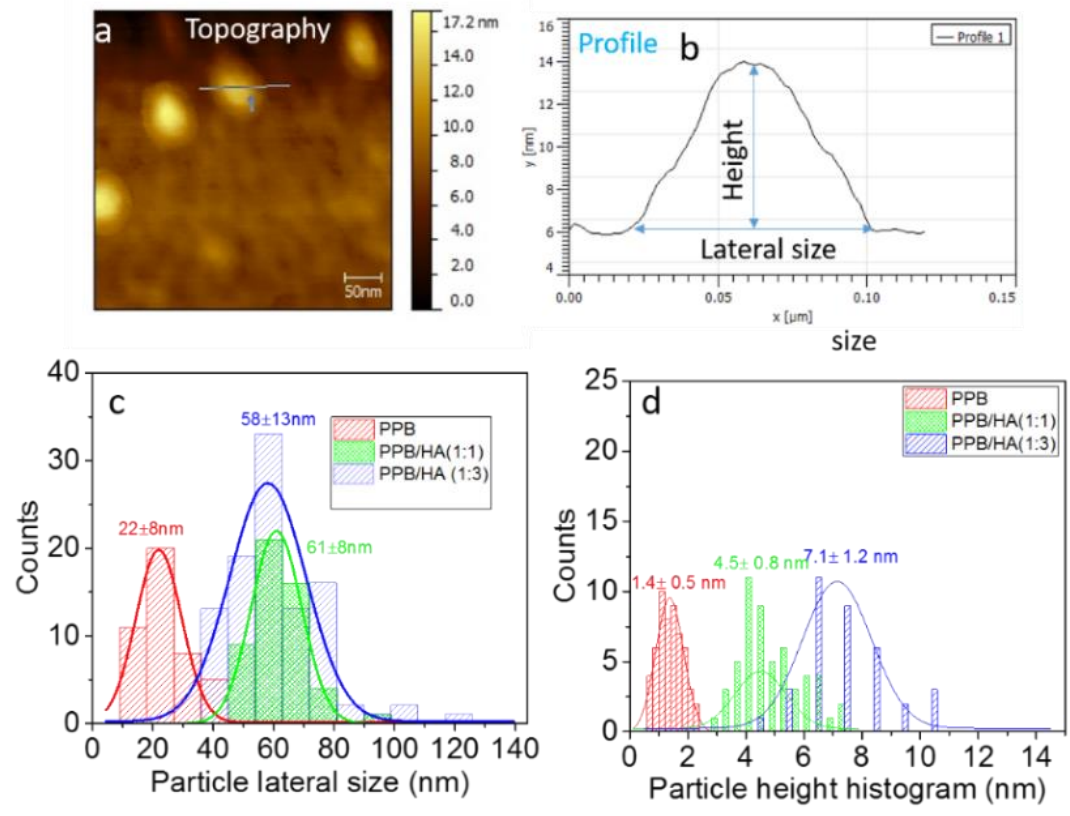

Figure 3.4 (a) Zoom in topography image of PPB/HA (1:3). (b) Profile of line 1 drawn above the nanoparticle on topography image (c) lateral size (d) height analysis histograms of semi-flexible PPB (red), semi-flexible PPB/HA (1:1) (green), and semi-flexible PPB/HA (1:3) (blue) respectively. 
The topographic and phase images of the control PPE/HA (1:3) show circular particles with no core-shell shape, with an average size and height of $81 \pm 9$ and $4 \pm 0.5$ nm, respectively (Figure 3.5). The AFM imaging of the control PPE/HA further supports that HA does not cause structural reorganization of rigid rod CPs.
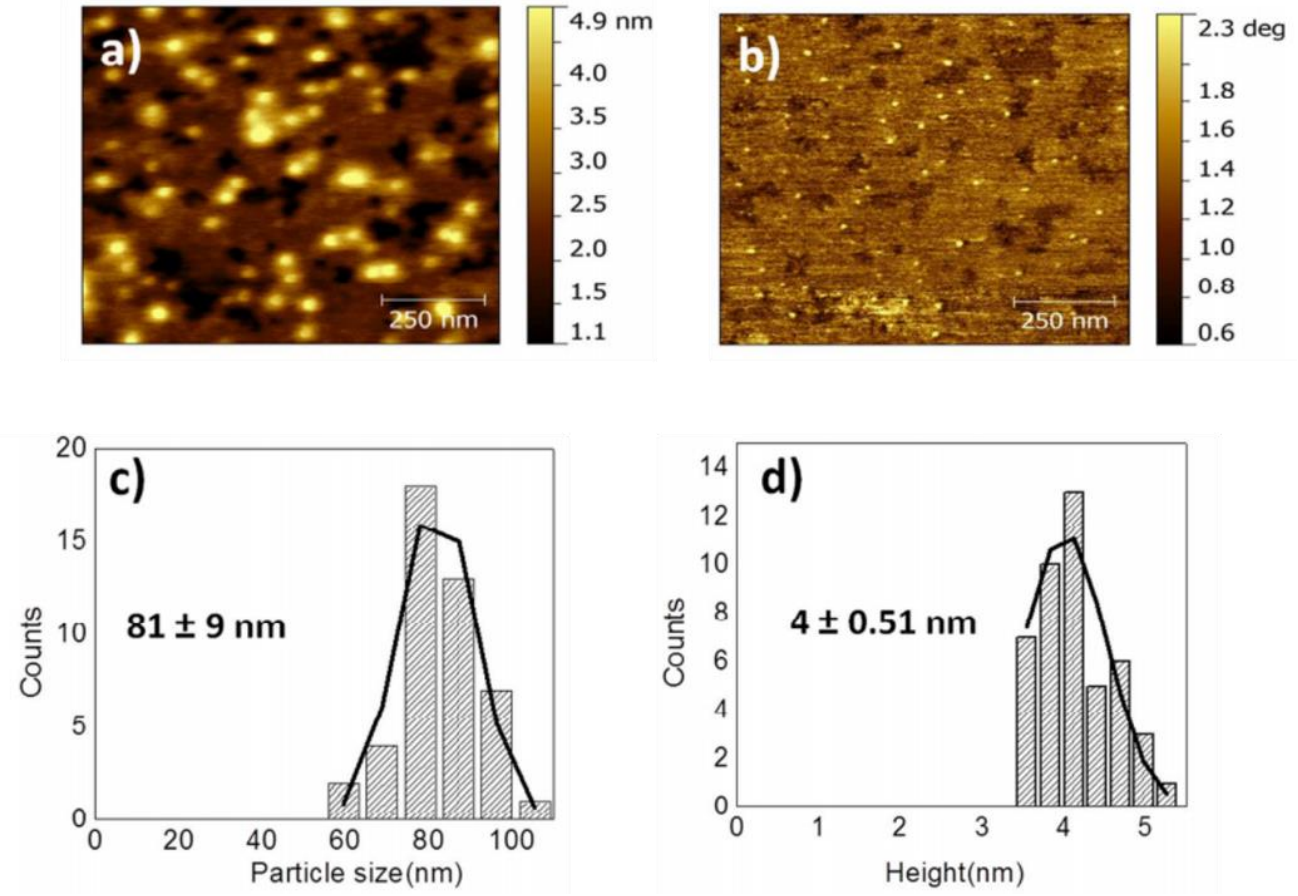

Figure 3.5. (a) Topography and (b) phase images, (c), size distribution and (d) height histograms for control PPE/HA.

Fabricated core-shell nanoparticles were very soft which can be declared from the Figure 3.6. During the same samples, slightly increasing imaging force can change the shape of these nanoparticles. As shown in figure 3.6 nanoparticles imaging with higher force changes its shape slightly and looks like donut shape but when decreasing the imaging force donut-like structure disappears. Simultaneously recorded phase image further confirms the soft nature of CPNs. 

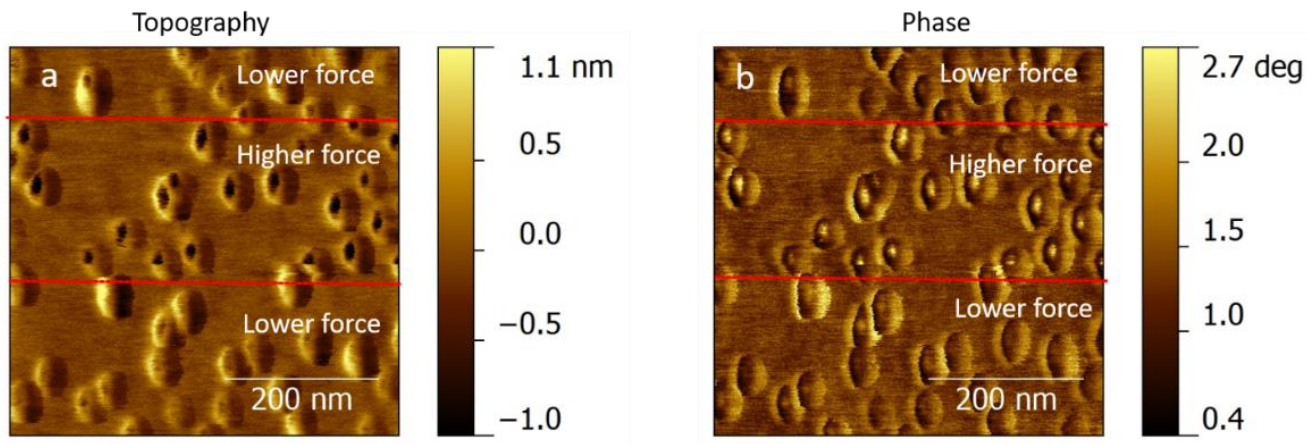

Figure 3.6. (a) Topography image and (b) Phase image of CPN/HA (1:1).

\section{4: Conclusion}

In summary, the formation of core-shell nanoparticles by complexing a semiflexible PPB with a linear polysaccharide, HA was clearly observed by AFM imaging. Its size and shape were also characterized. Since the size and shape of nanomaterials significantly influence labeling and delivery efficiency of biological substances, the synthesis of core-shell nanoparticle will contribute to novel biomaterials especially for drug delivery.

\section{5: References}

1. Twomey, M., Na, Y., Roche, Z., Mendez, E., Panday, N., He, J. \& Moon, J.H. Macromolecules 2013, 46, 6374.

2. Bunz, U.H. Accounts of chemical research 2001, 34, 998.

3. Roncali, J. Chemical Reviews 1997, 97, 173.

4. Bajaj, A., Miranda, O.R., Kim, I.B., Phillips, R.L., Jerry, D.J., Bunz, U.H. \& Rotello, V.M. Proc Natl Acad Sci U S A 2009, 106, 10912.

5. Kim, I.B., Shin, H., Garcia, A.J. \& Bunz, U.H. Bioconjugate chemistry 2007, 18, 815. 
6. Moon, J.H., McDaniel, W., Maclean, P. \& Hancock, L.F. Angewandte Chemie (International ed. in English) 2007, 46, 8223.

7. Yu, J., Wu, C., Zhang, X., Ye, F., Gallina, M.E., Rong, Y., Wu, I.C., Sun, W., Chan, Y.H. \& Chiu, D.T. Advanced materials (Deerfield Beach, Fla.) 2012, 24, 3498.

8. Lee, K., Lee, J., Jeong, E.J., Kronk, A., Elenitoba-Johnson, K.S., Lim, M.S. \& Kim, J. Advanced materials (Deerfield Beach, Fla.) 2012, 24, 2479.

9. Pinto, M.R. \& Schanze, K.S. Proceedings of the National Academy of Sciences of the United States of America 2004, 101, 7505.

10. Gaylord, B.S., Heeger, A.J. \& Bazan, G.C. Proceedings of the National Academy of Sciences 2002, 99, 10954.

11. You, C.C., Miranda, O.R., Gider, B., Ghosh, P.S., Kim, I.B., Erdogan, B., Krovi, S.A., Bunz, U.H. \& Rotello, V.M. Nat Nanotechnol 2007, 2, 318.

12. Wang, L., Pu, K.Y., Li, J., Qi, X., Li, H., Zhang, H., Fan, C. \& Liu, B. Advanced materials (Deerfield Beach, Fla.) 2011, 23, 4386.

13. Moon, J.H., Mendez, E., Kim, Y. \& Kaur, A. Chemical communications (Cambridge, England) 2011, 47, 8370.

14. Silva, A.T., Nguyen, A., Ye, C., Verchot, J. \& Moon, J.H. BMC plant biology 2010, 10, 291.

15. Feng, X., Lv, F., Liu, L., Yang, Q., Wang, S. \& Bazan, G.C. Advanced materials (Deerfield Beach, Fla.) 2012, 24, 5428.

16. Thomas, S.W., 3rd, Joly, G.D. \& Swager, T.M. Chem Rev 2007, 107, 1339.

17. Zhu, C., Liu, L., Yang, Q., Lv, F. \& Wang, S. Chem Rev 2012, 112, 4687.

18. Pecher, J. \& Mecking, S. Chem Rev 2010, 110, 6260.

19. Murdock, R.C., Braydich-Stolle, L., Schrand, A.M., Schlager, J.J. \& Hussain, S.M. Toxicological sciences 2008, 101, 239.

20. Fisker, R., Carstensen, J.M., Hansen, M.F., Bødker, F. \& Mørup, S. Journal of Nanoparticle Research 2000, 2, 267.

21. Powers, K.W., Palazuelos, M., Moudgil, B.M. \& Roberts, S.M. Nanotoxicology 2007, 1, 42 . 
22. Bootz, A., Vogel, V., Schubert, D. \& Kreuter, J. European Journal of Pharmaceutics and Biopharmaceutics 2004, 57, 369.

23. Hoo, C.M., Starostin, N., West, P. \& Mecartney, M.L. Journal of Nanoparticle Research 2008, 10, 89.

24. Perevyazko, I., Vollrath, A., Hornig, S., Pavlov, G.M. \& Schubert, U.S. Journal of Polymer Science Part A: Polymer Chemistry 2010, 48, 3924.

25. Borchert, H., Shevchenko, E.V., Robert, A., Mekis, I., Kornowski, A., Grübel, G. \& Weller, H. Langmuir 2005, 21, 1931.

26. Srnová-Šloufová, I., Lednický, F., Gemperle, A. \& Gemperlová, J. Langmuir 2000, 16, 9928.

27. Hanauer, M., Pierrat, S., Zins, I., Lotz, A. \& Sönnichsen, C. Nano Letters 2007, 7, 2881.

28. Mühlen, A.z., Mühlen, E.z., Niehus, H. \& Mehnert, W. Pharmaceutical Research 1996, 13, 1411.

29. Rao, A., Schoenenberger, M., Gnecco, E., Glatzel, T., Meyer, E., Brändlin, D. \& Scandella, L. in Journal of Physics: Conference Series, Vol. 61971 (IOP Publishing, 2007).

30. Baalousha, M. \& Lead, J.R. Colloids and Surfaces A: Physicochemical and Engineering Aspects 2013, 419, 238.

31. Putman, C.A., Van der Werf, K.O., De Grooth, B.G., Van Hulst, N.F. \& Greve, J. Applied physics letters 1994, 64, 2454

32. Ebenstein, Y., Nahum, E. \& Banin, U. Nano Letters 2002, 2, 945.

33. Magonov, S.N. \& Reneker, D.H. Annual Review of Materials Science 1997, 27, 175.

34. Toole, B.P. Nature reviews. Cancer 2004, 4, 528.

35. Needham, C.J., Williams, A.K., Chew, S.A., Kasper, F.K. \& Mikos, A.G. Biomacromolecules 2012, 13, 1429.

36. Goh, E.J., Kim, K.S., Kim, Y.R., Jung, H.S., Beack, S., Kong, W.H., Scarcelli, G., Yun, S.H. \& Hahn, S.K. Biomacromolecules 2012, 13, 2554. 
37. Choi, K.Y., Yoon, H.Y., Kim, J.H., Bae, S.M., Park, R.W., Kang, Y.M., Kim, I.S., Kwon, I.C., Choi, K., Jeong, S.Y., Kim, K. \& Park, J.H. ACS Nano 2011, 5, 8591.

38. Li, F., Bae, B.C. \& Na, K. Bioconjugate chemistry 2010, 21, 1312.

39. Choi, K.Y., Chung, H., Min, K.H., Yoon, H.Y., Kim, K., Park, J.H., Kwon, I.C. \& Jeong, S.Y. Biomaterials 2010, 31, 106. 


\section{CHAPTER 4: SCANNING ION CONDUCTANCE MICROSCOPIC STUDY FOR CELLULAR UPTAKE OF CATIONIC CONJUGATED POLYMER NANOPARTICLES}

In Chapter 3, I discussed the characterization of the conjugated polymer nanoparticles with the AFM. Although AFM has high resolution than the SICM, it was difficult to figure out the real morphological changes on the cell membrane during the internalization of CPNs through the cell membranes. There were also some changes in the cell membranes due to the interaction between the soft cell membranes and sharp AFM tip. In this chapter we report, how scanning ion conductance microscopy (SICM) can help to reveal the changes in cell membrane morphologies during endocytosis of polymer nanoparticles. For the first time, the cell surface morphological changes of human cervical cancer cells (HeLa) treated with CPNs was imaged and quantified by using SICM technique. Our results clearly demonstrated that SICM is a suitable imaging technique to disclose the dynamic alternations on the cell surface morphology during the early stage of nanoparticles endocytosis with high resolution. The entire content in this chapter has been adapted from my research results published in a peer-reviewed paper ${ }^{1}$.

\section{1: Introduction}

Understanding the details of how viruses, bacteria and naturally occurring and synthetic nanoparticles (NPs) interact and penetrate cell membrane is essential in developing drug or gene delivery systems..$^{2-4}$ Several imaging methods have frequently been used to study cellular uptake of NPs. Optical microscopy and fluorescence optical microscopy remain the most widely used imaging methods. However, the optical imaging methods are still limited in resolving the features with dimensions of tens of nanometers, especially on the cell membrane. Electron microscopes (EMs) have been used to reveal the 
NPs at the cell surface and inside the cell. The spatial resolution of EM is very high, but the cells need to be fixed and dehydrated. Atomic force microscopy (AFM) has also been used to image the distribution of NPs at cell surfaces. However, it is only limited to cells with the rigid surface because of strong interactions between AFM probe and the sample., ${ }^{6}$ Therefore, we used SICM technique to image and study the dynamical process of NPs internalization with high spatial resolution.

Scanning ion conductance microscopy (SICM), a unique combination of patchclamp and scanning probe microscopic (SPM) techniques, has existed for more than 20 years. ${ }^{7}$ Due to the continuous improvements in feedback control system, SICM has emerged as a powerful tool for the imaging and analysis of fragile, adhesive or responsive surfaces, such as live cell membrane. ${ }^{7-9}$ The SICM can reveal tens of nanometer scale resolution topography imaging of living cell membranes. The sample preparation is also much simpler than EMs and living cell imaging is possible for a long time. For example, non-specific adsorbed virus-like particles were visualized at COS7 cell membranes by SICM. ${ }^{10}$ The plasma membrane morphology change associated with exocytosis were observed at the membranes of bovine chromaffin cells. ${ }^{11}$ The dynamics of microvilli (membrane projections) assembly in various epithelial and nonepithelial living cells have been revealed. ${ }^{12,13}$ SICM has also resolved the location, structure, and dynamics of single protein and protein complex in the cell membrane. ${ }^{14,15}$

Primary amine-containing conjugated polymer nanoparticles (CPNs) are intrinsic fluorescent materials fabricated by self-assembly of non-aqueous soluble $\pi$-electron conjugated polymers (CPs) in an aqueous solution. ${ }^{16,17}$ Owing to excellent photophysical and biophysical properties, CPNs have attracted growing interest in live cell imaging, drug 
delivery, and biosensing. ${ }^{18-20}$ Primary amine-containing positively charged CPNs enter human cervical cancer cells (HeLa) via various endocytosis pathways. The pathways are highly dependent on the chemical functionalities of both side chains and backbones, which influence the surface properties of CPNs, resulting in different cellular interaction and subsequent entry. ${ }^{21,22}$ It is believed that the hydrophobicity from the backbone and positive charge from the side chain allow efficient interaction with the cell membrane, which contains negatively charged proteoglycans and hydrophobic membrane lipids. It is very important to understand the details and fundamental mechanisms of endocytosis processes of CPNs. The study of how CPNs attach and interact with the cell membrane helps to achieve high cellular labeling, sensing, and delivery efficiency. Despite growing evidence of CPNs' endocytosis, there are few direct evidences how the cellular membrane initially responds to synthetic extracellular materials.

Herein, cell surface morphological changes in HeLa cells induced by CPNs were investigated using SICM technique. The synthesis and characterization of the specific type of CPN used here have been reported before. ${ }^{17}$ These CPNs are positively charged (from protonation of primary amines), and their hydrodynamic diameters in solution are $56 \mathrm{~nm}$, measured by dynamic light scattering. Cell viability assays indicate that CPNs are not toxic up to $40 \mu \mathrm{M}$. Fluorescence microscopy studies revealed that CPNs accumulated in the cytosol after overnight incubation. For short incubation time (i.e., 1h), CPNs appeared to attach to the cell membrane and form aggregates. Detailed and systematic studies of the morphological changes of HeLa cells surface at various concentrations and incubation times were carried out with SICM. When HeLa cells were incubated with CPNs for a short time (i.e., 1h), distinct cell surface morphology changes on the HeLa was discovered. The 
features with submicron to micron sizes of protrusions (similar to microvilli) and small pits were found on HeLa cell membrane. The morphological changes were dependent on the concentration of CPNs and the incubation time. These structures disappeared after further continuous incubation. Control experiments were also carried out with highly positively charged polyethyleneimine (PEI) with the same concentration and the incubation time. Little surface changes were induced with PEI. By comparing with a control experiment, the cell surface changes were attributed to possible adsorption of de-aggregated CPNs at the lipophilic cell membranes. Unlike PEI, CPNs distinctly exhibit high hydrophobic interaction with the hydrophobic membrane. This observation provides additional evidence that the hydrophobicity of polymeric materials is indeed an important contributor for efficient cellular interaction and following entry.

\section{2: Methods}

Some methods used to accomplish this project are described in chapter 2. Details about nanopipette fabrication and characterization are explained in the section 2.2.1. Similarly details about the confocal fluorescence imaging, data collection and analysis can also be found in chapter 2 under section 2.2.3 and 2.2.4 respectively. Rest of the methods and chemicals used in this project are explained in following subsections.

\subsection{1: Chemicals}

All chemicals and solvents were purchased and used without further purification.

CPNs were synthesized as previously reported. ${ }^{17}$ The one-micron size polystyrene microbeads (suspension, 5\% w/v in water) were purchased from Spherotech Inc. Branched polyethyleneimine (PEI Mw 25,000) was purchased from Aldrich. All other chemicals were purchased from Sigma-Aldrich unless mentioned otherwise. All solutions were 
prepared using deionized (DI) water $(\sim 18 \mathrm{M} \Omega)$ from water purification system (Ultra Purelab system, ELGA/Siemens). Phosphate buffered saline (PBS) at $\mathrm{pH} 7.2$ was prepared with the following composition in $\mathrm{mM}$ : $\mathrm{NaCl} 137, \mathrm{KCl} 2.7, \mathrm{KH}_{2} \mathrm{PO}_{4}$ 1.5, $\mathrm{Na}_{2} \mathrm{HPO}_{4} 4.3$.

\subsection{2: Cell Culture}

HeLa cells (human cervical carcinoma) purchased from the American Tissue Culture Center (ATCC) were cultured in a mixture of modified Eagle medium (MEM) and Earle's balanced salt solution (EBSS) containing 10\% fetal bovine serum and 1\% penicillin/streptomycin (Thermo Scientific) at $37{ }^{\circ} \mathrm{C}$ in a $5 \% \mathrm{CO}_{2}$ incubator. Cells were usually cultured for 1 day to achieve $50 \%$ coverage (ca. 125000 per well) on a cover glass slide.

\subsection{3: CPN and NP Incubation}

Solutions with various concentrations of $\mathrm{CPN}$ were prepared. For example, $10 \mu \mathrm{L}$ aqueous solution containing $1 \times 10-3 \mathrm{M} \mathrm{CPNs}$ was added into the cell culture wells to get the final concentration of $10 \times 10-6 \mathrm{M}$. The CPNs were incubated with cells for a different time at $37^{\circ} \mathrm{C}$, subsequently washed three times with PBS to remove the excess CPNs which did not interact with cells. Before SICM scanning, the cells were fixed with $4 \%(\mathrm{w} / \mathrm{v})$ paraformaldehyde for 10 min and washed three times with PBS. The samples were imaged on glass coverslips, supported by a Petri dish, in an appropriate medium. The bath solution used for fixed cell imaging was PBS. For actin inhibitor and metabolism inhibitor experiments, HeLa cells were first treated by $1 \times 10-6 \mathrm{M}$ cytochalasin D for $20 \mathrm{~min}$, and then were incubated in $10 \times 10-6 \mathrm{M} \mathrm{CPNs}$ for $1 \mathrm{~h}$. For metabolism inhibitor experiments, HeLa cells were first treated with $10 \times 10-3 \mathrm{M}$ sodium azide for $20 \mathrm{~min}$, and then were incubated in $10 \times 10-6 \mathrm{M} \mathrm{CPNs}$ for $1 \mathrm{~h}$. 


\section{3: Results and Discussion}

Because of the ability to internalize nanoparticles, ${ }^{23}$ HeLa cells were used to study the cell surface morphology change during endocytosis of CPNs. Although prolonged incubation (e.g., overnight incubation) of CPNs obviously provides evidence on endocytosed CPNs by HeLa cells, no knowledge on the initial cell response to short time CPN treatment (e.g., $1 \mathrm{~h}$ incubation) was available.Live cells treated with CPNs were fixed by $4 \%$ (w/v) paraformaldehyde just before SICM imaging to simplify the experiment. Live cells were also imaged (under no $\mathrm{CO}_{2}$ ), and the changes in surface morphology were found to be similar to those of fixed cells (see Figure 4.1). The cells imaged by SICM were preselected using the optical microscope. Only isolated cells adhered strongly to the glass substrate were selected, as shown in Figures 2.1. The selected cells exhibited similar volume, size, and shape. 

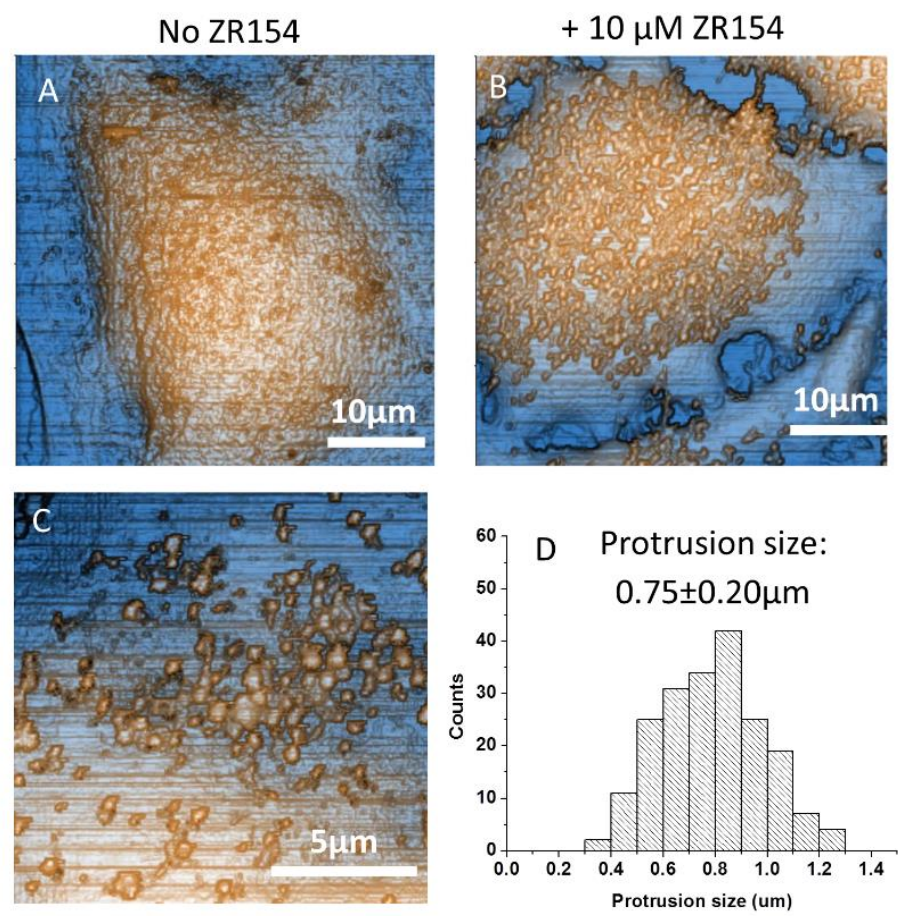

Figure 4.1. (a) The SICM image (enhanced color) of a live HeLa cells. (b-c) The SICM images (enhanced color) of live HeLa cells after treating with 10uM CPNs. (d) The histogram of the Protrusion height.

Similarly, a typical SICM topography image (with enhanced color) of fixed HeLa cells is shown in Figure 4.2. In both figure 4.1 and 4.2, the blue colored area is flat and smooth, and the orange colored area has bigger height variations. In this image, only a few protrusions and blebs were observed at the cell surfaces. A SICM topography images (without enhanced color) after flattering is also shown in Figure 4.2b. 

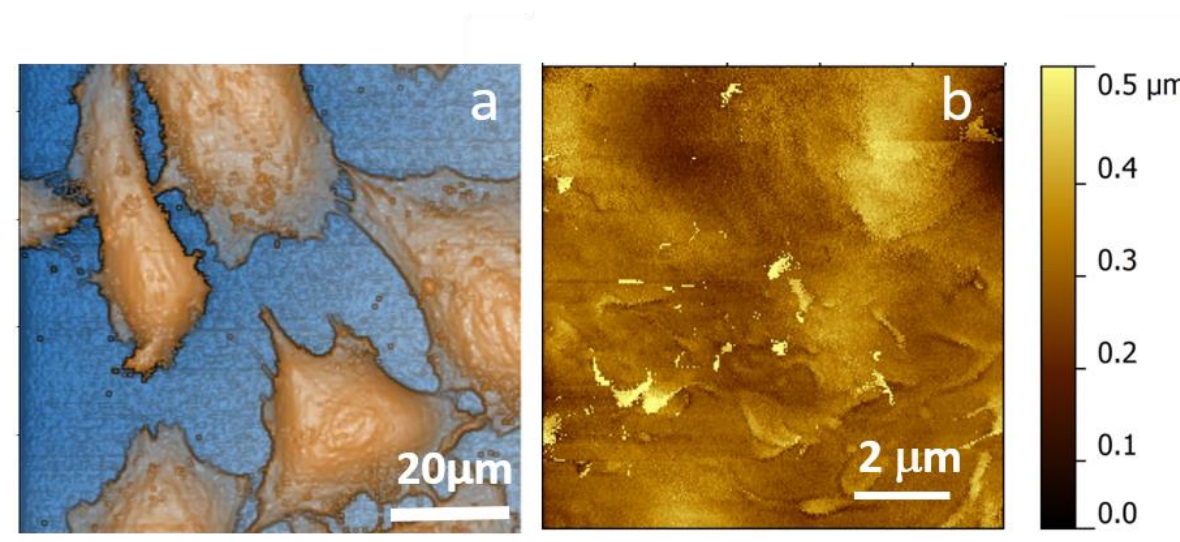

Figure 4.2 (a) The SICM topography image with the enhanced color of fixed HeLa cells without exposing to CPNs. (b) The SICM topography image of a zoom in area of a fixed HeLa cell membrane. $2^{\text {nd }}$ order flattening is applied to the image to remove cell contour.

The chemical structure of CPN is shown in the top right corner of Figure 4.3a. The backbone of CPN is hydrophobic. The side chains make the CPNs positively charged. The zeta potential of CPNs in water is measured $25.4 \pm 3.7 \mathrm{mV}$. The CPNs have also been imaged by AFM (Figure 4.3b-c) on both mica and glass substrate. We performed AFM imaging both in the air and in PBS solution and observed similar size and shape in both conditions. The height of CPN is significantly reduced in AFM topography image. The lateral size (long axis) of $\mathrm{CPN}$ is about $30-50 \mathrm{~nm}$, which is close to the mean diameter 56 $\mathrm{nm}$ measured by dynamic light scattering method. The previous study suggests these CPNs enter HeLa cells via various endocytosis pathways including caveolae-mediated endocytosis, as well as non-energy dependent entry pathways such as diffusion through the membrane. ${ }^{21}$ The high cell surface morphology changes after short time exposure of CPNs that is explained in the next paragraph can be associated with CPNs' efficient entry pathways. No toxicity was observed after incubating these CPNs with HeLa cells over 24 hours. 


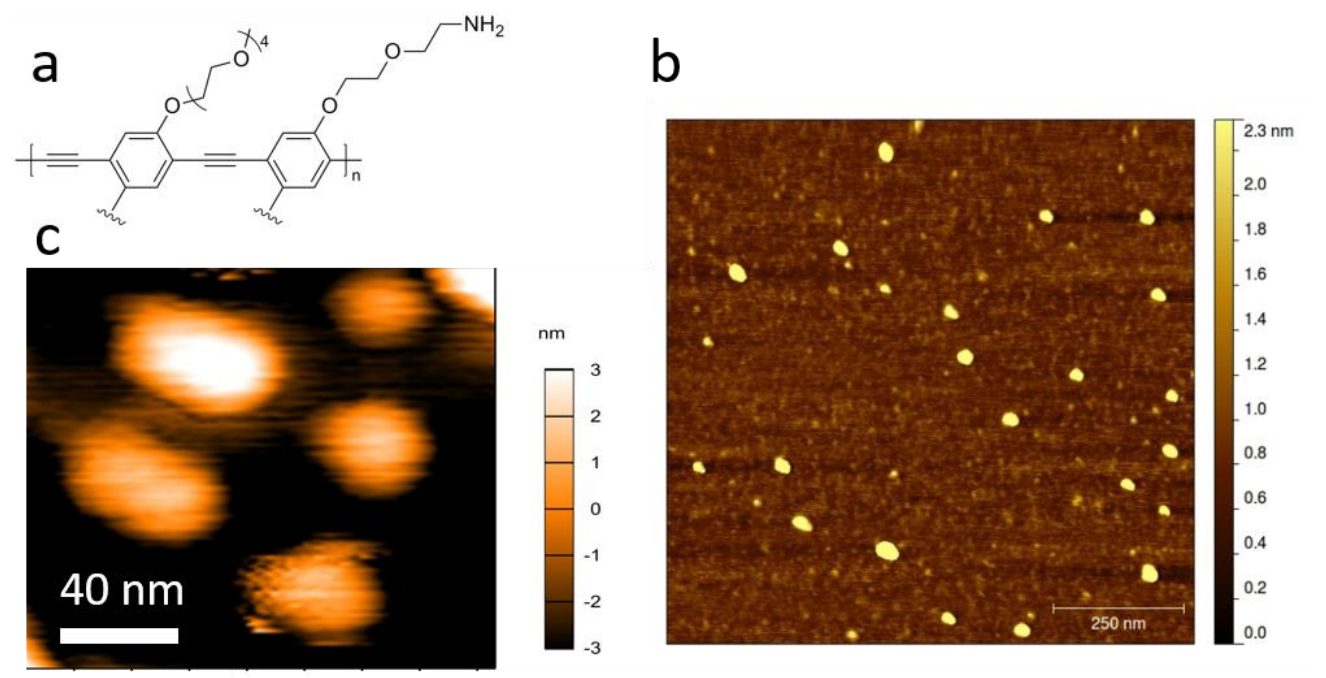

Figure 4.3: (a) The chemical structure of CPN.(b-c) AFM topography images of CPNs. The images were taken in tapping mode in air. The CPNs were immobilized on the glass substrate (zoom-in image) in (c) or mica substrate (large scale image) in (b).

The HeLa cells were incubated with $10 \mu \mathrm{M}$ CPNs for one hour. After rinsing, these HeLa cells were imaged by confocal fluorescence microscope which has explained in chapter 2 (under section 2.2.3). A typical result is shown in Figure 4.4 (b-c). Several micron sized fluorescence spots were observed and scattered around the cell surface. In addition, a large number of small fluorescence dots with the size of one or a few pixels (pixel size $60 \mathrm{~nm}$ ) were observed over the cell surface. Those large fluorescence spots are more often observed at the edge of the cell. A significant fraction of cells (about 30-40\%) also showed large fluorescence spots at the top cell surface. Figure $4.4 \mathrm{~b}$ is a single section confocal fluorescence image overlaying on a bright-field image of a typical HeLa cell, which was taken roughly at the top surface $(5 \mu \mathrm{m}$ above the cell edge). Figure $4.4 \mathrm{c}$ is a threedimensional (3D) fluorescence image of the same cell, composed of 33 confocal sectional images with a z-axis resolution $0.5 \mu \mathrm{m}$. This image showed that the large fluorescence spots distributed at the outer surface of the cell. The inset in Figure $4.4 \mathrm{~d}$ showed the zoom- 
in fluorescence image at the position indicated by the red rectangle in Figure 4.4a. Large fluorescence spots are clear. Green fluorescence in these images was emitted from the CPNs since no green fluorescence was observed in the control samples with no CPN incubations. It should also be pointed out that green fluorescence was rarely detected over the bare glass substrate regions. From the observation, it was concluded that CPNs soft aggregation nature allows efficient interaction with the hydrophobic cellular membrane.

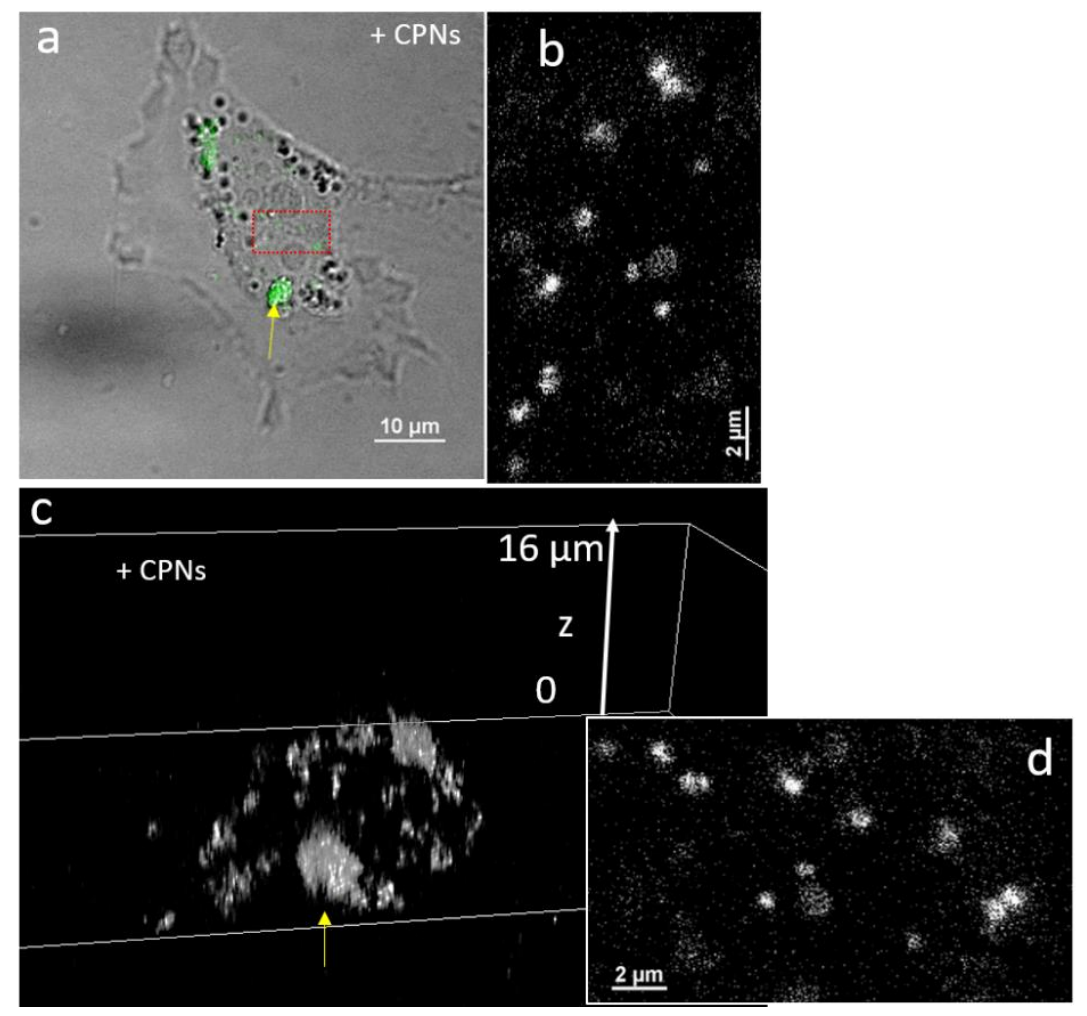

Figure 4.4: (a) The overlay of a single section confocal microscope bright field image and green channel fluorescence image at $5 \mu \mathrm{m}$ above the cell edge. The live HeLa cell was imaged after incubation with $10 \mu \mathrm{M} \mathrm{CPN}$ for 1 hour. (b) The fluorescence image of the red rectangular area in (a). (c) The 3D fluorescence image of the same cell in (a), which is composed of 33 images with $0.5 \mu \mathrm{m}$ resolution in z-axis. The yellow arrow indicates the same location in (a). (d) The fluorescence image of the red rectangular area in (a).

By considering that a typical culture medium contains high serum proteins, loosely aggregated CPNs will likely dissociate into smaller CPNs or CPN/protein aggregates. With 
the hydrophobicity of aromatic conjugated backbones, the CPN/protein adducts could still exhibit a strong interaction with the cellular membrane. It was hypothesized that highly positively charged polymers [i.e., polyethyleneimine (PEI)] would also form protein adducts; however, the interaction with the cellular membrane was likely related to the protein-cell surface receptors. Confocal microscopic images supported clearly CPNs' preferential attachment on the cell membrane. Small green spots inside the cell, which was likely from CPNs entered the cells was also observed. Large fluorescence spots were never observed inside the cells at this early stage.

A typical SICM topography image (with enhanced color) of the HeLa cells after the incubation of $10 \mu \mathrm{M}$ CPNs for one hour is shown in Figure 4.5a. Compared to the cells without CPNs treatment (Figure 4.2a-b), the surfaces of CPN treated cells became obviously rougher with the appearance of additional structures, which are likely induced by the attached CPNs as suggested by the fluorescence images.

More details of those new surface structures can be revealed in the higher resolution SICM topography images as shown in Figure 4.5b. In this figure, a large number of interconnected protrusions appeared on the cell surface. The height profile across the blue dashed line in Figure $4.5 \mathrm{~b}$ is shown in Figure 4.5f. It should be noted that SICM measurements give accurate height measurements even for the soft objects. The height of these protrusions ranged from $0.50 \mu \mathrm{m}$ to $1.20 \mu \mathrm{m}$ and the mean value was $0.87 \pm 0.16 \mu \mathrm{m}$. The lateral size of these protrusions ranged from $0.45 \mu \mathrm{m}$ to $1.30 \mu \mathrm{m}$ and the mean value was $0.81 \pm 0.15 \mu \mathrm{m}$ (Figure $4.5 \mathrm{~d}$ ). Because the size of individual CPN is below $100 \mathrm{~nm}$, the observed interconnected protrusions after CPN treatment should be attributed to combined 
cell membrane structures with CPN aggregates. CPN aggregates are likely formed at the cell membrane after the initial interaction with serum proteins.

Two downside spikes with smaller width also appeared in the height profile Figure 4.5f, which were attributed to the pits on the cell membrane. These pits (or tiny holes) were not obvious in the flattened topography image Figure $4.5 \mathrm{~b}$, but are distinguishable in the enhanced color image Figure 4.5c. For the pits, the lateral size range was $0.10-0.43 \mu \mathrm{m}$ and the mean value was $0.26 \pm 0.06 \mu \mathrm{m}$ (Figure $4.5 \mathrm{e}$ ). The attachment of CPNs to the cell surface is the first step for CPN endocytosis. After the attachment, some CPNs enter the cell through endocytosis. Using biochemistry methods, CPNs enter the HeLa cells using various endocytosis pathways including caveolae-mediated endocytosis was confirmed. Previous SICM studies also observed endocytic pits. ${ }^{24}$ The observed pits here can be related to caveolae-mediated endocytosis. The smaller size of pit suggested that CPN might enter the cell as a small cluster. The relatively large CPN clusters likely adhere to the cell membrane, and uptake processes of these large CPN aggregates are much slower than the small aggregates as further dissociation/de-aggregation processes required. 
a
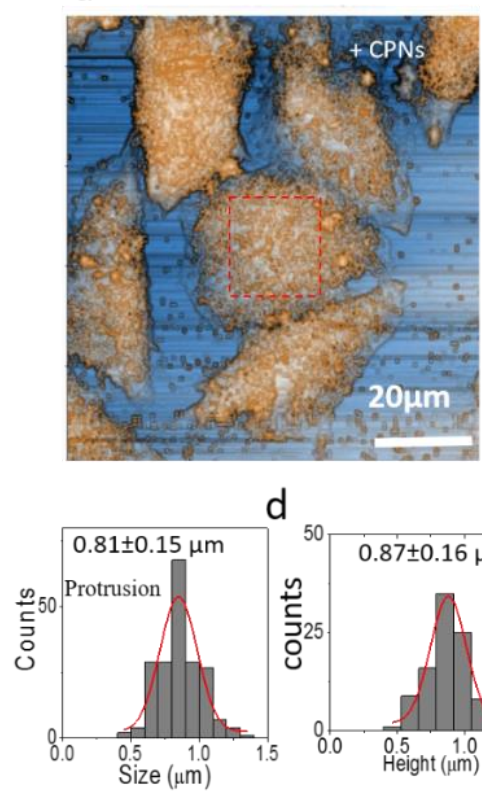

b

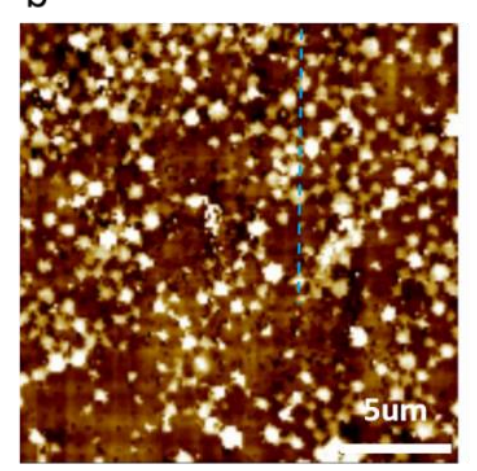

e

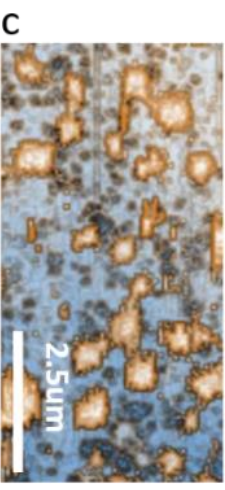

$f$
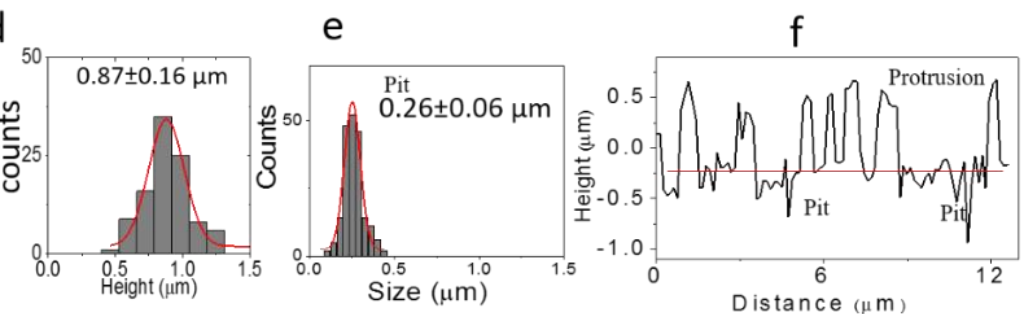

Figure 4.5 (a) The SICM topography image with the enhanced color of HeLa cells treated with $10 \mu \mathrm{M} \mathrm{CPN}$ for $1 \mathrm{~h}$. (b) A higher resolution image of the red rectangular area in Figure 3.5a. $2^{\text {nd }}$ order flattening was applied to the image to remove cell contour. (c) A highresolution SICM topography image with the enhanced color of a small area of the cell surface. (d-e) The histograms of protrusion size and height (d), and pit size (e). The histograms are fitted by Gaussian functions. (f) The height profile across the blue dash line in Figure b. All the cells were fixed in $4 \%$ paraformaldehyde.

CPNs remained strongly on the cell membrane after stringent washing before imaging, while few CPNs were observed on the glass substrate. As a control experiment, the cell surface morphology change after one-hour of incubation with one-micron diameter solid polystyrene (PS) beads was also studied. Although PS exhibits high hydrophobicity, only a few isolated one-micron size (both height and diameter) protrusions on the cell surface were observed, and they were attributed to individual beads based on their size. One typical image of the polystyrene beads is shown in Figure 4.6. No pits were identified. The small number of attached polystyrene beads on the cell surface (even with longer incubation time) suggests that individual polystyrene beads interact weakly with HeLa cells 
surface. This observation confirms that the softness and lose aggregation nature of CPNs are important factors to enhance $\mathrm{CPN}$ cellular interaction.

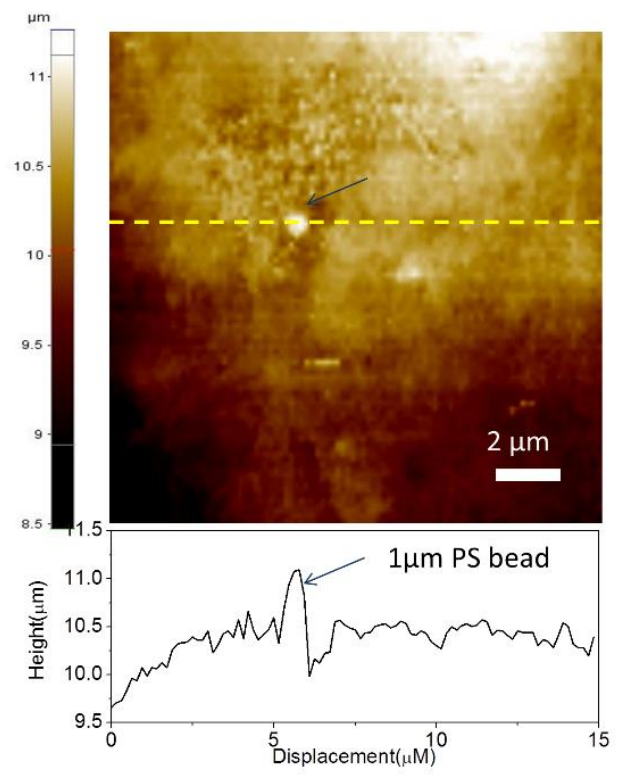

Figure 4.6 SICM topography image and height profile across the dashed line. One $1 \mu \mathrm{m}$ size polysterene (PS) bead with $1 \mu \mathrm{m}$ size can be identified (indicated by the arrow).

It is well known that the charge and hydrophobicity of polymer NPs play important roles in endocytosis process. The positive charge and hydrophobicity of CPNs should facilitate the aggregates of CPNs at the negatively charged cell surface through electrostatic and hydrophobic interactions. Adsorbed CPNs on the cell surface can lead to a higher endocytosis efficiency. To examine the role of the hydrophobicity on cellular interaction, the cell surface roughness of HeLa cells treated with branched PEI under the same experimental condition we monitored. PEI is widely used for drug delivery and has a positive charge at $\mathrm{pH} 7 .{ }^{25}$ The hydrophobicity of branched PEI can be negligible compared to CPNs. To quantitatively determine and compare cell surface roughness induced by CPN and PEI, respectively, a surface roughness parameter called surface area ratio. Surface area ratio here is defined as Surface Area Ratio $=100 \% \times($ Surface Area - Geometric Area $) /$ 
(Geometric Area) was used. The geometric area is the projected area of a rough surface on a flat surface. The detail definitions of surface area and geometric area are given in chapter 2 under section 2.2.5 (Figure 2.5). This ratio is used to quantify the surface roughness level. Zero means the surface is perfectly smooth and higher value means the surface roughness level is higher. The surface area ratio can reflect the area density, size, and shape of these extra features. In the analysis, the cell membrane area with large blebs (size $>2 \mu \mathrm{m}$ ) is excluded.

As shown in Figure 4.7a, the surface area ratios of nearly all (94\%) control cells are below 30 and the mean value is about 15 . After one hour CPN treatment, a broad distribution of the surface area ratios and a significant fraction of cells (36\%) showed high surface area ratios was noticed (see Figure 4.7b). This heterogeneity was also observed by confocal fluorescence microscopy. No uniform fluorescent signals were observed from all cells. Although the further detailed investigation is needed, it was speculated that the heterogeneity on both fluorescent and SICM topography images might be associated with cell cycles. It is difficult to differentiate the protrusions induced by CPN aggregates and the original features of cell membrane for cells with surface area ratio below 30 . Therefore only the data with surface area ratio above 30 was used. The mean value of cell surface area ratio increased to 52 after one hour CPN treatment. In contrast, there is no obvious change in the surface area ratio distributions of PEI treated cells compared to untreated control cells, as shown in Figure 4.7c. 


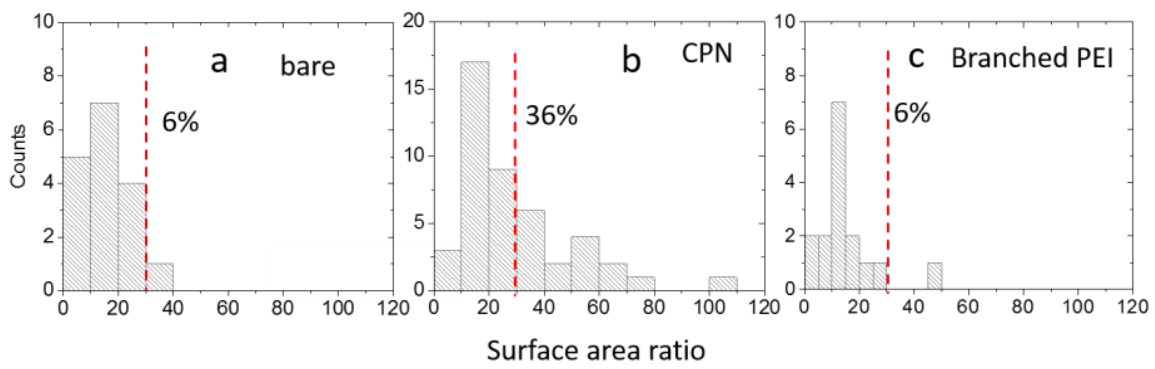

Figure 4.7 The distribution of SICM measured surface area ratio value of HeLa cells without (a) and with CPN (b), and with PEI (c) incubation at the same condition $(10 \mu \mathrm{M}$ for $1 h)$.

The high-resolution topography image of a typical PEI treated surface is shown in Figure 4.8. The PEI treated cell surface normally showed more features than bare cell surface, suggesting attachments of PEI to the cell surface. However, these features are too small to alter the value of surface area ratio significantly. No large clusters at the cell surface were observed due to the well-solvated hydrophilic PEI. Pits on the cell surface were also not observed.
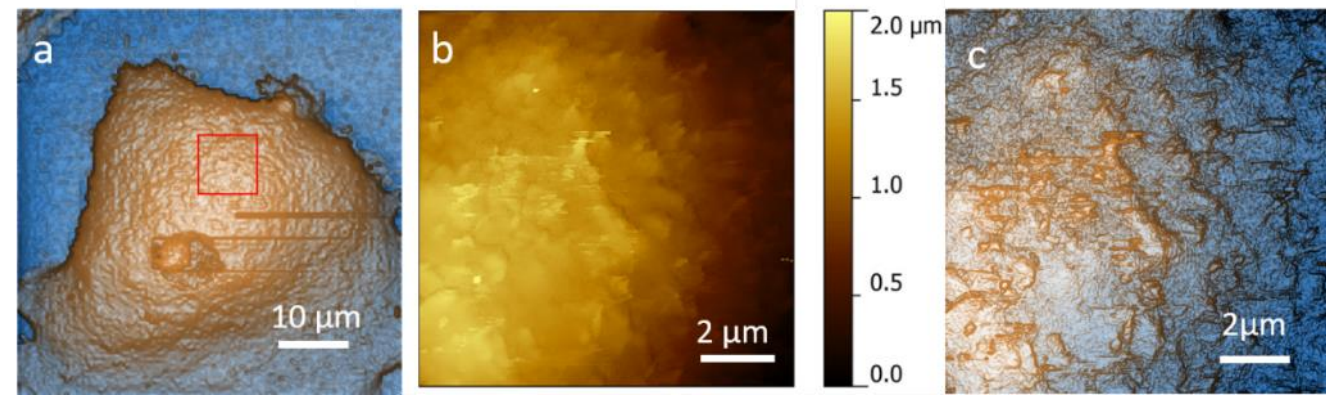

Figure 4.8 SICM images of a typical PEI treated HeLa Cells. (A) Low resolution (312nm/pixel) SICM enhanced color topography image. (B) High resolution (39 nm/pixel) SICM topography image. (C) Enhanced color image of (B). The cells were treated by 10 $\mu \mathrm{M}$ PEI for 1 hour and were fixed before imaging.

Fluorescent microscopic images of cells pretreated with actin inhibitors or metabolism inhibitors, respectively, are very similar to those of cells without any pretreatments. The actin inhibitor prevents the involvement of cell skeleton movement 
during CPN endocytosis. The metabolism inhibitor also prevents energy-dependent cell activities associated with CPNs. These observations suggest that the cellular adsorption of CPNs is driven by diffusion followed by hydrophobic adsorption. In addition, the subsequent initial endocytosis processes are very slow or not related to cellular activities require energy. We also checked CPN attachment to fixed cells with paraformaldehyde. The paraformaldehyde treatment denatures proteins and enzymes by crosslinking to terminate biochemical activities of live cells. CPNs were not observed at the cell surface under the same incubation condition (i.e., $1 \mathrm{~h}$ at $10 \mu \mathrm{M}$ CPNs). From this observation, we believe that CPN adsorption is somehow associated with live cell activities.

Cell surface morphology change was monitored as a function of incubation time at a $\mathrm{CPN}$ concentration $(10 \mathrm{mM})$. The surface area ratio reached the maximum value after $1 \mathrm{~h}$ incubation and then decreased to the initial value (Figure 4.10a). A similar trend was observed from the confocal fluorescence microscopic study. We imaged the cells after incubation with $10 \mu \mathrm{M}$ CPNs for 1, 2.5 and 4 hours. The total green fluorescence area on the cell membrane became much smaller after an increased incubation time (Figure 4.4 and 4.9). 


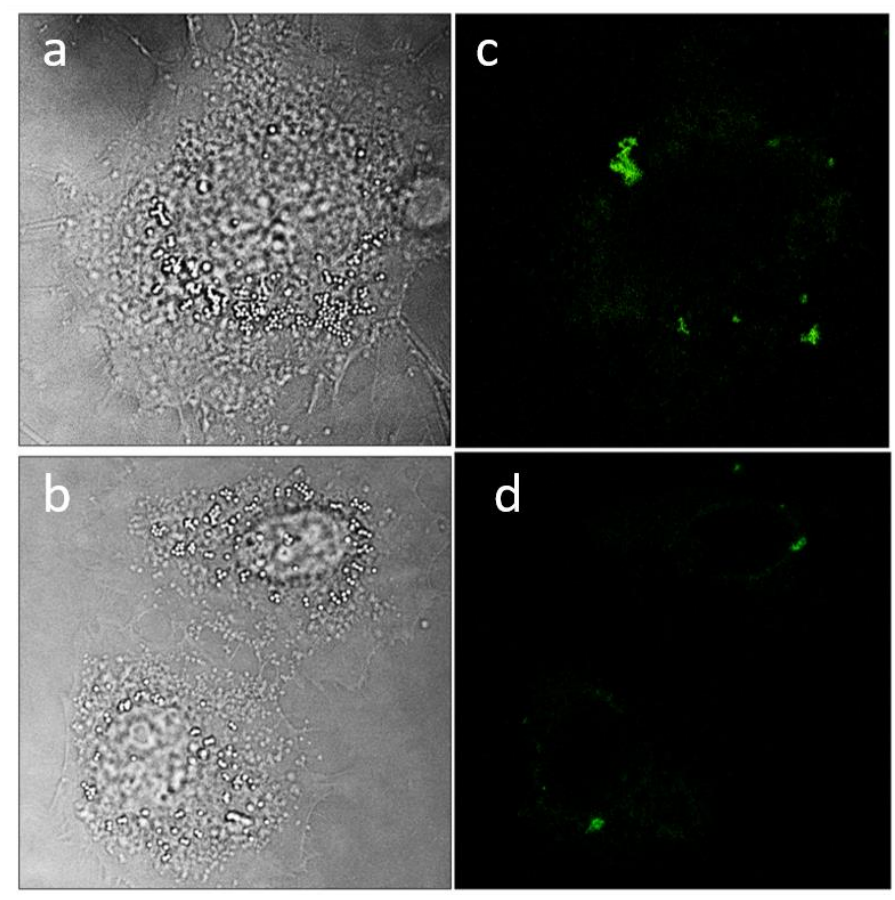

Figure 4.9 Confocal microscope bright field overlay images (a-b) and 3D fluorescence images (c-d) to show three HeLa cells after $4 \mathrm{~h}$ CPN incubation.

The attachment of CPNs starts with mass transport (diffusion and fluid movement) in the culture medium. This step is only controlled by extracellular environment conditions. After landing on the cell surface, the CPNs interact with the cell surface and other neighboring CPNs, mainly governed by electrostatic and hydrophobic interactions as we discussed previously. If the adsorption of CPNs at the cell surface is only controlled by physical interactions, the surface area ratio should be a constant after reaching the maximum value (the equilibrium state) because the CPNs are always abundant in the solution. The time-dependent behaviors indicate that CPN attachment and following internalization are related to the cellular activities. After internalizing a large number of CPNs, the cells may gradually lower their activities in retaining the CPNs at the cell surface. As shown in Figure 4.10b, the maximum surface area ratio increases as the 
concentration of CPNs increase. The increase in maximum surface area ratio can be attributed to the enhanced mass transport of CPNs to the cell surface. Interestingly, the time to reach maximum surface area ratio also increases with CPNs concentration in the culture medium, as shown in Figure 4.10c. If no cell activity is involved, we should expect a decrease in time. The result is because the rate of collision events between free CPNs in solution and the cell surface is proportional to CPN concentration. Therefore, the same number of CPNs will take a shorter time to reach the cell surface when CPN concentration in the solution is higher. However, the cell may be reluctant to retain and internalize all the CPNs collide with the cell surface. These time and concentration dependence results suggest that the cell morphology change is controlled both by physical interactions and cell activities. The competition between CPN physical attachment and cell activities affects the observed cell surface morphology and roughness.
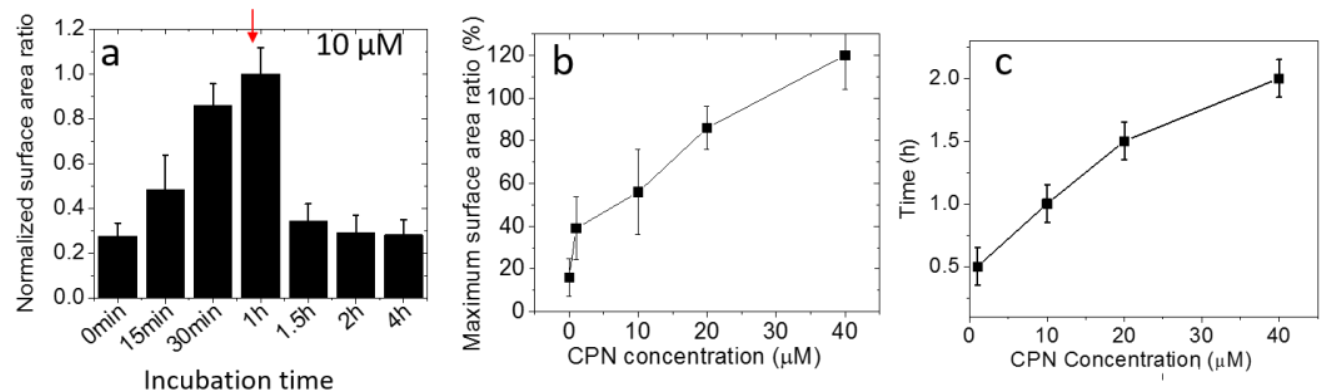

Figure 4.10 (a) The incubation time dependence of cell surface area ratio for HeLa cell coculture with $10 \mu \mathrm{M}$ CPNs. The surface area ratio magnitude was normalized by the maximum surface area ratio, which is indicated by the red arrow at $1 \mathrm{~h}$. Due to the large variation of surface area ratio, we only used data points above 30 for the mean value for time 15 minutes, 30 minutes and $1 \mathrm{~h}$. There are not enough data points above 30 for time $0,1.5 \mathrm{~h}, 2 \mathrm{~h}$ and $4 \mathrm{~h}$, we still use all the data points for the mean value. (b) The maximum surface area ratio as a function of CPN concentration. (c) The time to reach maximum surface area ratio versus $\mathrm{CPN}$ concentration. The value is Mean $\pm \mathrm{SD}$ (standard deviation) in (b) and (c). 


\section{4: Conclusion}

In summary, we have demonstrated that the capability of SICM in the detailed study of the attachment of cationic CPNs to the cell surface, corresponding morphology change of the cell surface, and the effect of the following endocytosis. SICM and confocal fluorescence images revealed that $\mathrm{CPNs}$ are in cluster form at the cell surface before entering the cells. These clusters enhance the attachments of CPNs to the cell surface. The physicochemical properties (hydrophobicity and positive charge) of polymer CPNs also affect the attachments and aggregations of CPNs to the cell surface. After CPN attachment, the cells actively internalize CPNs. The CPN attachment is the first step of CPN endocytosis. Therefore, the careful design of the physicochemical properties of CPN to promote cellular attachment can significantly impact the CPN endocytosis efficiency.

\section{5: References}

1. Shan, Y., Panday, N., Myoung, Y., Twomey, M., Wang, X., Li, W., Celik, E., Moy, V., Wang, H., Moon, J.H. \& He, J. Macromolecular Bioscience 2016, 16, 599.

2. Canton, I. \& Battaglia, G. Chem. Soc. Rev. 2012, 41, 2718.

3. Brandenberger, C., Mühlfeld, C., Ali, Z., Lenz, A.-G., Schmid, O., Parak, W.J., Gehr, P. \& Rothen-Rutishauser, B. Small 2010, 6, 1669.

4. $\quad$ Lu, F., Wu, S.-H., Hung, Y. \& Mou, C.-Y. Small 2009, 5, 1408.

5. Seifert, J., Rheinlaender, J., Novak, P., Korchev, Y.E. \& Schäffer, T.E. Langmuir 2015, 31, 6807 .

6. Rheinlaender, J., Geisse, N.A., Proksch, R. \& Schäffer, T.E. Langmuir 2011, 27, 697.

7. Hansma, P., Drake, B., Marti, O., Gould, S. \& Prater, C. Science 1989, 243, 641. 
8. Prater, C.B., Hansma, P.K., Tortonese, M. \& Quate, C.F. Review of Scientific Instruments 1991, 62, 2634.

9. Miragoli, M., Moshkov, A., Novak, P., Shevchuk, A., Nikolaev, V.O., ElHamamsy, I., Potter, C.M.F., Wright, P., Kadir, S.H.S.A., Lyon, A.R., Mitchell, J.A., Chester, A.H., Klenerman, D., Lab, M.J., Korchev, Y.E., Harding, S.E. \& Gorelik, J. J.R.Soc., Interface 2011, 8, 913.

10. Shevchuk, A.I., Hobson, P., Lab, M.J., Klenerman, D., Krauzewicz, N. \& Korchev, Y.E. Biophysical journal 2008, 94, 4089.

11. Shin, W. \& Gillis, K.D. Biophysical journal 2006, 91, L63.

12. Gorelik, J., Shevchuk, A.I., Frolenkov, G.I., Diakonov, I.A., Lab, M.J., Kros, C.J., Richardson, G.P., Vodyanoy, I., Edwards, C.R.W., Klenerman, D. \& Korchev, Y.E. Proc. Natl. Acad. Sci. U.S.A. 2003, 100, 5819.

13. Gorelik, J., Zhang, Y., Shevchuk, A.I., Frolenkov, G.I., Sánchez, D., Lab, M.J., Vodyanoy, I., Edwards, C.R.W., Klenerman, D. \& Korchev, Y.E. Mol. Cell. Endocrinol. 2004, 217, 101.

14. Ying, L., Bruckbauer, A., Zhou, D., Gorelik, J., Shevchuk, A., Lab, M., Korchev, Y. \& Klenerman, D. Physical Chemistry Chemical Physics 2005, 7, 2859.

15. Shevchuk, A.I., Frolenkov, G.I., Sánchez, D., James, P.S., Freedman, N., Lab, M.J., Jones, R., Klenerman, D. \& Korchev, Y.E. Angewandte Chemie International Edition 2006, 45, 2212.

16. Tuncel, D. \& Demir, H.V. Nanoscale 2010, 2, 484.

17. Ko, Y.-J., Mendez, E. \& Moon, J.H. Macromolecules 2011, 44, 5527

18. Moon, J.H., McDaniel, W., MacLean, P. \& Hancock, L.F. Angewandte Chemie International Edition 2007, 46, 8223.

19. Silva, A., Nguyen, A., Ye, C., Verchot, J. \& Moon, J. BMC Plant Biology 2010, 10, 291.

20. Wang, X., Kim, Y.-G., Drew, C., Ku, B.-C., Kumar, J. \& Samuelson, L.A. Nano Letters 2004, 4, 331.

21. Lee, J., Twomey, M., Machado, C., Gomez, G., Doshi, M., Gesquiere, A.J. \& Moon, J.H. Macromol. Biosci. 2013, 13, 913.

22. Mendez, E. \& Moon, J.H. Chem. Commun. 2013, 49, 6048. 
23. Dausend, J., Musyanovych, A., Dass, M., Walther, P., Schrezenmeier, H., Landfester, K. \& Mailänder, V. Macromolecular Bioscience 2008, 8, 1135.

24. Shevchuk, A.I., Novak, P., Taylor, M., Diakonov, I.A., Ziyadeh-Isleem, A., Bitoun, M., Guicheney, P., Lab, M.J., Gorelik, J., Merrifield, C.J., Klenerman, D. \& Korchev, Y.E. The Journal of Cell Biology 2012, 197, 499.

25. Breunig, M., Lungwitz, U., Liebl, R. \& Goepferich, A. Proceedings of the National Academy of Sciences of the United States of America 2007, 104, 14454. 


\section{CHAPTER 5: SIMULTANEOUS IONIC CURRENT AND POTENTIAL DETECTION OF NANOPARTICLES BY A MULTIFUNCTIONAL NANOPIPETTE}

In chapter 4 , we have demonstrated the capability SICM for the study of morphological changes in the cell membranes during endocytosis process by acquiring and analyzing the topography images. In addition to the topography images, this dissertation aims to develop the SICM for the study of topography and extracellular potential distributions of the single living cell at the same time. For the simultaneous detection of the topography and the potential distributions, we have used the double barrel theta nanopipette as a probe but the sensitivity of the potential detection was pretty low. As we know a good probe is always very important for the scanning probe methods. Hence, to enhance the sensitivity of the potential measurement, we fabricated and characterized the multifunctional nanopipette. This Chapter presents the fabrication and characterization of the multifunctional nanopipette and its ability for the simultaneous detection of ion current and potential change during the translocation of charged gold nanoparticles through the nanopore. The majority of the content of this chapter has been adapted from my research work published in a peer-reviewed paper. ${ }^{1}$

\section{1: Introduction}

Nanopore-based single molecule/nanoparticle analytical methods have made significant progress in the last two decades. To improve the sensitivity and selectivity of the nanopore sensing methods and to add new functionality to nanopore devices, it is desirable to detect single entity translocation events using simultaneous multimode detection methods. In recent years, several nanopore-based single entity multimode detection methods have been demonstrated. For example, electrical potential and ionic 
current change were detected when individual DNA molecules translocated through the nanopore of a nanopore-nanowire sensor. ${ }^{2}$ Both fluorescence and ionic current changes were detected when single dye molecule flows through a carbon nanotube nanopore. ${ }^{3}$ Single molecule fluorescence and ionic current changes were detected for fluorescence molecule tagged DNA and DNA-protein complexes, using nanopore/zero mode waveguide device. ${ }^{4}$ Surface-enhanced Raman scattering (SERS) signal and the ionic current was simultaneously detected when single gold nanoparticle transported through gold coated nanopore. $^{5}$

As a subgroup of nanopore, nanopipette is modeled as a conical shaped nanopore and has been used for single DNA, protein, and nanoparticle (NP) analysis. ${ }^{6-9}$ Compared with other solid-state nanopore, one important advantage of nanopipette is that it can be made cheaply and reproducibly with a few tens of nanometer resolution from glass or quartz capillary tubes. In addition, it is highly versatile in application and fabrication. For example, we can directly use the nanopipette as a nanopore sensor for chemical and biological sensing and electrophysiological applications. Owning to its tip geometry, nanopipette is also developed as a scanning probe for scanning ion conductance microscopy (SICM) and scanning electrochemical microscopy (SECM). ${ }^{10}$ The fabrication of multifunctional nanopipette, with both nanopore and nanoelectrode functions at the nanopipette tip, has attracted many interests recently. One motivation is to achieve multimode detection for single molecule and nanoparticle. ${ }^{11}$ The other is to integrate SICM and SECM for a hybrid scanning probe microscopy technique that is powerful in single live cell analysis and imaging. ${ }^{12-14}$ The fabrication method for nanopore/nanoelectrode multifunctional nanopipette is simple and versatile. Several methods have been developed, 
and they are generally of two types. The first type is based on double-barrel nanopipettes made from theta micropipettes. ${ }^{15}$ One barrel is converted to a nanoelectrode by filling the barrel with conductive materials, such as pyrolytic carbon through the pyrolysis process. ${ }^{12}$ The other barrel remains open for ionic current measurement. The second type is to deposit a conductive thin film on the outside or inner wall of a single barrel nanopipette. ${ }^{7,16}$ The single barrel remains open as the fluidic channel. The conductive thin layer is used as the electrode and often needs to be partially coated with Parylene, ${ }^{17}$ or electrophoretic paint, ${ }^{12}$, ${ }^{18}$ for insulating and for improving its mechanical, chemical and electrochemical stability. We adapted the first type of methods to fabricate multifunctional nanopipettes with both nanopore and nanoelectrode functions from quartz theta pipettes. The carbon nanoelectrode (CNE) and nanopore are very close to each other at the nanopipette tip. The CNE also extends out of the tip slightly.

So far, the CNEs of the multifunctional nanopipettes were always used for electrochemical current measurements. The nanopore and nanoelectrode of the multifunctional nanopipette have not been used for multimode NP analysis, especially noncatalytic NPs. In the present work, we demonstrated that we could use these nanopipettes for simultaneous potential and ionic current sensing on the translocation of NPs. As shown in the experimental setup scheme of Figure 2.8, a voltage meter with a high input impedance is connected to the CNE for potential measurement, and a low-noise current amplifier is connected to the nanopore for ionic current measurement. Here we used $40 \mathrm{~nm}$ diameter citrate stabilized negatively charged gold nanoparticles (GNPs). Because of the screening effect of the ions, it is technically challenging to detect the movement of a labelfree GNP in an electrolyte based on charge using an electrical method. This setup utilizes 
the advantages of both nanopore and CNE. It offers the opportunity to detect the charged GNPs (based on their surface charge/surface potential) when they approach, accumulate and translocate through the nanopore. The nanopore captures the nearby moving GNPs more efficiently by the enhanced local electric field and brings them to the proximity of the CNE. The distance between GNPs and CNE is so small that the CNE can work as an in situ potential probes. The protrusion of $\mathrm{CNE}$ out of the nanopore entrance helps the CNE to sense several GNPs at the same time and detect the GNPs further away from the nanopore orifice. In this way, the potential results shed light on the accumulation and dynamic cluster assembly of GNPs outside the nanopore, which is difficult to be detected at this size scale. In addition, the nanopore helps to detect one GNP out from several GNPs in a cluster. We are therefore able to acquire both the potential and the current changes induced by a single GNP during its translocation through the nanopore. We also carried out finite element method (FEM) simulations to understand the potential detection mechanism. In general, a potential probe can directly detect the surface potential/surface charge of an entity although the potential detection range is very short in an ionic solution. The nanowire-nanopore work reported by Xie et al. ${ }^{2}$ suggested a new potential sensing mechanism that is unique for nanopore devices. The translocation of a charged entity through a nanopore alters the ionic resistances of the ionic circuit, leading to potential redistributions both outside and inside the nanopore-based on the voltage divider rule. We have found that the potential sensing mechanism in this nanopore/CNE system is a combination of both mechanisms, owning to the tip geometry of the nanopipette and the low ionic strength of the solution. It is very different from the nanowire-nanopore work. The fabrication of multifunctional nanopipettes is fast and easy, with the price of relatively 
large geometry fluctuations. However, the potential sensing mechanism is robust despite the large variations in the nanopore and CNE geometry.

\section{2: Research and Discussion}

\subsection{1: Multifunctional Nanopipette Fabrication and Characterization}

The multifunctional nanopipettes were fabricated from quartz theta capillary tubes. The details of dual-nanopore nanopipette fabrication are given in the chapter 2 in Section 2.2.1.2. The fabricated dual-nanopore nanopipettes were imaged by an optical microscope and a scanning electron microscope (SEM) which are shown in Figure 5.1a. In the top view image (Figure 5.1a right inset), a separation between two similar sized nanopores $(\sim 60 \mathrm{~nm})$ can be barely resolved. The nanopore size was within the range of reported values using the same pulling parameters. ${ }^{19}$ From five samples, the mean outer half cone angle $\theta=6.5$ $\pm 0.5^{\circ}$ was obtained, which was used to estimate the nanopore size using following equation 5.1. It should be noted that the inner half cone angle may be smaller than the outer angle, as revealed by TEM images. ${ }^{20}$ Therefore, we may systematically underestimate the nanopore size. Therefore Equation 5.1 only gives a crude estimation of the nanopore size.

As reported previously, ${ }^{8,21}$ the surface charge effect of a conical shaped nanopore can be ignored at the small bias range (i.e., $\mathrm{V}<\mathrm{k}_{\mathrm{B}} \mathrm{T} / \mathrm{e}$, where $\mathrm{k}_{\mathrm{B}}$ is the Boltzmann constant, $\mathrm{T}$ is the temperature, and $\mathrm{e}$ is the elementary electron charge). A simple analytical equation shown below was used for nanopore estimation:

$\mathrm{D}=\frac{4}{\mathrm{kR}_{\mathrm{p}}}\left(\frac{1}{\pi \tan \theta}\right)$ 
$\mathrm{Rp}$ is the nanopore resistance, and $\kappa$ is the conductivity of the electrolyte. The $\mathrm{Rp}$ was determined from the IV measurements at $10 \mathrm{mM}$ PBS. The $\kappa$ for $10 \mathrm{mM}$ PBS (at $\mathrm{pH}$ 7.4) was determined to be $1312 \mu \mathrm{S} / \mathrm{cm}$ from conductivity measurements.

In the next step, one barrel of the dual-nanopore nanopipette was filled with pyrolytic carbon to form carbon nanoelectrode (CNE) at the tip. ${ }^{13}$ The details of CNE fabrication are given in chapter section 2.3.1. The final CNE geometry can be controlled by the flow speed/pressure of butane (carbon source) and argon (protective gas) during carbon deposition. After CNE fabrication, the nanopipette was imaged again by an optical microscope (see figure 2.4b in chapter 2 under section 2.2.2.3) and the SEM (see Figure 5.1b). The solid black color of one barrel of the nanopipette suggests the successful fabrication of the CNE. The cross-section SEM image of a broken tip (Figure 5.1c) further confirms that the pyrolytic carbon has fully filled one barrel of the nanopipette. Figure 5.1b shows the SEM image of a representative nanopore/CNE nanopipette tip. A small section of the CNE protrudes out of the tip. The diameter of the protruded CNE, which was around $70 \mathrm{~nm}$ for this one, was typically bigger than the nanopore size, which is around 50 nm. 

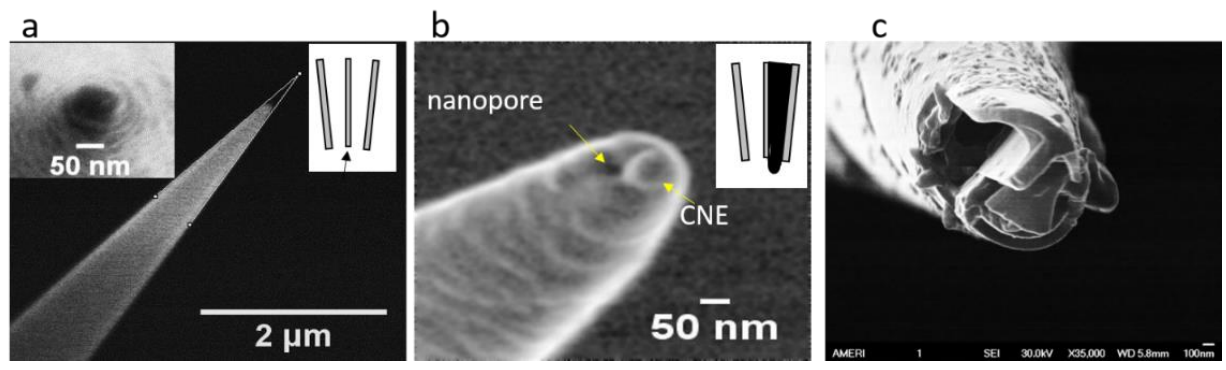

Figure 5.1. (a)The SEM image of the side view of a dual-nanopore nanopipette. The conical angle is measured using the two white guidelines. The left inset of (a) shows the top view of the dual-nanopore nanopipette and two nanopores can be barely resolved. The top right inset of (a) shows the schematic of the dual-nanopore nanopipette where the black arrow indicates the thin layer separating two separate barrel. (b) The SEM image of the tip region of the nanopore/CNE nanopipette. Both nanopore and CNE can be resolved which has pointed by yellow arrows. The top right inset of (b) shows the schematic of the nanopore/CNE nanopipette. (c)The cross-section SEM image of a broken nanopore/CNE nanopipette. Before SEM imaging, the nanopipette was coated with a gold thin film (about $5 \mathrm{~nm}$ thick) to reduce charging during SEM imaging.

In experiments, the inner diameter of the nanopore, and the surface charge of inner nanopore surface was estimated, based on current-voltage (I-V) curves (see Figure 5.2a). As demonstrated previously by both experiments and simulations, ${ }^{8,21,22}$ the surface charge effect was ignored to the I-V curve at low bias, and a simple analytical equation (equation $5.1 \mathrm{in}$ ) was used. The calculation of nanopore size is based on the ionic resistance $\mathrm{R}_{\mathrm{p}}$, which is derived from the reciprocal of the slope of the linear portion of the I-V curve within a small applied bias range (see Figure 5.2b). ${ }^{21}$ Similar $R_{p}$ for the two nanopores of the same nanopipette was always obtained, verifying that the size of two nanopores was very close. This was also consistent with other reports. ${ }^{13}$ The $\mathrm{R}_{\mathrm{p}}$ histogram from 28 dual-nanopore nanopipettes is displayed in Figure 5.2c (green color), and an average $R_{p}$ of $2.22 \pm 0.53 \mathrm{G} \Omega$ is obtained. Using half-cone angle $\theta=6.5^{\circ},{ }^{21}$ the derived average inner pore diameter is 77 $\pm 18 \mathrm{~nm}$ if assuming a circular nanopore shape. The distribution of $R_{p}$ reflects the pipetteto-pipette geometry changes under the same fabrication parameters. After CNE fabrication, 
the mean value of $R_{p}$ is increased to $3.00 \pm 1.16 \mathrm{G} \Omega$ (see the gray color histogram in Figure $5.2 \mathrm{c})$, and the corresponding diameter of the nanopore is reduced to $57 \pm 22 \mathrm{~nm}$. Therefore, the nanopore size of a nanopipette is generally reduced after the CNE fabrication step. This size reduction is attributed to two reasons, the shrinking of nanopore size after hightemperature treatment and the over-deposition of carbon at the nanopipette tip surface.

The asymmetry of IV curves from nanopipette nanopores is mainly determined by the conical geometry and the surface charge, instead of the electroosmotic flow. ${ }^{21-23}$ Assuming a fixed half-cone angle, the asymmetry of an IV can be used as a measure of surface charge density of the nanopipette, which is quantified by the rectification ratio as $r=\log \left|\frac{I_{+}}{I_{-}}\right| \cdot{ }^{24}$ A histogram of the rectification ratio of 28 nanopipettes at $\pm 0.4 \mathrm{~V}$ is shown in Figure 5.2d (green color) and $r=-0.78 \pm 0.12$. The negative sign indicates the negative surface charges at the quartz nanopipette surface. After the fabrication of CNE, the magnitude of rectification ratio $r$ is reduced to $0.66 \pm 0.26$ (Figure $5.2 \mathrm{~d}$ gray color histogram), suggesting a reduced surface charge density. This is likely due to the excess carbon at the nanopore inner surface near the tip after carbon deposition. A similar effect was observed for nanopipette nanopores after gold deposition. ${ }^{7}$ 

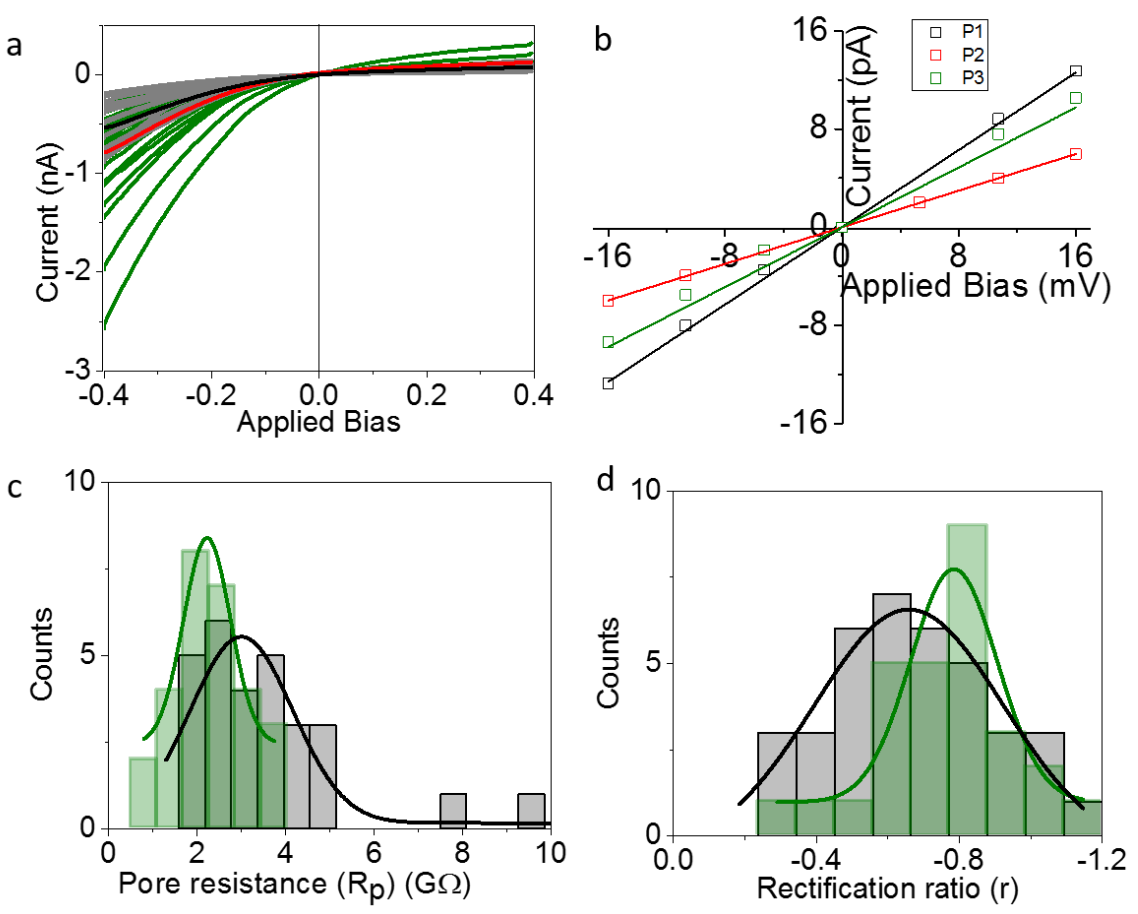

Figure 5.2. (a) IV curves of 28 nanopipettes before (green color) and after CNE fabrication (gray color). The IV curves were measured in $10 \mathrm{mM}$ PBS. The average curves are overlaid as bold red (before) and black (after) curves. (b) IVs in a small bias range for multifunctional nanopipettes P1, P2 and P3 used in the experiment. IVs were recorded in $10 \mathrm{mM}$ PBS ( $\mathrm{pH}$ 7.4). The square symbol represents the experimental data and the straight lines are fitted lines. The black, red and green curves represent IVs for P1, P2 and P3 respectively. (c) The histograms of measured pore resistance (base on IV curves) before (green color) and after CNE fabrication (gray color). The solid lines are Gaussian fits to the histograms. The mean value is $2.22 \pm 0.53 \mathrm{G} \Omega$ before $\mathrm{CNE}$ fabrication and $3.00 \pm 1.16$ G $\Omega$ after GNE fabrication. The error bar is the standard deviation of the Gaussian fits. (d) The histograms of rectification ratio $r$ before (green color) and after CNE fabrication (gray color). ). The solid lines are Gaussian fits to the histograms. The mean value is $-0.78 \pm 0.12$ before CNE fabrication and $-0.66 \pm 0.26$ after GNE fabrication. The error bar is the standard deviation of the Gaussian fits.

The CVs of these CNEs always showed the typical sigmoidal shape from ultrasmall electrodes (see Figure 2.7 under section 2.3.2), confirming the conductive CNEs were made by pyrolytic carbon instead of amorphous carbon. The effective electrode area of the CNE is determined based on the diffusion-limited current $i_{d}$ from these CVs (see Chapter 2 in section 2.3.2). As shown in Figure 5.3b, there is a big variation in the effective 
surface area of the fabricated CNEs. This is attributed to the higher carbon source (Butane gas) pressure used during CNE fabrication in order to get the protruded CNE. Without the confinement from the quartz wall, the protruded section of the CNE can grow oversized easily and quickly. The peak of the measured CNE effective electrode area distribution is around $0.3 \mu \mathrm{m}^{2}$. Using this electrode area, we estimated the CNE diameter to be $220 \mathrm{~nm}$, if assuming a cylinder geometry with the CNE length three times the radius. This estimated CNE diameter is more than four times bigger than the typical nanopore size. In addition to the overgrowth of CNE, excess carbon may be deposited on the outer and inner surfaces around the nanopipette tip to enlarge the overall CNE size.

Because the fabrication is quick and easy, NP analysis has carried out using a large number of multifunctional nanopipettes. Similar potential changes were always observed during the GNP translocation events despite the large variations in the CNE size and geometry. Herein, the data from three representative nanopore/CNE nanopipettes P1, P2 and P3 have shown. Based on the IV measurements of nanopores (after carbon deposition, Figure 5.2b) and CV measurements of CNEs (Figure 5.3a), the nanopore diameter was determined to be $85 \pm 7 \mathrm{~nm}, 64 \pm 5 \mathrm{~nm}$ and $60 \pm 5 \mathrm{~nm}$ and the CNE effective surface area to be $0.30 \pm 0.02 \mu \mathrm{m}^{2}, 1.33 \pm 0.09 \mu \mathrm{m}^{2}$ and $0.30 \pm 0.02 \mu \mathrm{m}^{2}$ for $\mathrm{P} 1, \mathrm{P} 2$ and $\mathrm{P} 3$ respectively. Among the three, the order for nanopore size is $\mathrm{P} 1>\mathrm{P} 2 \approx \mathrm{P} 3$ and the order for CNE size or surface area is $\mathrm{P} 1 \approx \mathrm{P} 3<<\mathrm{P} 2$. The nanopore size of $\mathrm{P} 1$ and the $\mathrm{CNE}$ area of $\mathrm{P} 2$ are at the high ends of the distributions. These large geometry variations gave us a chance to understand the potential sensing mechanism better and demonstrate the robustness of the potential sensing mechanism. 

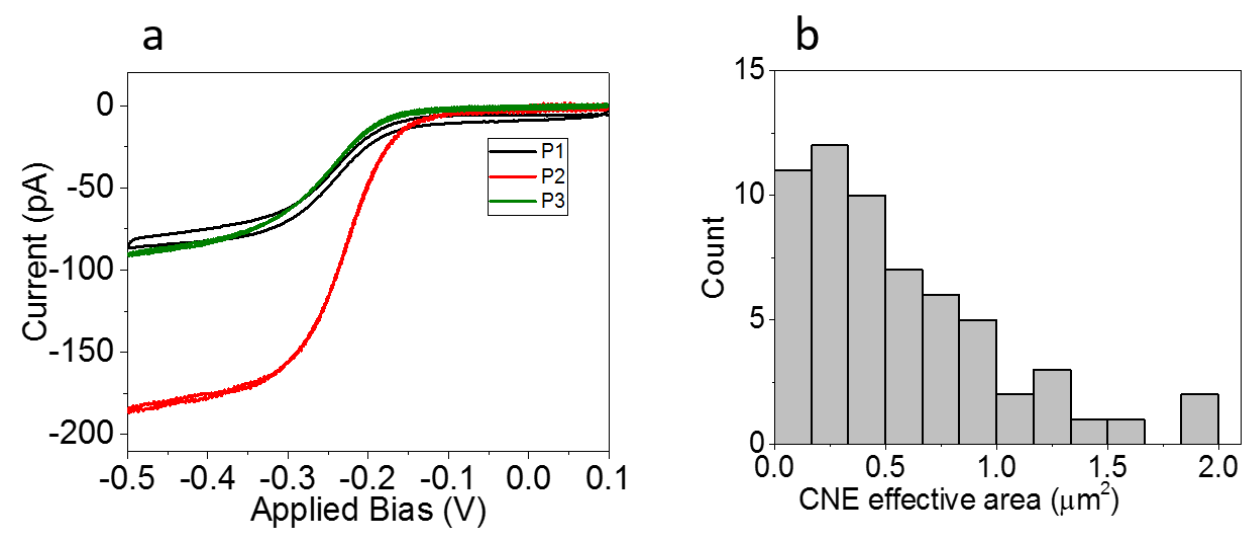

Figure 5.3. (a) CVs for multifunctional nanopipettes $\mathrm{P} 1, \mathrm{P} 2$ and $\mathrm{P} 3$ used in the experiment. CVs were recorded in $1 x$ PBS solution containing $1 \mathrm{mM} \mathrm{Ru}\left(\mathrm{NH}_{3}\right)^{6+}$ ions at a sweep rate $20 \mathrm{mV} / \mathrm{s}$. The black, red and green curves represent $\mathrm{CV}$ s for $\mathrm{P} 1, \mathrm{P} 2$ and $\mathrm{P} 3$ respectively. (b) The distribution of CNE effective area.

\subsection{2: Simultaneous Ionic Current and Potential Measurements of GNP Motion}

We used citrate-stabilized $40 \mathrm{~nm}$ GNPs as model NPs to demonstrate the capability of these multifunctional nanopipettes for simultaneous ionic current and potential measurements. The measurement setup and the details are given in chapter 2 under section 2.3.4 (see Figure 2.8). The bath solution is grounded and a positive bias is applied inside the nanopore. Therefore, the electric field drives the negatively charged GNPs towards the nanopore. The solution in the bath and nanopipette is $10 \mathrm{mM}$ PBS with $\mathrm{pH} 7.4$, unless specified otherwise. The GNPs are stable at this low salt concentration. The zeta potential of these GNPs is determined to be $-34.2 \mathrm{mV}$ in $10 \mathrm{mM}$ PBS by dynamic light scattering (DLS) method (see Chapter 2 in section 2.3.5). According to the colloidal theory, the measured zeta potential is at the slipping plane of the NP. At low salt concentration, we can ignore the stern layer and use Gouy-Chapman model $\left(\mathrm{V}=\mathrm{V}_{0} \mathrm{e}^{-\mathrm{x} / \delta}\right)$ to estimate the potential $\mathrm{V}_{0}$ at the NP surface. Here, $\delta$ is the Debye length and is about $2.8 \mathrm{~nm}$ in $10 \mathrm{mM}$ PBS. V is the zeta potential when $\mathrm{x}$ equals the slipping plane thickness $\mathrm{x}$ SP. We estimated 
XsP to be $1.9 \mathrm{~nm}$ (see section 2.3.5 in chapter 2). Therefore, the surface potential $\mathrm{V}_{0}$ of the GNP is about $66 \mathrm{mV}$ and the corresponding surface charge density is about $-24 \mathrm{mC} / \mathrm{m}^{2}$. Before adding GNPs in the solution, the current time traces (black color) are stable and featureless, for all the tested nanopipettes at various applied biases (an example is shown in trace (i) of Figure 5.4a). Meanwhile, there are only small and slow fluctuations in the potential time trace (red color). There is a nonzero potential difference in the potential time trace, which is about $-0.14 \mathrm{~V}$ for trace (i) in Figure 5.4a. For other measured nanopipettes, the nonzero potential baseline values varied from $-0.1 \mathrm{~V}$ to $-0.8 \mathrm{~V}$ though most values are at the lower magnitude end. The possible reasons for this large potential baseline are 1) different electrochemical potentials between a carbon electrode and $\mathrm{Ag} / \mathrm{AgCl}$ electrode and 2) the polarization of CNE. We also analyzed the noise spectra of both current and potential traces (see section 5.2.4 of this chapter). Between the two, the noise of potential data is much smaller and especially at high-frequency region.

With $40 \mathrm{~nm}$ GNPs in the bath solution, characteristic ionic current spikes and downward potential changes appear in the current and potential time traces after a certain waiting time. Figures 5.4-5.5 show several typical time traces of ionic current and potential, which were recorded simultaneously. The current spikes and potential changes always appear with strong one-to-one correspondence. These features are owning to the translocation of GNPs through the nanopore, confirmed by the appearance of GNPs inside the nanopipette barrel in the optical microscope images after the electrical measurements (see Figure 5.10). More GNPs appeared inside the nanopipette barrel when more current spikes and potential spikes/steps were observed in the measurements. 

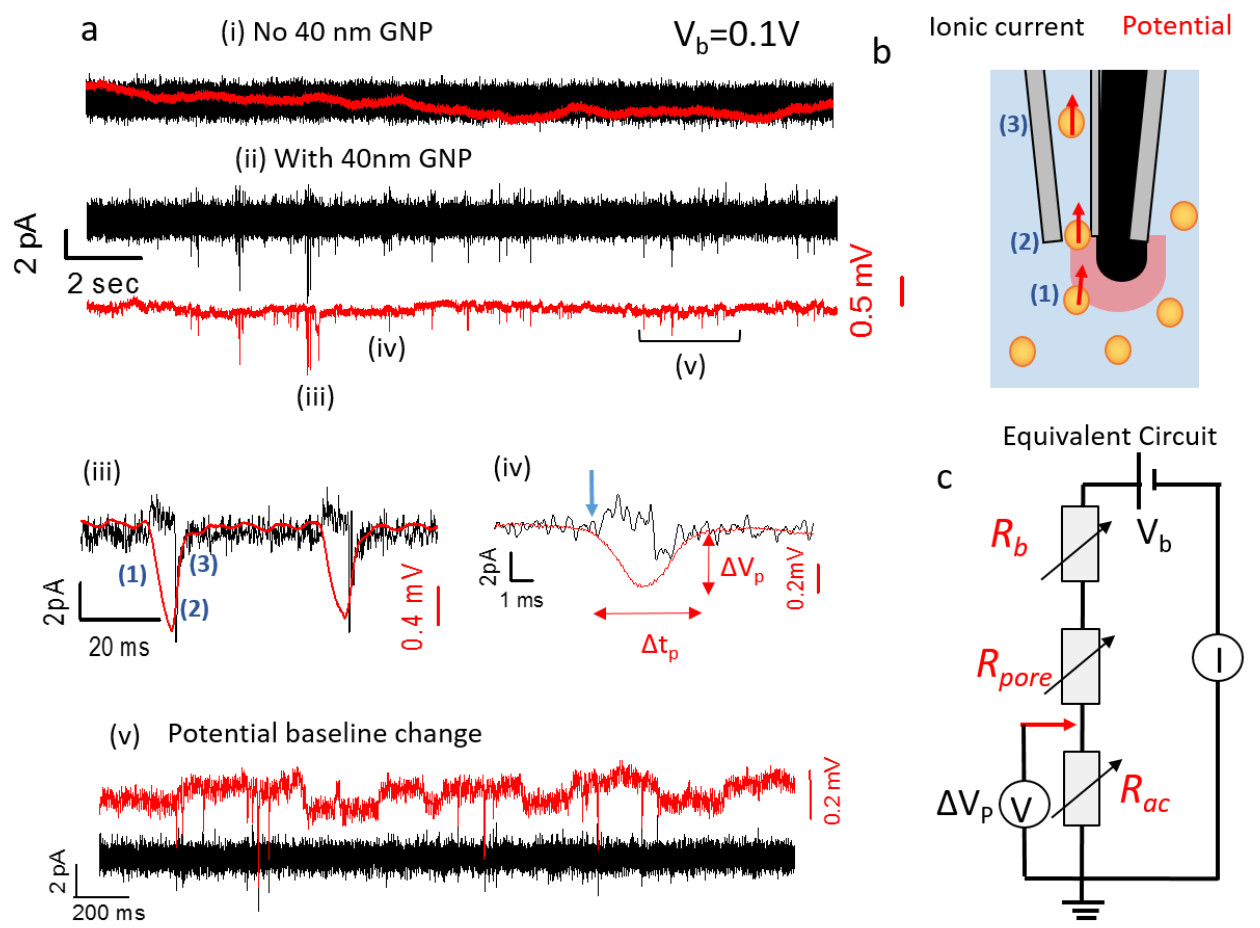

Figure 5.4. (a) The current (black) and potential (red) traces (20 s) at $\mathrm{V}_{\mathrm{b}}=0.1 \mathrm{~V}$ before (i) and after (ii) adding 40nm GNPs in the bath solution. The potential dips at (iii), (iv), and (v) positions of time trace (ii) are also displayed in zoom-in traces. All the data are from nanopipette P1. Both bath and nanopore filling solution are $10 \mathrm{mM}$ PBS. Both the current and potential traces are collected at $50 \mathrm{kHz}$ sampling rate and smoothed using the moving average method with $0.2 \mathrm{~ms}$ (10 points) window size for current and $2 \mathrm{~ms}$ (100 points) window size for potential. (b) A scheme to show the translocation of single GNP in three steps. The red color region represents the potential sensing zone. (c) The proposed equivalent circuit of the nanopore/CNE system.

Several groups have investigated the ionic current changes arising from the translocation of GNPs through a conical nanopipette. ${ }^{25-27}$ We noticed that our data are quite complicated. We attribute the complexity to two reasons: the measurements were carried out at low salt concentration and the GNPs accumulated near the nanopore entrance before 
translocation. It has been well-studied that the ionic current spike appears differently at low ionic concentration. ${ }^{8,28,29}$ At low ionic concentration, the surface charges of NP and nanopore play important roles and affect the distribution of ions within the nanopore. As a result, the current spike characteristics vary in shape and direction under different conditions. Entity accumulation behavior was observed when charged molecules were driven by electric force to enter a very small and charged CNT nanopore or silicon nanopore ${ }^{30,31}$ however, the accumulation/enrichment of a few tens nanometer-sized NPs at the nanopore entrance, and its effect on the ionic current signal, are less investigated. In our experiments, the GNP experienced a high entrance resistance for most of the tested nanopores, leading to frequent accumulation of GNPs near the pore orifice. This is based on several experimental observations: 1) A waiting time up to one hour (with an applied bias) is always needed before the first characteristic current, and potential changes occur. The waiting time is shorter with a higher applied bias or higher GNP bath concentration. We often applied a higher bias (i.e., $0.6 \mathrm{~V}$ ) at the beginning of the experiment and then used a smaller bias (i.e., $0.1 \mathrm{~V}$ ) to record data when events appear. After the appearance of translocation events, we noticed the event frequency gradually reduced. 2) The current spikes often appear in a burst of several spikes instead of individual spikes (see Figure 5.5). The number of spikes in each burst revealed the number of GNPs in the assembly near the nanopore. 3) In addition to the cluster of current spikes, we observed a dramatic change in potential baseline before the translocation, and staircase potential increase with a one-toone correspondence between the potential steps and the current spikes (see Figure 5.5). As we will discuss later, the accumulation and translocation of GNPs produced these potential changes. 4) Increased current spike frequency and reduced number of current spikes in 
each cluster are observed at higher applied biases. A higher electric force should help to overcome the entrance resistance and suppress the GNP accumulation, leading to more frequent translocation events and reduced GNP cluster size. Interestingly, excess noise appears in the potential noise spectra with the degree of GNP accumulation near the CNE (see section 5.2.3). We can also use the excess noise as a signature of GNP accumulation. The frequent GNP accumulation near nanopipette tip provides a good opportunity to investigate the nanoscale entity accumulation/enrichment and is a good test for the performance of these multifunctional nanopipettes.

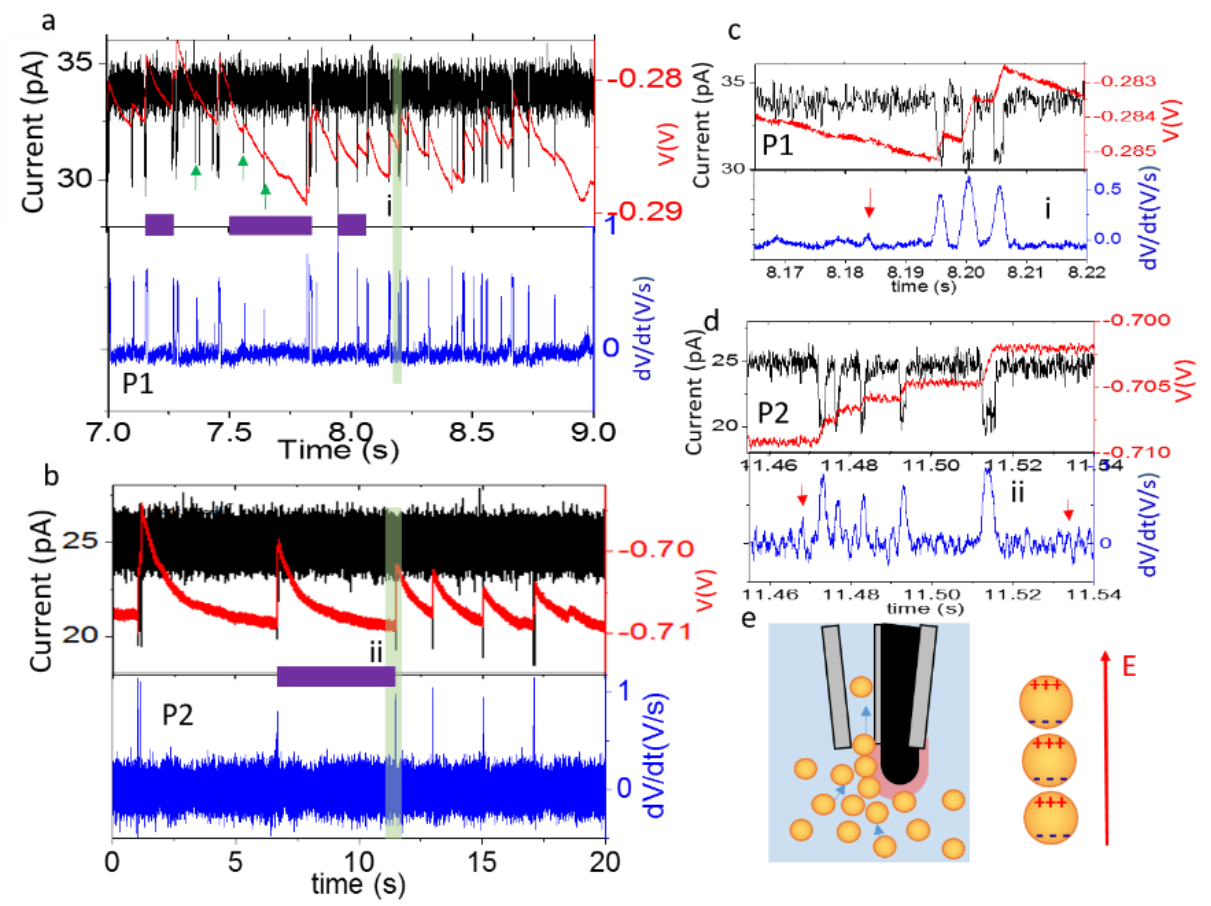

Figure $5.5(\mathrm{a}-\mathrm{b})$ The 20 seconds current (black), potential $(\mathrm{V}$, red) and first derivative of potential traces (dV/dt, blue) at $0.1 \mathrm{~V}$ (top) for P1(a) and P2 (b) after adding $40 \mathrm{~nm}$ GNPs in the solution. The purple strips indicated the full time span of potential dips. The green arrows indicate three current spikes appeared during the potential decrease stage of a potential dip. (c-d) The zoom-in traces of the green shaded regions of (a) and (b). The red arrows mark the small potential changes. $0.2 \mathrm{~ms}$ (10 points) moving average is applied to both current and potential data. (e) A scheme to show the accumulation of GNPs near the nanopipette tip and the possible wire cluster formation of GNPs before translocation. 
We have evaluated the entrance resistance for all the nanopipettes based on the waiting time, event frequency, cluster size, and cluster formation time before translocation. In a low ionic strength solution, the electrostatic repulsion between negatively charged NP and nanopore inner surface becomes significant, leading to a high entrance resistance for the GNP. To reduce the entrance resistance, strategies such as chemical modification are used to reduce the negative surface charge density of the glass surface. ${ }^{25}$ A pressure gradient is also applied to provide an extra driving force to help the negatively charged NPs overcome the nanopore entrance resistance. ${ }^{29}$ In a control experiment, we filled P3 nanopore with $25 \mathrm{mM}$ PBS solution to reduce the surface charge effect. The nanopores of P2 and P3 have the similar pore size. Compared with P2 nanopore filled with $10 \mathrm{mM}$ PBS solution, the translocation of GNPs through P3 nanopore became much easier. In addition to the electrostatic repulsion, the observed high entrance resistance for GNPs could also originate from the small nanopore size and the hindrance by the oversized CNE near the nanopore orifice. The CNE of $\mathrm{P} 2$ was overgrown and became much bigger than that of $\mathrm{P} 1$ and P3. Out of the three, the GNPs experienced the highest entrance resistance for P2 nanopore, showing few events and more GNPs in an accumulated cluster (see Figure 5.5 b\&d).

We also performed the same experiments using a dual-nanopore nanopipette with no $\mathrm{CNE}$ at the nanopipette tip (see Figure 5.8). As we will discuss later, the GNPs still accumulated near the nanopore entrance because of small nanopore size and electrostatic repulsion. However, the GNP translocation is much faster.

Before discussing potential changes, we will briefly discuss the current spikes. As we mentioned earlier, multiple mechanisms play important roles for the GNP 
translocations at low salt concentrations. Therefore, the current spikes can be either upward or downward at $10 \mathrm{mM}$ salt concentration, depending on the applied bias, the size and the surface charge of nanopore, and that of GNP. ${ }^{27,29,32}$ In addition, in all tested nanopipettes and at various measurement conditions, we observed bipolar, triangular and rectangular shaped current spikes. The triangular shape of current spike is attributed to the typical electrophoretic transport of GNPs through conical shape nanopore. ${ }^{25}$ The rectangular shape current spike is attributed to the slower translocation of GNPs. The GNPs may be slowed down by the opposite electroosmotic flow,${ }^{8}$ the interactions with nanopore surface, or the high entrance resistance of the nanopore. The bipolar spikes are likely due to the surface charge effects of charged GNPs and the nanopore inner surface. ${ }^{29}$ Thorough investigations are needed for these ionic current changes, and they are beyond the scope of this paper. It is worth to point out that whichever the shape and direction of current spikes, corresponding potential changes always appear simultaneously.

We will now focus on the observed potential changes. Although GNP accumulation happens more often in these multifunctional nanopipettes, we will first discuss the potential changes induced by single GNP translocation events with weak or no GNP accumulation. The trace (ii) of Figure 5.4a shows a typical current and potential trace (20 seconds long) of this type, recorded from P1 at $0.1 \mathrm{~V}$. Downward current spikes and potential dips are observed. The zoom-in traces of big (trace (iii)) and small (trace (iv)) potential dips (red color) and the corresponding current spikes (black color) are also displayed. The current spike is bipolar, showing both upward and downward changes in one event. For the potential dip, the potential began to drop at almost the same time when the current began to change (indicated by the blue arrow in trace (iv)). The duration of a bipolar current spike 
is almost the same as the duration $\Delta \mathrm{t}_{\mathrm{p}}$ of a potential dip. The same duration time suggests a single GNP translocation event generates a potential dip. We also observed rectangular shape potential baseline changes, as shown in the zoom-in trace (v). The magnitude of these potential baseline changes is very small (about $0.1 \mathrm{mV}$ ) and the changes can last for a few seconds. We did not find corresponding current changes for these rectangular potential baseline changes. These potential changes are likely due to the small motion of GNPs near the CNE potential sensing zone and outside the nanopore orifice. Because the extrusion of the CNE, the non-translocating motion of GNP does not affect the ionic current but can affect the CNE potential if the GNPs are very close to the CNE surface.

What is the potential detection mechanism for the multifunctional nanopipette? We have carried out FEM simulations to understand the potential detection mechanism in this system. We will first explain qualitatively, and the quantitative simulation results are shown in Figure 5.13 \&5.14 in simulation sections. Obviously, the potential of a GNP can be detected based on the direct potential or charge sensing mechanism: the presence of a charged GNP alters the potential of a nearby potential probe. However, the sensing range of this mechanism is very short in ionic solution because of the charge screening effect. Based on Gouy-Chapman model $\left(\mathrm{V}=\mathrm{V}_{0 \mathrm{e}^{-\mathrm{x} / \delta}}\right)$, only a $20 \mu \mathrm{V}$ potential change can be detected when a GNP with a surface potential $66 \mathrm{mV}$ is about $23 \mathrm{~nm}$ away from the potential probe. For a nanopore device, a voltage divider sensing mechanism is also available, as reported by Xie et.al.. ${ }^{2}$ To understand how this sensing mechanism works in our multifunctional nanopipette, we draw an equivalent circuit as shown in Figure 5.4c.Three variable resistors are connected in series. $R_{a c}, R_{\text {pore }}$ and $R_{b}$ are the access resistance, nanopore resistance, and barrel resistance respectively. The potential of the bath 
solution is $0 \mathrm{~V}$ (grounded), and the potential deep inside the barrel of the nanopore side is $\mathrm{V}_{\mathrm{a}}$, the applied bias. The CNE is treated as a point probe and positioned at the location

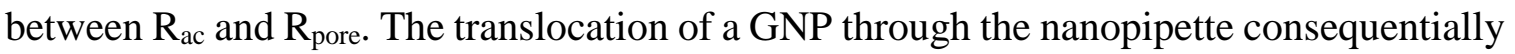
increases the magnitudes of $R_{a c}, R_{\text {pore }}$ and $R_{b}$. These resistance changes alter the potential distribution in the circuit, and therefore the potential at the potential probe CNE. For this sensing mechanism, the GNP has a long-range impact on the potential at the CNE even the GNP is inside the nanopipette barrel and several hundred nanometers away from the orifice, owning to the small conical angle of the nanopipette. Our simulation reveals that both sensing mechanisms contribute to the observed results. Figure $5.4 \mathrm{~b}$ illustrates the typical motion of a GNP during the translocation event in three steps: (1) approaching the nanopipette tip and entering the CNE potential sensing zone (red color area); (2) translocating through the nanopore orifice; (3) moving inside the narrow barrel of the nanopore side. We also labeled these steps in a potential dip shown in Figure5.4a (iii). At step 1, the voltage divider model predicts a potential increase (less negative) at the CNE because the presence of GNPs near the nanopore entrance increases the magnitude of $R_{a c}$ and therefore increases the potential drop at the $\mathrm{R}_{\mathrm{ac}}$. In the opposite, the direct potential sensing model predicts a potential decrease (more negative) at the CNE for the approaching of negatively charged GNPs. Therefore, the two mechanisms compete with each other. The always downward potential dips observed in the experiments suggested the direct potential/charge sensing mechanism dominates step 1 at our experimental conditions. At step 2, the screening of GNP surface charge by the quartz wall and the magnitude increase of $\mathrm{R}_{\text {pore }}$ both lead to a potential increase at the $\mathrm{CNE}$. Therefore, both mechanisms contribute positively to the potential increase at the CNE. This leads to a sharp increase in the 
potential dip. At step 3, because the GNP is inside the barrel and far away from the CNE, the direct potential/charging sensing mechanism fails. Only voltage divider model works at step 3 and the gradual decrease of $\mathrm{R}_{\mathrm{b}}$ leads to a gradual increase of potential at CNE (as well as current). The potential will return to the original potential baseline when the GNP is deep inside the barrel.

We performed the statistical analysis of a large number of isolated potential dips. From the scatter plot and histograms in Figure 5.6, a single GNP translocation event induced a mean duration time $\Delta \mathrm{t}_{\mathrm{p}}$ about $4.2 \pm 0.8 \mathrm{~ms}$ and a mean potential change $\Delta \mathrm{V}_{\mathrm{p}}$ about $0.27 \pm 0.14 \mathrm{mV}$ at the CNE. The maximum potential change is less than $2 \mathrm{mV}$. Obviously, the potential change is only a small fraction of the GNP zeta potential. Because the measured potential change magnitude is mainly caused by the surface potential/change of the GNP, the small potential change suggests the GNPs keep a small distance away from the CNE during the translocation.

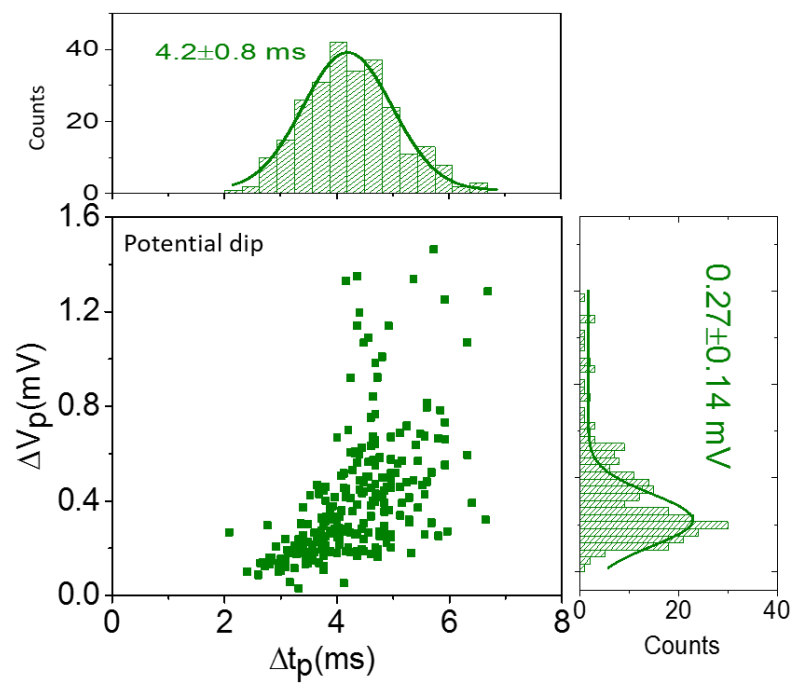

Figure 5.6. The scatter plot and histograms of potential dip height $\Delta \mathrm{V}_{\mathrm{p}}$ and width $\Delta \mathrm{t}_{\mathrm{p}}$ from 259 potential dips similar to (iii) and (iv) of 4.4 (a). The solid lines of the histograms are Gaussian fits. 
As we mentioned earlier, the GNPs often accumulate near the nanopore orifice because a nanopore with a high entrance resistance can hinder the GNP translocation. The presence of multiple GNPs in the CNE sensing zone makes cumulative changes to the potential change at the $\mathrm{CNE}$, which we can use to study the dynamic accumulation of GNPs. Our results clearly demonstrate this. Figure 5.5a shows another type of time trace from P1. The current (black curve) baseline is still very stable, but the potential baseline (red curve) varied significantly, showing a large number of saw-tooth type big potential dips. More regular and bigger potential dips are observed for P2 (Figure 5.5b). The shape of these potential dips in Figures 5.5a and 5.5b (see three examples marked by purple color strips) is very different from the potential dips induced by individual GNP translocation events in Figure 5.4. In each large potential dip, the potential first drops continuously, then gradually flats out (this stage is missing in Figure 5.5a from P1 but is obvious in Figure 5.5b from P2), finally rises up sharply. Again, there is no corresponding change in the ionic current for the majority part of a potential dip. Corresponding current changes are only found in the fast rising edge, as shown in Figure 5.5c and 5.5d, two zoom-in traces with expanded fast rising edges. Clearly, the fast rising edge of each potential dip contains multiple potential steps and aligns with the same number of current spikes. The current spikes are of rectangular shape with the same height. These current spikes confirm that the staircase type of fast-rising potential is the result of continuous translocations of multiple GNPs. The accumulation of several GNPs towards the nanopipette tip is likely the reason for the large potential dip before the translocations. It is interesting to note that these rectangular current spikes are also different from the bipolar ones from single GNP translocation events (see Figure 5.4). For all the pipettes, we mainly observe rectangular 
shaped current spikes and occasionally triangular shaped current spikes when the GNP translocation happens after the GNP accumulation step. The accumulation of charged GNPs may affect local ion and potential distributions as well as the GNP translocation motion.

The difference in the nanopore entrance resistance of P1 and P2 contributes to the difference in the potential data. For P2 with a bigger entrance resistance, the frequency of potential dips are much less, but the magnitudes are bigger, suggesting more GNPs accumulated at the nanopore orifice before the coupled translocation. Meanwhile, the accumulated GNPs near the nanopipette tip should leave enough rooms to allow ions to pass, suggested by the unchanging ionic current baseline. As shown in P1 data (Figure 5.5a), individual potential steps (indicated by green arrows) with their corresponding current spikes are often observed during the continuous potential drop stage. These single GNP translocation events interrupted the GNP accumulation, preventing a large GNP cluster to form outside the P1 nanopore orifice. Compared with the potential dips of P2, the time duration for a potential dip of P1 is much shorter. The potential dip of P2 often contains a flat bottom, suggesting the GNP assembly reaches a dynamic equilibrium. No flat region is observed for the short-lived potential dips in P1 data. Furthermore, the fast rising edge of a potential dip typically contains no more than three steps for P1. In contrast, there are more than five steps are observed in the fast rising edge of a potential dip from P2, suggesting the GNP cluster formed outside the P2 nanopore entrance contains more than five GNPs (see Figure 5.5d).

The potential traces in Figure 5.5 are complicated because there are cumulative contributions from several GNPs. The large potential baseline changes due to GNP 
accumulation overshadowed the smaller potential changes caused by single GNP translocation. Therefore, we plotted the first derivative $(\mathrm{dV} / \mathrm{dt})$ of all the potential traces (blue curves) in Figure 5.5. In the dV/dt plot, the slow baseline change becomes very small. In contrast, the fast potential change caused by single GNP translocation at the nanopore mouth becomes prominent. One-to-one correspondence between current spikes and the first derivative potential peaks is immediately clear. The magnitude and sign of $\mathrm{dV} / \mathrm{dt}$ reflect the speed and direction of moving GNPs. The small negative baseline (about several $\mathrm{mV} / \mathrm{s}$ in magnitude) in these derivative plots suggests that the assembled GNPs approach very slowly toward the nanopipette tip and are almost motionless (for P2) before several coupled single GNP translocation events begin. The positive peaks with more than two orders higher magnitude around $0.5-1 \mathrm{~V} / \mathrm{s}$ suggest the translocating speed of GNP at the nanopore orifice is much faster than the approaching speed of GNP outside the nanopore orifice. The $\mathrm{dV} / \mathrm{dt}$ trace also reveals small potential changes, as indicated by the red arrows in Figures 5.5c and 5.5d. Such small variations are found to appear more often in the baseline of $\mathrm{P} 2 \mathrm{dV} / \mathrm{dt}$ trace (Figure 5.5d). On closer examination, we can barely recognize corresponding current changes at several positions in the current trace. These small changes are likely due to the non-translocation motions of GNPs near the CNE sensing zone. It is expected that more GNPs accumulated near the P2 tip region will generate more nontranslocation events. Without analyzing the current and potential changes together, we cannot notice these non-translocation events. Interestingly, we did not recognize any hitn-stay collision events by GNPs at the CNE. These events should cause much larger stepwise potential changes at the $\mathrm{CNE}$ with no corresponding ionic current changes at the nanopore. Optical microscope images also reveal no signs of large GNP aggregations at 
the tip of the nanopipette. In contrast, we always observed GNP aggregations inside the nanopipette barrel after translocation experiments. A plausible reason is that the GNPs cannot stick to the CNE surface with a high curvature. The hit-n-run collision events by GNPs may happen at the CNE, and these events may be too fast to be observed.

The dramatic drop in potential baseline and the staircase type potential increase provide information of the dynamic GNP accumulation-translocation process. Figure 5.5e illustrates a proposed mechanism. With an applied bias, GNPs move towards the nanopore entrance by electrophoresis. Near the nanopore entrance, the nanopore stops the approaching GNPs and induces GNP accumulation. When the entrance resistance of the nanopore is big enough, the nanopore can keep a large number of GNPs outside the orifice, and the GNP assembly can reach a dynamic equilibrium, revealed by the almost zero $\mathrm{dV} / \mathrm{dt}$ for $\mathrm{P} 2$ at $0.1 \mathrm{~V}$. The $\mathrm{dV} / \mathrm{dt}$ curve also revealed the frequent non-translocation motions of these GNPs while waiting outside the nanopore entrance. We speculate the GNPs may cluster in a pearl chain formation outside the nanopore orifice, similar to previous observations of GNP pearl-chain assembly in a non-uniform AC electric field. ${ }^{33,34}$ In the bulk solution, the charged GNPs are stabilized by electrostatic repulsion. Near the nanopore entrance, they become highly concentrated. Pushed by other GNPs from behind, the GNPs near the pore mouth can overcome the electrostatic repulsion and become very close to each other. Because the electric field is greatly enhanced near the nanopore mouth and close to the GNP surface $\left(\sim 1 \times 10^{7} \mathrm{~V} / \mathrm{m}\right.$, see Figure $\left.5.13 \mathrm{a}\right)$, these polarized GNPs become very close to each other or even make contact in a chain formation because of the attractive mutual dielectrophoretic (DEP) force. ${ }^{35}$ The protruded CNE geometry and the relatively large effective electrode area allow more GNPs to reach the sensing zone of the 
CNE and affect the CNE potential cumulatively. The increased number of GNPs inside the potential sensing zone and the decreased distance between GNP and CNE lead to a continuous and gradual potential drop at the CNE. At this step, the direct potential/charge sensing mechanism still dominates, supported by the unchanging ionic current baseline. The accumulation helps the GNPs to overcome the nanopore entrance resistance. With the accumulation of negatively charged GNPs near the nanopore, the electrostatic repulsion between GNPs, the GNP concentration gradient, and the electrophoresis are all increased, and they provide additional driving forces to push the GNPs to enter the nanopore. Eventually, the first GNP in the cluster enters the nanopore, leaving the others in the cluster outside the nanopore. The stronger attractive force between GNPs in the cluster may drive all the GNPs in the cluster to enter the nanopore one after the other. As a result, the staircase type potential increase appears. The number of steps indicates the number of GNPs in the chain cluster. The chain can be formed much longer outside P2 nanopore. While the GNPs in the chain cluster enter the nanopore, the GNPs behind also move forward to fill the vacancy and form a new chain cluster. Correspondingly, a continuous potential drop shows up again, as suggested by the experimental results in Figure 5.5. The cluster formationtranslocation cycle can repeat many times at the nanopore entrance, which creates a sawtooth potential change pattern. Because of the continuous GNP movements outside the nanopore, the gradual potential increase due to the single GNP movement inside the nanopore (step 3 in Figure 5.4b) cannot be detected.

We analyzed the characteristics of a large number of potential steps as well as their corresponding current spikes. For the same nanopipette, although the event frequency increases with the increased applied bias, no obvious bias dependence is observed for the 
duration and magnitude of potential steps (as well as the two parameters of current spikes), which is likely diminished by the accumulation behavior of GNPs. We, therefore, combined the data at different applied biases. Figure 5.7a shows the intensity plot of the potential step height $\Delta \mathrm{V}_{\mathrm{s}}$ versus step width $\Delta \mathrm{t}_{\mathrm{s}}$, which is composed of 1847 potential steps from $\mathrm{P} 1$ at $0.1 \mathrm{~V}$. The maximum $\Delta \mathrm{V}_{\mathrm{s}}$ is about $6 \mathrm{mV}$, which is much smaller than the zeta potential of a GNP. Therefore, these events are unlikely from hit-n-stay or hit-n-run collision events from GNPs when accumulating outside the nanopore. For comparison, Figure 5.7b shows the intensity plot of corresponding current spike results. The potential step height $\Delta \mathrm{V}_{\mathrm{s}}$ is $0.32 \pm 0.23 \mathrm{mV}$ and the step width $\Delta \mathrm{t}_{\mathrm{s}}$ is $1.0 \pm 0.3 \mathrm{~ms}$. The $\Delta \mathrm{V}_{\mathrm{s}}$ is slightly bigger than $\Delta \mathrm{V}_{\mathrm{p}}$ showing in Figure 5.7 while $\Delta \mathrm{t}_{\mathrm{S}}$ is much shorter than $\Delta \mathrm{t}_{\mathrm{p}}$. The obvious difference between $\Delta t_{s}$ and $\Delta t_{p}$ supports our assumption in the previous paragraph that the translocating GNP is pushed by additional driving forces and enters the nanopore faster when there are GNP accumulations at the nanopore entrance. The current spike height $\Delta \mathrm{I}_{\mathrm{p}}$ is $3.1 \pm 0.9 \mathrm{pA}$ and the current spike width $\Delta \mathrm{t}_{\mathrm{I}}$ is $1.0 \pm 0.3 \mathrm{~ms}$. There is no noticeable difference in the mean values and the distributions between $\Delta \mathrm{t}_{\mathrm{s}}$ and $\Delta \mathrm{t}_{\mathrm{t}}$. This confirmed once again that the GNP translocation event caused the potential step. From Figure 5.7a we also find $\Delta \mathrm{V}_{\mathrm{s}}$ is proportional to $\Delta \mathrm{t}_{\mathrm{s}}$, as revealed by the green dash guideline. In contrast, $\Delta \mathrm{I}_{\mathrm{I}}$ is independent of $\Delta \mathrm{t}_{\mathrm{I}}$ (Figure 5.7b). This is because ionic current change $\Delta \mathrm{I}_{\mathrm{I}}$ is more sensitive to the size of GNP and $\Delta \mathrm{V}_{\mathrm{s}}$ is more sensitive to the distance between GNP and CNE. The slower GNP translocation events should have opportunities to move closer to the CNE and cause bigger potential changes at the CNE. As shown in Figure 5.7a-b, we divide all the events of P1 into (i) fast ( $\left.\Delta \mathrm{t}_{\mathrm{I}}<1 \mathrm{~ms}\right)$, (ii) medium ( $\left.1 \mathrm{~ms}<\Delta \mathrm{t}_{\mathrm{I}}<3 \mathrm{~ms}\right)$ and (iii) slow $\left(\Delta \mathrm{t}_{\mathrm{I}}>3 \mathrm{~ms}\right)$ regions. There are about $38 \%$ fast events $\left(\Delta \mathrm{t}_{\mathrm{I}}<1 \mathrm{~ms}\right)$ for $\mathrm{P} 1$ and about $16 \%$ 
for P2. Thus, the entrance resistance of nanopore certainly plays a role in slowing down the GNP translocation. We also showed two representative events at region (i) and (iii) in Figure 5.7c. The potential (red) and current (black) changes begin and end almost at the same time. It is also obvious that the magnitude of $\Delta \mathrm{I}_{\mathrm{I}}$ is almost the same while the magnitude of $\Delta \mathrm{V}_{\mathrm{s}}$ is much bigger for the slow event (iii). In (iii), the potential baseline slope before and after the potential step is obvious and similar. Therefore, the single GNP translocation event is coupled with the collective and slow approaching movement of GNPs outside the nanopore. The slower event (iii) showed much better signal-to-noise ratio (SNR) than the fast event (i). Because the GNP translocation is slower when the nanopore entrance resistance is higher, we can improve the SNR of potential measurement by using a nanopore with a large entrance resistance. 

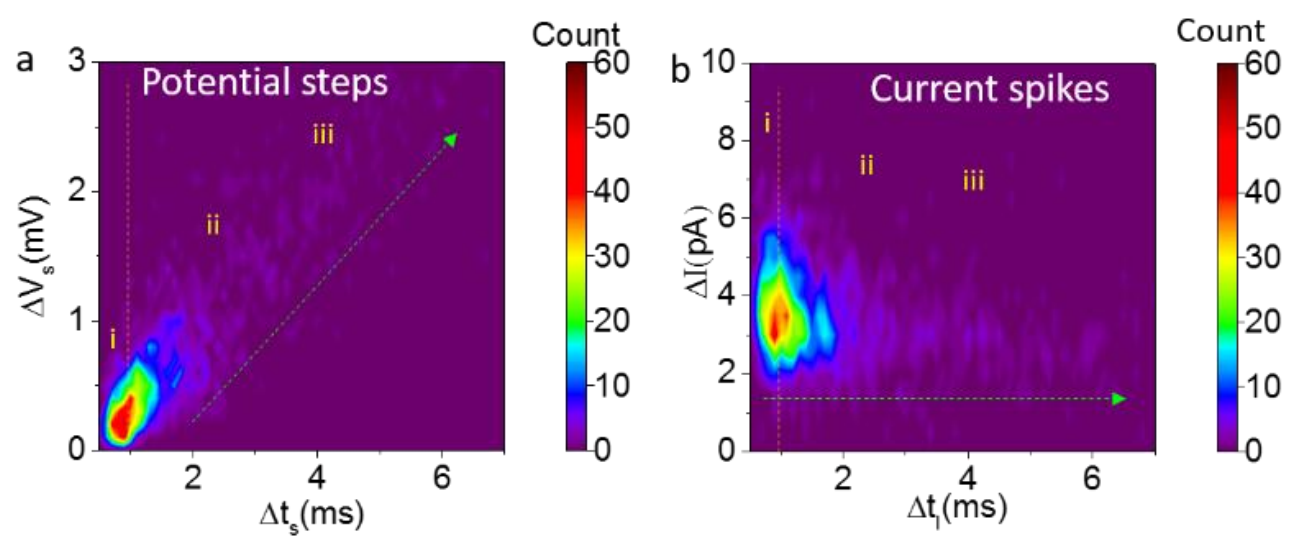

C (i)

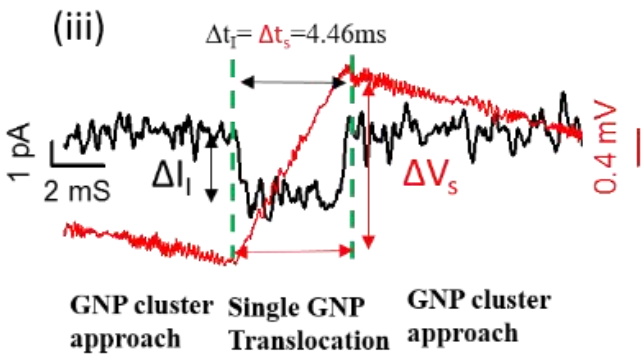

Figure 5.7. (a-b) The intensity plots of the distributions of height and width of the potential steps (a) and corresponding current spikes (b) of P1 nanopore and CNE data. The yellow (at $1 \mathrm{~ms}$ ) and green dash lines are guides to the eye. (c) Two representatives (i) fast and (iii) slow translocation events with the potential steps (red color) and corresponding current spikes (black). The green dash lines indicate the start and end points for potential steps and current spikes. $0.2 \mathrm{~ms}$ (10 points) moving average is applied to both current and potential data.

Dual-nanopore nanopipette without $\mathrm{CNE}$ was also used for simultaneous ionic current and potential measurements. ${ }^{36}$ The conductive electrolyte in one barrel replaced the conductive $\mathrm{CNE}$ as the potential sensing probe. We compared the difference in potential detection between dual-nanopore nanopipette and CNE/nanopore nanopipette. The first notable difference is that the chance to observe ionic current spikes resulted by GNP translocations is significantly lower when using dual-nanopore nanopipettes. However, the optical microscope images (see Figure 5.10) always indicated that the GNP translocation events indeed happened. One plausible explanation is that many events happen too fast to be detected. The data shown in Figure 5.8 supported this. The mean current spike width $\Delta \mathrm{t}_{\mathrm{I}}$ 
is about $0.6 \mathrm{~ms}$ for a dual-nanopore nanopipette with a very small nanopore size $44 \pm 4 \mathrm{~nm}$ at $0.5 \mathrm{~V}$. This $\Delta \mathrm{t}_{\mathrm{I}}$ is obviously shorter than the ones we observed from $\mathrm{P} 1, \mathrm{P} 2$ or $\mathrm{P} 3$. We seldom observed current spikes from a nanopore with size over $60 \mathrm{~nm}$. Therefore, the GNPs should translocate much faster through the nanopore of a dual-nanopore nanopipette. The presence of CNE slowed down the translocation of GNP and therefore made the translocation event easier to detect in both ionic current and potential signals. The current spikes also appeared in cluster style (see Figure 5.8c), suggesting that the large entrance resistance for negatively charged GNPs still exists for these dual-nanopore nanopipettes. The current spikes are often upward, resulting from the significant overlap of double layers. We observed saw-tooth type potential baseline changes as well. However, the fine structures such as staircase type potential steps are lost. Thus, the CNE is critical for realizing single GNP sensitivity. We also noticed that even the cumulative baseline potential changes were not easy to be observed when using these dual-nanopore nanopipettes. These control experiment results highlighted the advantage of using $\mathrm{CNE} /$ nanopore nanopipette for simultaneous current and potential measurements of GNP translocation events. 

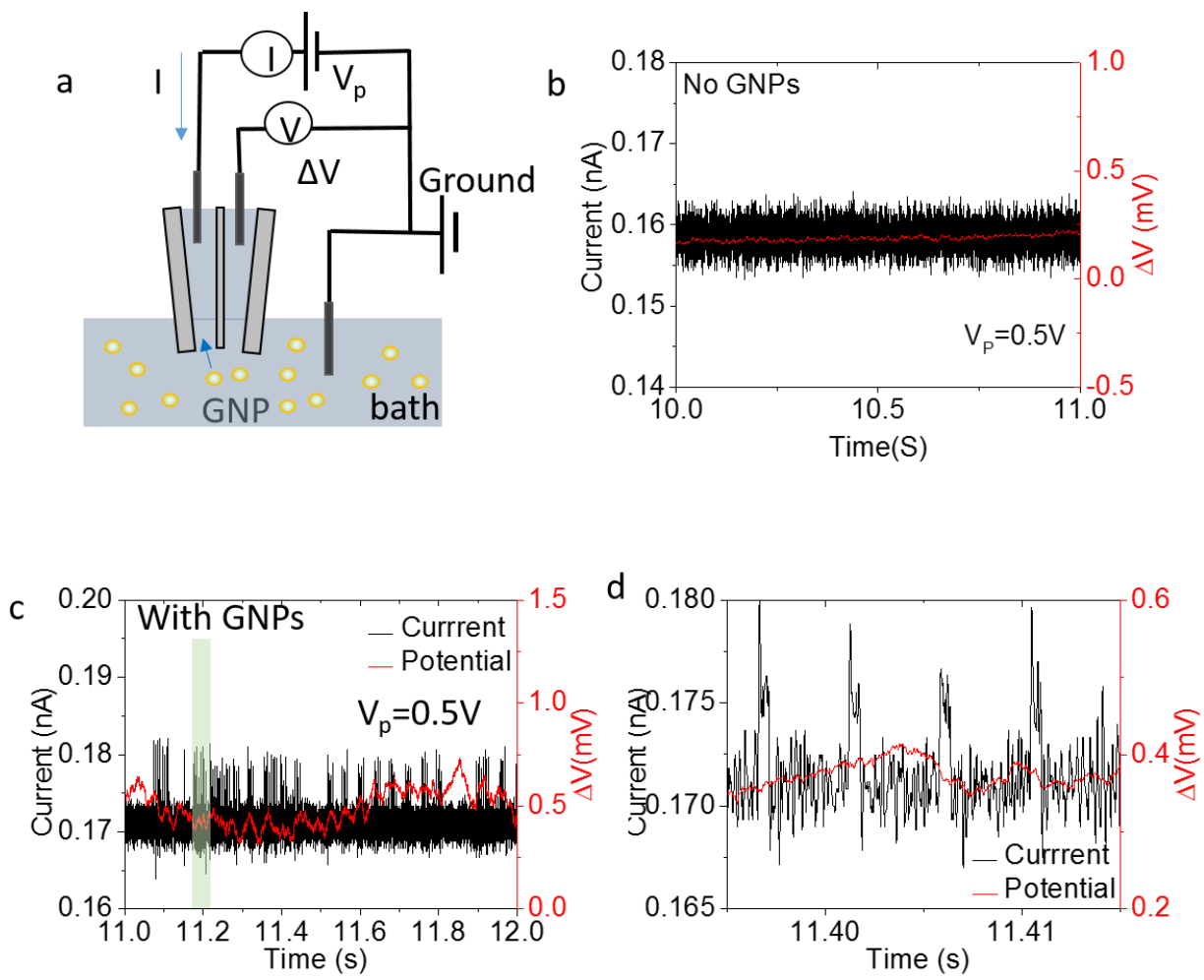

Figure 5.8. (a) The setup for the measurement using dual-nanopore nanopipette. (b) The current (black) and potential (red) time traces (1s) before adding GNPs ( $\mathrm{Vp}=0.5 \mathrm{~V})$. (c) The current (black) and potential (red) time traces (1s) after adding GNPs ( $\mathrm{Vp}=0.5 \mathrm{~V})$. (d) The zoom-in trace of the green color shaded region to show individual rectangular shape current spikes and the corresponding potential change.

The size of the nanopore is $44 \pm 4 \mathrm{~nm}$. For this size of the nanopore, upward and square shape current spikes were often observed at low salt concentration. Figure 5.8 (c-d) showed the typically upward current spikes at 10mM PBS. Current spikes showed uniform magnitude at $8 \pm 1 \mathrm{pA}$ and the width of these spikes is $0.62 \pm 0.02 \mathrm{~ms}$ ( see Figure 5.9). The current spike magnitude is bigger than $\mathrm{P} 1$ and $\mathrm{P} 2$ nanopipettes described above, which is due to the comparable size between GNPs and the nanopore here. The width of these spikes is shorter than the one of P1, suggesting GNPs translocate faster through the nanopore. As shown in Figure 5.8(c) and (d), we also observed familiar saw-tooth potential changes. 
These potential changes can roughly correlate to the spike clusters. However, the one-toone correspondence is lost.
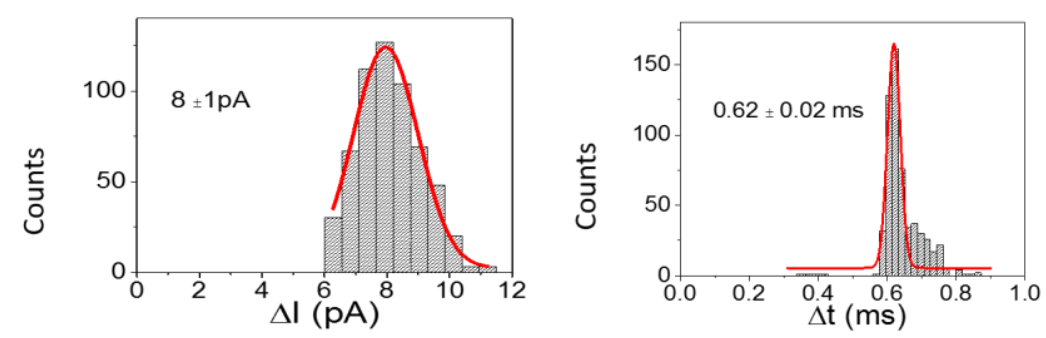

Figure 5.9. The histograms for spike height $\left(\Delta \mathrm{I}_{\mathrm{I}}\right)$ and spike width $\left(\Delta \mathrm{t}_{\mathrm{I}}\right)$ of 583 current spikes with dual nanopore nanopipette. The red curves are the Gaussian fits.

We also observed downward current spikes from other measurements. In summary, upward current spikes are more often observed (1) when the size of the nanopore is close to the GNP size, (2) at a higher applied bias and (3) at lower salt concentrations. For some nanopores, we observed downward spikes at $0.1 \mathrm{~V}$ but upward current spikes at higher bias, which is likely due to the stronger electroosmotic flow at a higher applied bias. With 25 mM PBS bath solution, the chance to observe downward current spikes are more often.

5.2.3: Optical Microscope Images of GNPs Inside the Nanopipettes After Translocation Experiments

(i) Dual nanopore nanopipette

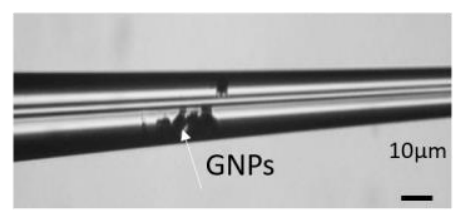

(ii) $\mathrm{CNE} /$ nanopore nanopipette

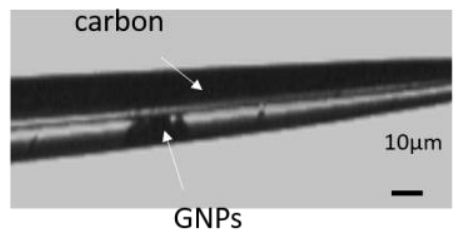

Figure 5.10. Optical microscope images for (i) dual-nanopore nanopipette and (ii) $\mathrm{CNE}$ /nanopore nanopipette after GNP translocation experiments. The GNP aggregates are clearly visible inside the nanopipette barrel. 


\subsection{4: Noise Analysis of Ionic Current and Potential Measurements}

The potential amplifier is battery powered, and the only $\mathrm{x} 10$ gain is used. Therefore the bandwidth for potential measurement can be much larger than the current measurement. In the measurements, we used $5 \mathrm{kHz}$ and $40 \mathrm{kHz}$ low-pass filter bandwidth for current and potential measurements respectively. We compared the noise in ionic current and potential data in our measurements. The noise power spectrum density (PSD) S(f) is obtained by performing Fast Fourier Transformations (FFT) on a current or potential time trace (one second) at 0.1V. Figure 5.11 (a) and (b) showed the normalized PSDs of current $\left(\mathrm{S}_{\mathrm{I}} / \mathrm{I}^{2}\right)$ and potential $\left(\mathrm{S}_{\mathrm{v}} / \mathrm{V}^{2}\right)$ for P1, P2 and P3 before adding GNPs. It is obvious that the noise of potential data is much smaller than that of ionic current data, especially at high frequency $(>100 \mathrm{~Hz})$. The potential noise spectra display characteristic $1 / \mathrm{f}$ noise or flicker noise at low frequency $(<100 \mathrm{~Hz})$. The potential noise spectral density distribution flats out at higher frequency with reduced magnitude. In contrast, the ionic current noise spectra show capacitance noise, which increases with the frequency. The noise analysis suggests the potential measurement is better for faster event measurement. We also compared the potential noise of P1, P2 and P3 before adding GNPs in the bath solution. Overall, the noise of $\mathrm{P} 1$ is the smallest, and the noise of $\mathrm{P} 2$ is the biggest. The CNE surface area may play a role here. There should be an optimized CNE geometry and surface area considering the balance among noise, sensitivity and sensing range. After adding GNPs, we noticed noise increase in the potential noise spectra. The noise is related to the accumulation of GNPs. As shown in Figure 5.11(c), no obvious change is observed in the noise spectra immediately after adding GNPs. With the accumulation of GNPs near the nanopipette tip, the change in noise spectra becomes obvious. Low-frequency noise $(<100 \mathrm{~Hz})$ is increased 
with the GNP accumulation, which is related to the observed potential baseline change. At high frequency, a broad bump appears between 1-10 k Hz. This high-frequency noise (also see Figure 5.11 (f)) is stronger after a longer accumulation time. This phenomenon is interesting, and the exact reason needs further investigation. Figure 5.11 (d) compares the noise spectra of P1, P2 and P3 after the heavy accumulation of GNPs, suggested by event frequency. The same high-frequency noise can be barely found in the noise spectra of P3 but cannot be resolved in the noise spectra of P2. This is because the noise level of P2 and $\mathrm{P} 3$ is higher.

Before data analysis, we normally applied 10 points $(0.2 \mathrm{~ms}$ window size $)$ to both the current and potential time traces to reduce noise. Figure 5.11 (e) shows one example before and after the 10 points moving average smoothing. The zoom-in trace in Figure 5.11 (f) illustrates that the 10 points moving average did not change the time duration and magnitude of a fast current spike and potential step ( $<1 \mathrm{~ms})$. The Figure 5.11(f) is a noisier trace from P1. The meter picked up spurious high frequency $(>1 \mathrm{k} \mathrm{Hz})$ and $60 \mathrm{~Hz}$ powerline noises. Even in this condition, the potential step is still resolvable in the raw data. After smoothing, the peak-to-peak potential noise is reduced from $0.4 \mathrm{mV}$ to $0.1 \mathrm{mV}$, and the potential step is obvious. We sometimes also used 100 points moving average $(2 \mathrm{~ms}$ window size) when the events are slower than $2 \mathrm{~ms}$. The peak-to-peak potential noise can be smaller than $10 \mu \mathrm{V}$. 

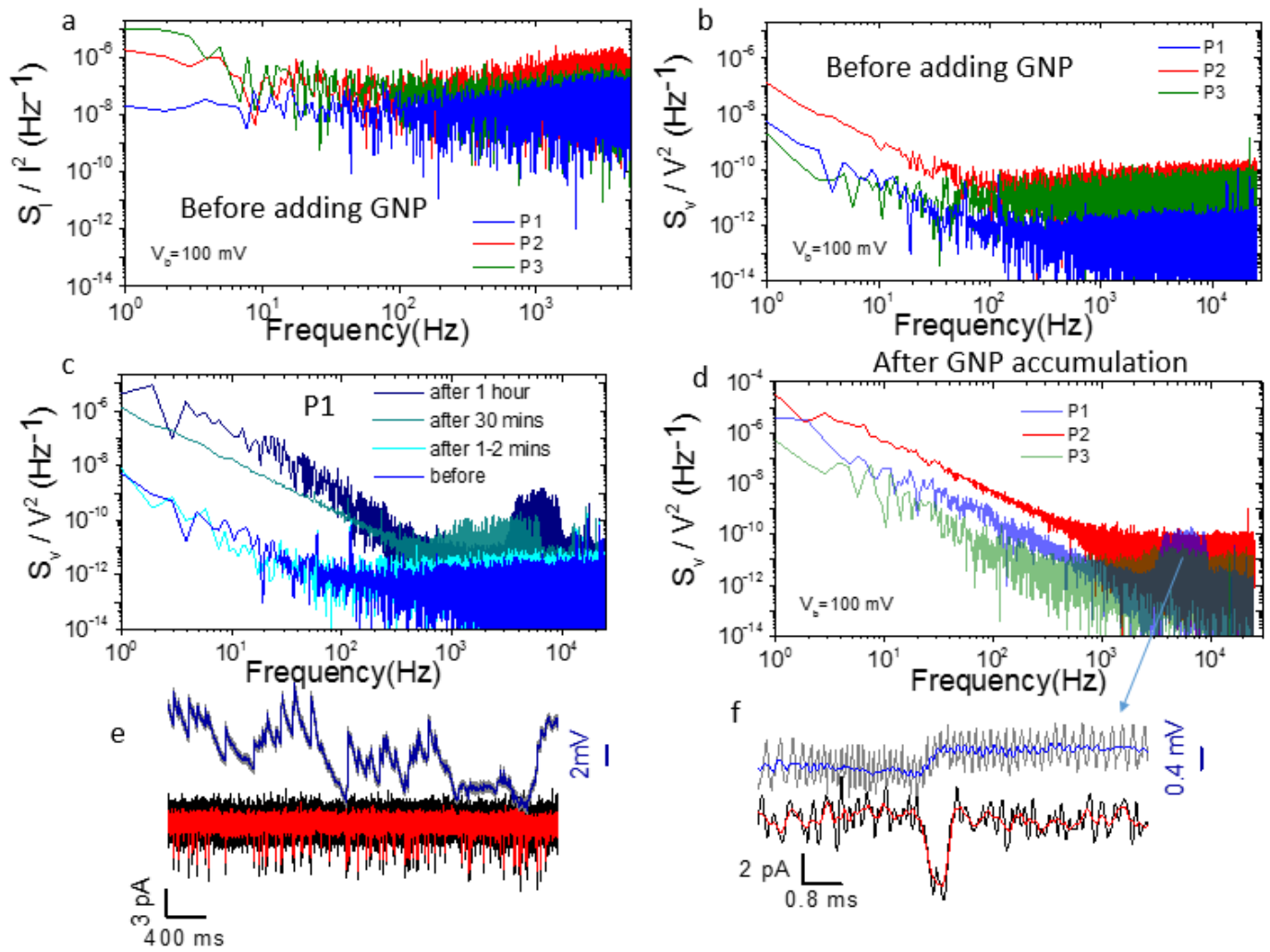

Figure $5.11(a, b)$ The normalized noise power spectra for ionic current (a) and potential (b) for a 1 second time trace from nanopipettes P1, P2 and P3 at applied bias $\mathrm{V}_{\mathrm{b}}=100 \mathrm{mV}$. No GNPs were added in the solution. (c) The normalized potential noise power spectra for P1 before (blue) and after adding GNPs in the solution at various times. A higher bias $\left(\mathrm{V}_{\mathrm{b}}\right.$ $=300 \mathrm{mV}$ ) is used during GNP accumulation. (d) The normalized potential noise spectra for P1, P2 and P3 at $\mathrm{V}_{\mathrm{b}}=100 \mathrm{mV}$ after GNP accumulation. (e) A typical curve of P1 at $50 \mathrm{mV}$ after adding GNPs. The black curve is the raw data for current measurement and the red curve is the smoothed curve of current after 10 points moving average. Similarly, the gray curve is the raw data for potential measurement and the blue curve is the smoothed curve of potential after 10 points moving average. (f) The zoomed in current and potential traces after applying moving average smoothing method. Both raw data and smoothed data are shown. The sampling rate is $50 \mathrm{kS} / \mathrm{s}$ for both measurements and the bandwidth is $5 \mathrm{k} \mathrm{Hz}$ for current and $40 \mathrm{k} \mathrm{Hz}$ for potential. The bath solution is always $10 \mathrm{mM}$ PBS.

\subsection{5: Finite Element Based Numerical Simulations}

We have carried out FEM simulations to understand the potential detection mechanism through the nanopore/nanoelectrode system. We have qualitatively explained the potential detection mechanism in above section. Now we will discuss FEM simulation results in detail. 
Finite element based numerical simulations is used to solve the problems that cannot be solved using analytical solutions. The NP translocation through a single nanopore nanopipette has been thoroughly simulated previously based on a finite element based numerical simulations. ${ }^{20,} 21,25,29$ However, quantitative prediction of the local potential change during translocation of charged nanoparticle through the nanopore of multifunctional nanopipette (nanopore/nanoelectrode) has not been done yet. Therefore, the potential detection by carbon nanoelectrodes (CNE) during the GNP translocation through the nanopore of nanopore/CNE was focused. How the ion fluxes, potential distribution, and electric field within the nanopore affects the local potential change on the CNE during translocation of charged GNP was computed by using coupled PoissonNernst-Planck equation. ${ }^{37-39}$ The nature of ion fluxes and potential distribution are modeled by the Nernst-Planck equation as shown in Equation 5.2 assuming electroneutrality and no convection where the relationship of ion concentrations with electric potential is simulated by Poisson's equation as shown in equation 5.3.

$\mathrm{J}_{\mathrm{i}}=-\mathrm{D}_{\mathrm{i}} \nabla \mathrm{c}_{\mathrm{i}}-\frac{\mathrm{z}_{\mathrm{i}} \mathrm{F}}{\mathrm{RT}} \mathrm{D}_{\mathrm{i}} \mathrm{C}_{\mathrm{i}} \nabla \emptyset$

$\nabla^{2} \emptyset=-\frac{\mathrm{F}}{\epsilon} \sum_{\mathrm{i}} \mathrm{z}_{\mathrm{i}} \mathrm{c}_{\mathrm{i}}$

In above equations, Ji, Di, ci, zi, and $\epsilon$ are, respectively, the ion flux vector, diffusion coefficient, concentration, charge of species i and a dielectric constant in the electrolyte solution. $\Phi$ is the local electric potential and F, R, T and are Faraday's constant, the gas constant, and temperature, respectively.

Multifunctional nanopipette/CNE was modeled using 2D axial symmetric geometry as shown in Figure 5.12 The quasi 3D view near the tip was shown in the inset 
(i) and the CNE displayed a donut shape. This geometry exaggerated the CNE size. As we will discuss later, the size of CNE did not play an important role though it will slightly affect the access ionic resistance and a number of induced charges. Therefore, we still use this geometry because the computation time is much shorter. The half cone angle $\theta$ of the nanopipette was fixed at $6.5^{\circ} .-5 \mathrm{mC} / \mathrm{m}^{2}$ surface charge density was typically applied on the quartz walls if not mentioned otherwise. The nanopipette surface charge effect to the ionic current and potential changes was also compared. The changes are very small, and there are about $7 \%$ increases for both current and potential when the surface charge density of the quartz wall is increased from 0 to $-5 \mathrm{mC} / \mathrm{m}^{2}$.

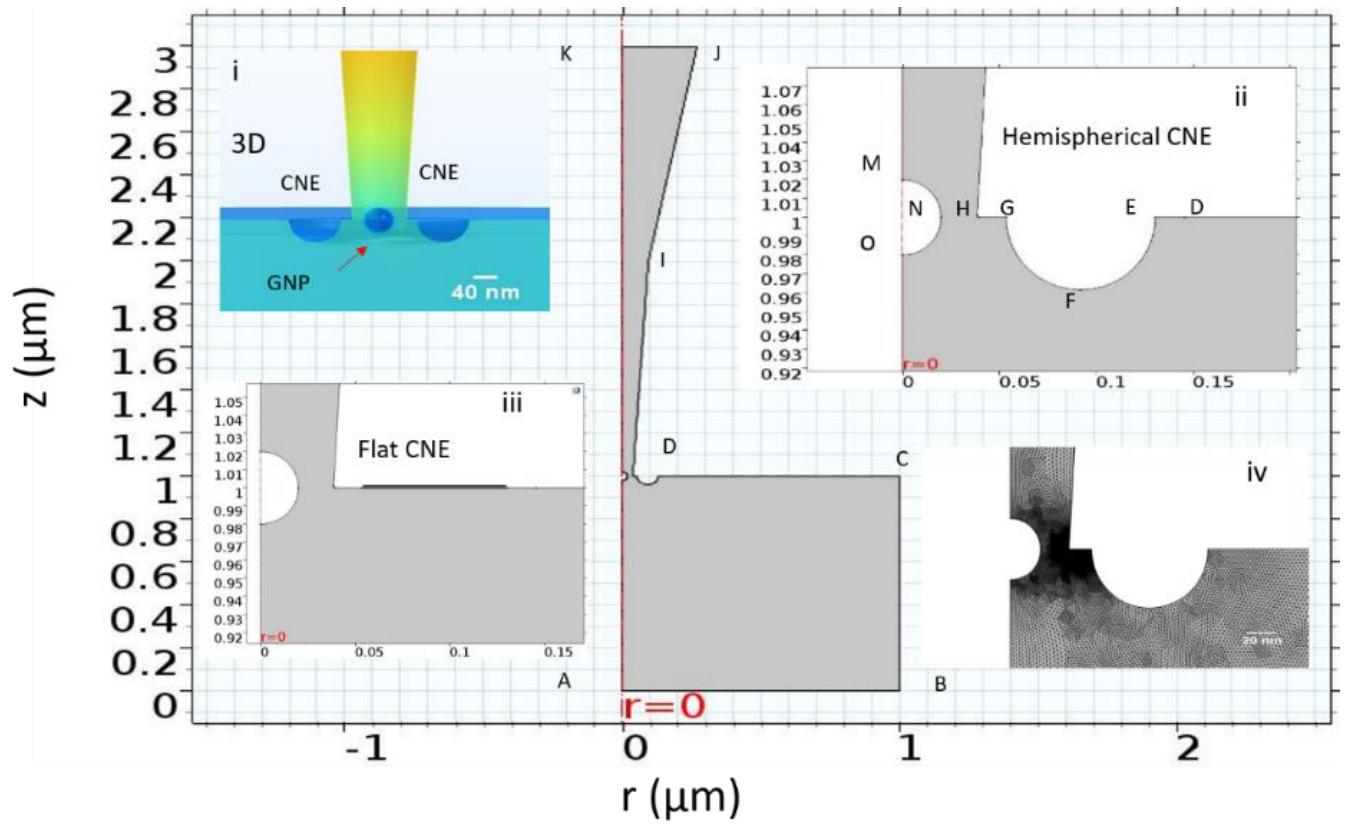

Figure 5.12. The 2D axial symmetric geometry of the nanopipette/CNE used for the FEM simulations. The figures are drawn to scale ( $\mathrm{r}=0$ indicates the axis symmetry line). Insets: (i) the quasi 3D view of the simulation model near the tip. (ii-iii) zoom-in views of the tip region with (ii) a hemispherical shape $\mathrm{CNE}$ with radius $38.5 \mathrm{~nm}$ and (iii) a flat $\mathrm{CNE}$. (iv) mesh distribution near the tip of hemispherical shape CNE.

Similar to experimental conditions, the nanopore diameter and the CNE base size were fixed at $77 \mathrm{~nm}$. The protrusion length of CNE was changed from $38.5 \mathrm{~nm}$ (ii, 
hemispherical CNE) to $0 \mathrm{~nm}$ (iii, flat CNE) in the models of Figure 5.12. The surface area of CNE (ii) is 1.53 time CNE (iii). The simulation was carried out with one $40 \mathrm{~nm}$ GNP or a cluster of three GNPs ( $1 \mathrm{~nm}$ inter-GNP distance) in the model. A surface charge density $-24 \mathrm{mC} / \mathrm{m}^{2}$ was typically applied to the GNP surface if not mentioned otherwise. The surfaces at the CNE and the GNP were allowed to float. A constant potential difference $0.1 \mathrm{~V}$ was applied to the system, and the bath solution is grounded. The whole computation domain was discretized into free triangular elements. The mesh size is much smaller than the Debye length $(\sim 2.8 \mathrm{~nm})$. To simplify the simulation, only two ions, potassium and chloride ions, are used at $10 \mathrm{mM}$ concentration. The parameters used during simulations are listed in Table 5.1.

Table 5.1: Simulations parameters

\begin{tabular}{|l|l|}
\hline Parameters & Value \\
\hline Relative permittivity $\left(\varepsilon_{\mathrm{r}}\right)$ & 80 \\
\hline Temperature $(\mathrm{T})$ & $298 \mathrm{~K}$ \\
\hline Diffusion coefficient $\left(\mathrm{K}^{+}\right)$ & $1.957 \times 10-9\left(\mathrm{~m}^{2} \mathrm{~s}^{-1}\right)$ \\
\hline Diffusion coefficient $\left(\mathrm{Cl}^{-}\right)$ & $2.032 \times 10-9\left(\mathrm{~m}^{2} \mathrm{~s}^{-1}\right)$ \\
\hline Charge number $\left(\mathrm{Z}_{\mathrm{K}}^{+}\right)$ & 1 \\
\hline Charge number $\left(\mathrm{Z}_{\mathrm{Cl}}{ }^{-}\right)$ & -1 \\
\hline Concentration $\left(\mathrm{C}_{\mathrm{K}}{ }^{+}\right)$ & $0.01 \mathrm{M}$ \\
\hline Concentration $\left(\mathrm{C}_{\mathrm{Cl}}{ }^{-}\right)$ & $0.01 \mathrm{M}$ \\
\hline Maximum element (mesh) size & $0.01111 \mu \mathrm{m}$ \\
\hline Minimum element (mesh) size & $0.00008 \mu \mathrm{m}$ \\
\hline Maximum element growth rate & 1.2 \\
\hline Resolution of curvature & 0.3 \\
\hline Resolution of narrow regions & 2 \\
\hline Number of refinements & 3 \\
\hline
\end{tabular}


The boundary conditions and the related physics equations are summarized in Table 5.2

Table 5.2: Typical Boundary Conditions and Related Physics Equations

\begin{tabular}{|l|l|l|}
\hline Surface & Poisson's Equation & Nernst- Plank Equation \\
\hline KA & Axial symmetry & Axial symmetry \\
\hline AB $(\mathrm{Ag} / \mathrm{AgCl}$ electrode) & Ground & Constant concentration \\
\hline BC & Zero Charge & No flux (insulation) \\
\hline CD & Zero Charge & No flux (insulation) \\
\hline DE (quartz) & $-5 \mathrm{mC} / \mathrm{m}^{2}$ or 0 & No flux (insulation) \\
\hline EFG (CNE surface) & $\begin{array}{l}\text { No charge, floating } \\
\text { potential }\end{array}$ & No flux (insulation) \\
\hline GH (quartz) & $-5 \mathrm{mC} / \mathrm{m}^{2}$ or 0 & No flux (insulation) \\
\hline HI (quartz) & $-5 \mathrm{mC} / \mathrm{m}^{2}$ or 0 & No flux (insulation) \\
\hline IJ (quartz) & Zero Charge & No flux (insulation) \\
\hline JK (Ag/AgCl electrode) & $0.1 \mathrm{~V}$ Constant concentration \\
\hline MNO (GNP surface) & $\begin{array}{l}-24 ~ m C / m^{2} \quad \text { (or others), } \\
\text { floating potential }\end{array}$ & No flux (insulation) \\
\hline
\end{tabular}

Following previous works 25,39 , we made the following simplifications in our simulation. 1) we ignored the finite size of ion and water molecule; 2) we ignored the contribution of electroosmosis; 3) we used the 2D axial symmetry in the model geometry, and the 3D geometry is not exactly same as the experiments; 4) the simulation is only for a steady state. With these simplifications, the simulation is less accurate quantitatively. However, the simulation results still capture the main characteristics of the potential changes in the experiments and help us to understand the potential sensing mechanism of the nanopore/CNE nanopipette. Figure 5.13a shows the distribution and direction of the electric field near the nanopipette tip when a GNP is at the nanopore mouth. It also shows the typical geometry with 2D axial symmetry for the FEM simulation. The nanopore orifice is at $\mathrm{z}=1 \mu \mathrm{m}$, and the CNE is nearby. The position of GNP varied along the $\mathrm{z}$-axis. The simulation results revealed that the translocation of a charged GNP through the nanopore altered the local distributions of ion, electric field, and potential near the nanopipette tip, 
as well as the polarization of the floating CNE and GNP. Figure 5.13b shows the distributions of potential and the $\mathrm{z}$-axis component of the electric field (Ez) along the yellow dash line in Figure 5.13a when the GNP is at five different positions along the zaxis. The potential is zero in the bath solution and gradually reaches $0.1 \mathrm{~V}$ deep inside the nanopipette barrel. The zoom-in Figure in the potential plot shows the small potential change induced by the GNP. Obviously, the negative charged GNP lowers the local potential. The Ez reaches the negative maximum at the nanopore mouth. The positive spike at $\mathrm{Z}=1 \mu \mathrm{m}$ is caused by the surface charge at the quartz surface. The bipolar shape spikes (indicated by red arrows) in Ez curves are due to the corresponding local potential disturbance by the charged GNP showing in the potential plot. Figure 5.13c shows the typical ionic current (black color) and potential (red color) changes as a function of the GNP position. The simulated potential dip reminds us the experimental results from single GNP in Figure 5.4a. The current spike and the potential dip showed the same duration time. Similar to the trace (iii) of Figure 5.4 a, we labeled step number 1, 2 and 3 in the simulated potential dip. Because of the small conical angle of the nanopipette, the current and potential gradually return to the original levels at step 3 even the GNP is several hundred nanometers inside the nanopipette. As we discussed, there are two sensing mechanisms contribute to the potential sensing at the CNE. In Figure 5.13d, we showed the shape evolution of the potential dip when the magnitude of GNP surface charge density is increased from zero. With no charge at the GNP surface, the potential dip at the CNE is bipolar, showing the major contribution from the voltage divider model. With the increase in the surface charge density at the GNP surface, the initial potential increase, indicated by a blue arrow in Figure 5.13d, is gradually reduced until fully disappears. This confirms that 
the charge sensing mechanism gradually takes over with the increase of GNP surface charge at step 1. In addition to the fixed charges, the induced charges at the CNE also compete with the voltage divider model and suppresses the access resistance increase which has caused an initial potential increase (see Figure 5.14c). Figure 5.13d also suggests the magnitude of the potential dip correlates with the magnitude of the GNP surface charge density. We further tested the CNE geometry effect by changing the area and shape of the CNE. The ionic current change is negligible as long as the CNE is not too big to effectively block the ionic pathways. The potential change only increased about 5\% when the CNE area increased 1.53 times. This explains why we obtained reproducible potential changes in experiments while the fabricated CNE area changed significantly. In addition, we found the CNE can measure a bigger potential change when several GNPs near the nanopore entrance (see Figure 5.14f). 

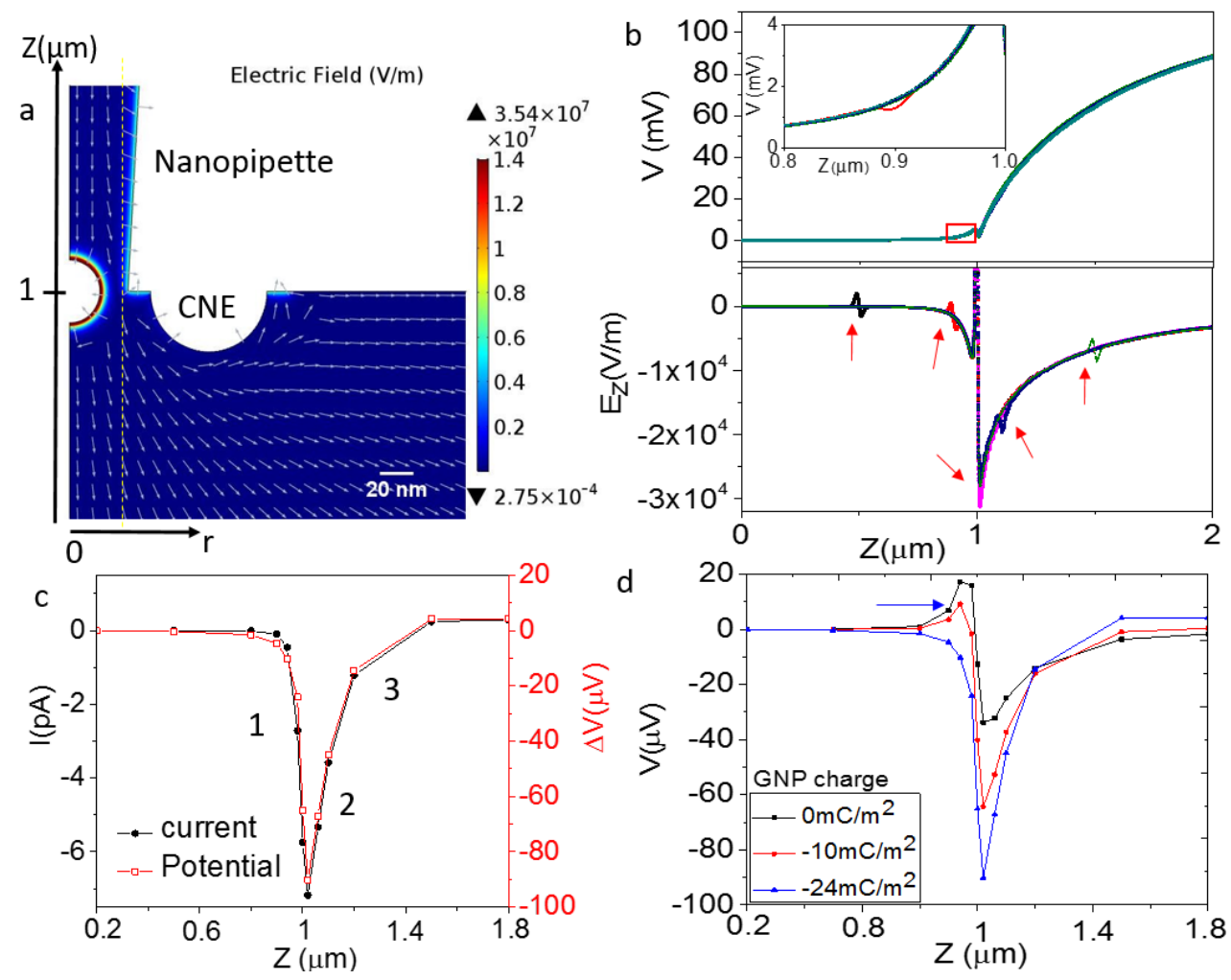

Figure 5.13 FEM simulation results (a) The Electric field distribution near the nanopipette tip when a GNP is at the nanopore orifice $(\mathrm{Z}=1 \mu \mathrm{m}, \mathrm{r}=0 \mathrm{~nm})$. The white arrows indicate the direction of the field, and the color bar shows the intensity of the field. The axis symmetry line is at $r=0$. (b) The distributions of potential (top) and $\mathrm{Z}$ component electric field $(E z=d V / d Z)$ (bottom) along the yellow dash line showing in (a). Both plots contain five distributions with a GNP at five different locations (indicated by the red arrows). The inset in potential distribution shows the small change of the potential distribution when the GNP is near the nanopore entrance $(\mathrm{Z}=0.9 \mu \mathrm{m}, \mathrm{r}=0 \mathrm{~nm})$. (c) The ionic current (black) changes of the nanopore and potential (red) change at the CNE as a function of GNP center position in $\mathrm{Z}$ direction along the axis symmetry line. (d) The evolution of the $\Delta \mathrm{V}-\mathrm{Z}$ plots as the surface charge density of the GNP increase. The surface charge densities at the surface of quartz, CNE and GNP are $-5 \mathrm{mC} / \mathrm{m}^{2}, 0 \mathrm{mC} / \mathrm{m}^{2}$ and $-24 \mathrm{mC} / \mathrm{m}^{2}$ respectively, if not mentioned otherwise.

Figures $5.14(\mathrm{a}, \mathrm{b})$ showed the electric potential and net ion distribution near the nanopipette tip with the presence of a GNP at the nanopore mouth. The polarization of CNE was revealed in the net ion distribution (Figure 5.14(b)) and negative induced charges were near the nanopore entrance. The results in Figure 5.14(c) demonstrated how the induced surface charges at the CNE and GNP surfaces affected the potential change at the 
CNE. Without fixed and induced charges at nearby surfaces, the voltage divider model dominates, and an obvious potential increase is observed when the GNP is close to the nanopore entrance. After allowing the CNE to be polarized, the increased negative induced charges at the CNE compete with the increased access ionic resistance while the neutral GNP approaches the nanopore. The increase of induced charges at the CNE is due to the increase of the local electric field with the approaching of the GNP to the nanopore. The polarization of GNP shows a negligible effect on the potential change at the CNE. Figure 5.14(d) shows the effect of the CNE geometry to the potential measurements. As shown in the inset, the protruded CNE can detect the potential change slightly earlier with a bigger magnitude. However, the difference is only about $5 \%$ when we changed the CNE area 1.53 times. Figure 5.14 (f) compares the potential changes $\Delta \mathrm{V}$ when one and three GNPs near the nanopore mouth. It shows that the CNE can cumulatively measure a bigger potential change from three charged GNPs near the CNE ( $\sim 25 \%$ change). The potential increase near $\mathrm{Z}=1 \mu \mathrm{m}$ in the red plot is attributed to the increased access resistance by the accumulation of 3 GNPs near the nanopore. This is somewhat exaggerated by the donut shape of the CNE. 

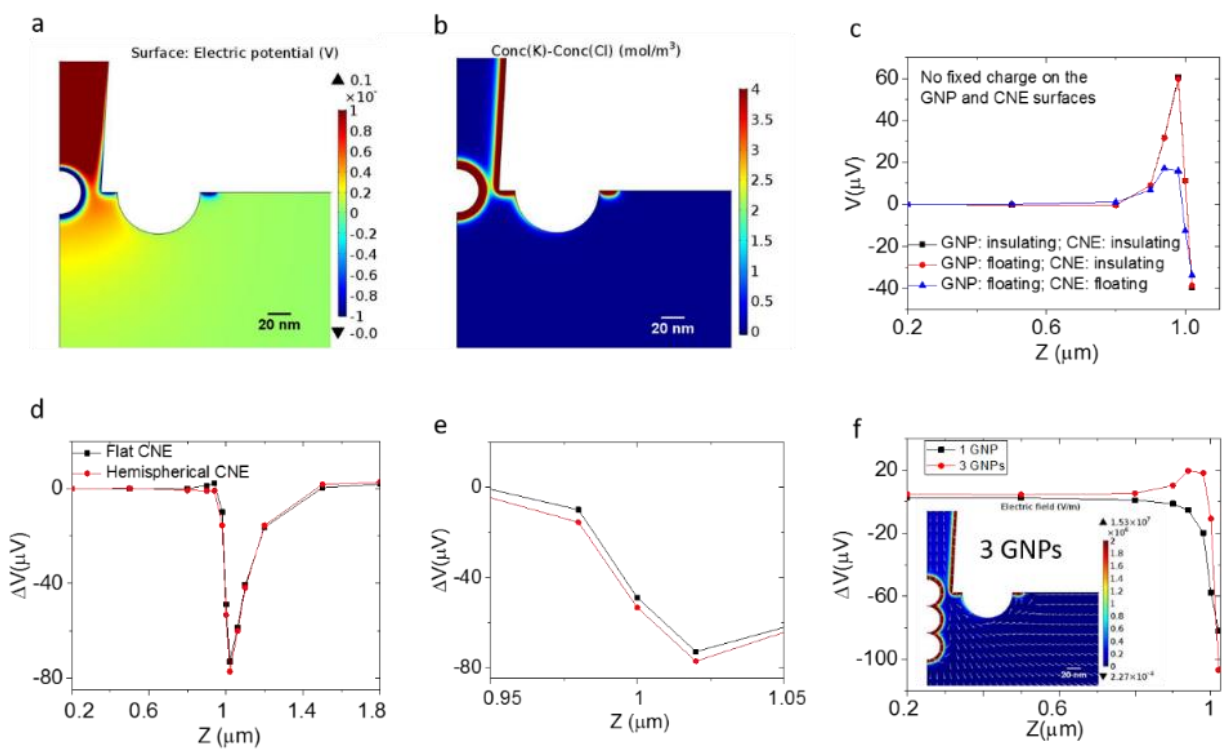

Figure 5.14. (a) Electric potential distribution near the tip region. (b) The net ion distribution near the tip region. Only potassium and chlorine ions are considered in the simulation. (c) The potential changes versus the GNP center position along $\mathrm{Z}$ axis at different GNP and CNE polarization conditions. (d) The potential changes versus the GNP center position along $\mathrm{Z}$ axis with CNE geometry (ii) and (iii) in (6.1). (e) The zoom-in of (d) near the nanopore mouth. (f) The potential changes versus the GNP center position along $\mathrm{Z}$ axis with one (black) and three GNPs (red) at the nanopore mouth. For 3 GNPs case, the $\mathrm{Z}$ position is the center of the first GNP. The inset is the electric field distribution for 3 GNPs.

\section{3: Conclusions}

In summary, we have fabricated the nanopore/nanoelectrode multifunctional nanopipette by an economic, facile and quick method. The fabricated CNE coupled very well with the nanopore at the nanopipette tip. Using $40 \mathrm{~nm}$ GNPs as the model particles, we have demonstrated that this type of multifunctional nanopipettes displayed the advantage of both nanopore and nanoelectrode. We measured not only the translocation of individual GNPs through the nanopore, but also the dynamic accumulation of several GNPs outside the nanopore. The resistive-pulse sensing method by nanopore is highly sensitive to individual GNP translocation event, but the major change is limited to the narrowest 
region of the nanopore. In contrast, the potential sensing mode by the protruded $\mathrm{CNE}$ covers a bigger spatial range and can cumulatively detect multiple GNPs outside the nanopore. In the experiments, the GNPs accumulate outside the nanopore and form clusters. The CNE can detect the GNP cluster formation outside the nanopore while the nanopore cannot. However, the CNE alone cannot measure GNP individually. Meanwhile, the nanopore drives one GNP at a time from a GNP assembly to enter the nanopore. This enables the CNE to measure the potential from just one GNP in a crowded environment with many GNPs. The potential method also shows much lower noise at high frequency and therefore is capable of measuring faster translocation events than the resistive-pulse sensing method. We also compared the potential detection performance between dualnanopore nanopipette and $\mathrm{CNE} /$ nanopore nanopipette. The control results confirmed the advantage of integrating $\mathrm{CNE}$ and nanopore for the potential sensing. The experimental results were also well-explained by FEM simulations.

This chapter demonstrated the advantages of multifunctional nanopipette and multimode sensing. The nanopore/nanoelectrode nanopipette is not limited to GNP analysis but is also suitable for the label-free analysis of protein, virus and various biological and synthetic NPs. Dual-nanopore nanopipette has been used for potential/surface charge imaging of live cell surface. ${ }^{36}$ Because the nanopore/CNE nanopipette showed better sensitivity to surface potential/charge, we expect that we can use it as a scanning probe for potential/charge imaging of cell surface in the electrolyte. Currently, the high precision quantitative measurements are limited by the large variations in the geometry of multifunctional nanopiepttes. The development of better characterization methods for multifunctional nanopipettes ${ }^{20}$ will enable more quantitative 
measurements of NPs. Furthermore, the hybrid potential detection mechanism developed in this work is also applicable to other micro-/nano-fabricated nanopore/nanoelectrode devices.

Based on the numerical simulation results, potential sensing mechanism of the floating carbon electrode was found to be very sensitive to the surface charge of the GNP near the nanopore. In summary, we found that 1) the translocation of individual GNP can induce downward potential drop at the floating $\mathrm{CNE}$ and downward ionic current spike at the nanopore; 2) the protrusion of CNE leads to an early potential detection for the arrival of GNP and can expand the potential sensing zone; 3) the increased number of GNPs near the protruded CNE induces a bigger potential drop at the CNE. The numerical simulation results are useful not only to validate experimental nanopore/CNE results but also very helpful in the understanding of fundamental charge sensing mechanism of the CNE.

\section{4: References}

1. Panday, N., Qian, G., Wang, X., Chang, S., Pandey, P. \& He, J. ACS Nano 2016, $10,11237$.

2. Xie, P., Xiong, Q., Fang, Y., Qing, Q. \& Lieber, C.M. Nature nanotechnology 2012, $7,119$.

3. Song, W., Pang, P., He, J. \& Lindsay, S. ACS Nano 2013, 7, 689.

4. Larkin, J., Foquet, M., Turner, S.W., Korlach, J. \& Wanunu, M. Nano Letters 2014, $14,6023$.

5. Cecchini, M.P., Wiener, A., Turek, V.A., Chon, H., Lee, S., Ivanov, A.P., McComb, D.W., Choo, J., Albrecht, T., Maier, S.A. \& Edel, J.B. Nano Letters 2013, 13, 4602 .

6. Fosdick, S.E., Anderson, M.J., Nettleton, E.G. \& Crooks, R.M. Journal of the American Chemical Society 2013, 135, 5994. 
7. Freedman, K.J., Otto, L.M., Ivanov, A.P., Barik, A., Oh, S.-H. \& Edel, J.B. Nature communications 2016, 7.

8. Wang, Y., Kececi, K., Mirkin, M.V., Mani, V., Sardesai, N. \& Rusling, J.F. Chemical Science 2013, 4, 655.

9. Kleijn, S.E.F., Lai, S.C.S., Miller, T.S., Yanson, A.I., Koper, M.T.M. \& Unwin, P.R. J. Am. Chem. Soc. 2012, 134, 18558.

10. Morris, C.A., Friedman, A.K. \& Baker, L.A. Analyst 2010, 135, 2190.

11. Hu, K., Wang, Y., Cai, H., Mirkin, M.V., Gao, Y., Friedman, G. \& Gogotsi, Y. Analytical Chemistry 2014, 86, 8897.

12. Takahashi, Y., Shevchuk, A.I., Novak, P., Murakami, Y., Shiku, H., Korchev, Y.E. \& Matsue, T. Journal of the American Chemical Society 2010, 132, 10118.

13. Takahashi, Y., Shevchuk, A.I., Novak, P., Zhang, Y., Ebejer, N., Macpherson, J.V., Unwin, P.R., Pollard, A.J., Roy, D., Clifford, C.A., Shiku, H., Matsue, T., Klenerman, D. \& Korchev, Y.E. Angewandte Chemie International Edition 2011, 50,9638 .

14. Takahashi, Y., Shevchuk, A.I., Novak, P., Babakinejad, B., Macpherson, J., Unwin, P.R., Shiku, H., Gorelik, J., Klenerman, D., Korchev, Y.E. \& Matsue, T. Proc. Natl. Acad. Sci. U.S.A. 2012, 109, 11540.

15. Lai, S.C.S., Dudin, P.V., Macpherson, J.V. \& Unwin, P.R. Journal of the American Chemical Society 2011, 133, 10744.

16. Morris, C.A., Chen, C.-C. \& Baker, L.A. Analyst 2012, 137, 2933.

17. Derylo, M.A., Morton, K.C. \& Baker, L.A. Langmuir 2011, 27, 13925.

18. Walsh, D.A., Fernández, J.L., Mauzeroll, J. \& Bard, A.J. Analytical Chemistry $2005,77,5182$.

19. Chen, C.-C., Zhou, Y., Morris, C.A., Hou, J. \& Baker, L.A. Analytical chemistry 2013, 85, 3621 .

20. Perry, D., Momotenko, D., Lazenby, R.A., Kang, M. \& Unwin, P.R. Analytical Chemistry 2016, 88, 5523.

21. Tiwari, P.B., Astudillo, L., Miksovska, J., Wang, X., Li, W., Darici, Y. \& He, J. Nanoscale 2014, 6, 10255. 
22. Vlassiouk, I., Smirnov, S. \& Siwy, Z. Nano Letters 2008, 8, 1978.

23. White, H.S. \& Bund, A. Langmuir 2008, 24, 2212.

24. Vilozny, B., Actis, P., Seger, R.A., Vallmajo-Martin, Q. \& Pourmand, N. Anal. Chem. 2011, 83, 6121.

25. Lan, W.-J., Holden, D.A., Zhang, B. \& White, H.S. Analytical Chemistry 2011, 83, 3840 .

26. German, S.R., Luo, L., White, H.S. \& Mega, T.L. The Journal of Physical Chemistry C 2012, 117, 703.

27. Wang, Y., Cai, H. \& Mirkin, M.V. ChemElectroChem 2015, 2, 343.

28. Weatherall, E. \& Willmott, G.R. J. Phy. Chem. B 2015, 119, 5328.

29. Lan, W.-J., Kubeil, C., Xiong, J.-W., Bund, A. \& White, H.S. The Journal of Physical Chemistry C 2014, 118, 2726.

30. Liu, H., He, J., Tang, J., Liu, H., Pang, P., Cao, D., Krstic, P., Joseph, S., Lindsay, S. \& Nuckolls, C. Science 2010, 327, 64.

31. Krishnakumar, P., Gyarfas, B., Song, W., Sen, S., Zhang, P., Krstic, P. \& Lindsay, S. ACS nano 2013, 7, 10319.

32. Venta, K.E., Zanjani, M.B., Ye, X., Danda, G., Murray, C.B., Lukes, J.R. \& Drndić, M. Nano Letters 2014, 14, 5358.

33. Cheon, D., Kumar, S. \& Kim, G.-H. Applied Physics Letters 2010, 96, 013101.

34. Hermanson, K.D., Lumsdon, S.O., Williams, J.P., Kaler, E.W. \& Velev, O.D. Science 2001, 294, 1082.

35. Gierhart, B.C., Howitt, D.G., Chen, S.J., Smith, R.L. \& Collins, S.D. Langmuir 2007, 23, 12450.

36. Zhou, Y., Chen, C.-C., Weber, A.E., Zhou, L. \& Baker, L.A. Langmuir 2014, 30, 5669.

37. Perry, D., Al Botros, R., Momotenko, D., Kinnear, S.L. \& Unwin, P.R. ACS Nano 2015, 9, 7266. 
38. Perry, D., Paulose Nadappuram, B., Momotenko, D., Voyias, P.D., Page, A., Tripathi, G., Frenguelli, B.G. \& Unwin, P.R. Journal of the American Chemical Society 2016, 138, 3152 .

39. Snowden, M.E., Güell, A.G., Lai, S.C.S., McKelvey, K., Ebejer, N., O’Connell, M.A., Colburn, A.W. \& Unwin, P.R. Analytical Chemistry 2012, 84, 2483. 


\section{CHAPTER 6: MEASURING EXTRACELLULAR POTENTIAL DISTRIBUTIONS OF A SINGLE LIVING CELL MEMBRANES BY SICM}

Chapter 6 presents the integration of commercially available SICM into the multifunctional SICM and use it for quantitative measurement of extracellular membrane potential distribution of a single living cell membrane. Some of the contents of this chapter have been adapted from the manuscript, which is being prepared for publication.

\section{1: Introduction}

Recently, the existence of multiple microdomains of membrane potential around individual cells has been explored by voltage reporter dye using fluorescence microscopy. ${ }^{1}$

${ }^{2}$ All cells, not only excitable neurons and muscles, exhibits multiple microdomains of extracellular membrane potential. ${ }^{3}$ These microdomains of membrane potential are induced by the systematically distributed ion channels and pumps in living cell membrane. The activities of ion channels and pumps will locally modify the ion or chemical concentrations and membrane potential regionally. The distribution and dynamics of all the ion channels will lead to physiological micro-domains around the individual living cell and a cluster of cells. One hypothesis is that these long lasting membrane potential patterns play instructive roles for embryonic patterning, regenerative repair, and suppression of cancerous disorganization, wound healing. ${ }^{4-6}$ To validate this hypothesis, it is very important to acquire the details of these micro-domains of membrane potential with high spatial resolution in a long period. As compared to the voltage reporter dye based fluorescence microscope method, SICM can achieve quantitative potential measurements of non-labeled living cell membranes with higher spatial resolution. 
Scanning ion conductance microscopy (SICM) is a new scanning probe microscopy (SPM) technique for living cell imaging and analysis. ${ }^{7,}{ }^{8}$ In comparison to other SPM techniques, SICM has robust feedback mechanism which is necessary to control the pipette position above the sample precisely. Because of its robust feedback mechanism, SICM has several diverse advantages for biological applications. ${ }^{9-12}$ SICM can generate topography images of living cells as well as fixed cells with high resolution under physiological conditions. It not only can take topography images of the biological samples but also can provide supplementary information about samples like surface charge distribution, electrochemical activities, ion channel distributions. ${ }^{13-15}$ However, development of SICM for mapping of topography and extracellular potential distribution of the single living cell still is in primitive phase. Recently, Unwin's group has reported simultaneously mapping of both the topography and surface charge of living Zea mays root hair cells with bias modulated SICM, which is still not the direct mapping of surface charge. ${ }^{14}$ They recorded the current images of the root hair cell and converted it to the surface charge map with the help of finite element simulations. Klausen et.al revealed the capability of SICM for mapping of surface charge density of lipid bilayers with DC mode SICM however it was not a direct measurement of the surface charge density of the lipid bilayers. They took the topography images of the same lipid membrane at positive and negative applied bias and subtracted these two images to differentiate the surface charge density. ${ }^{16}$ Baker's group have successfully developed the potentiometric SICM for direct potential imaging of synthetic nanopore, however, simultaneously mapping of topography and the potential image has not reported yet. ${ }^{17}$ Even if many research group have demonstrated the capability of the SICM for potential distribution measurement of the living cell, directly mapping of 
topography and potential distribution of a living cell membrane remained as a challenging task. We have utilized the SICM technique for simultaneously mapping of the topography and potential distributions of the single living cell membrane. Furthermore, we have measured the extracellular membrane potential distribution of living B16 and Mela-A cells quantitatively with SICM.

\section{2: Methods}

Detailed instrumentation and operational mechanism of multifunctional SICM for potential measurement are given in chapter 2 under section 2.4. Multifunctional-SICM makes use of a double-barrel theta nanopipette as a probe, where probe position is controlled by the current measured in one barrel and the potential is measured in a second barrel.

\subsection{1: Cell Culture on PDMS}

The B16-F10 mouse melanoma cell line (ATCC, CRL-6322) was cultured in Dulbecco's Modified Eagle's Medium (DMEM) supplemented with 10\% Fetal Bovine Serum, $100 \mathrm{U} / \mathrm{ml}$ penicillin, and $100 \mathrm{U} / \mathrm{ml}$ streptomycin at $37{ }^{\circ} \mathrm{C}$ in $5 \% \mathrm{CO}_{2}$. Melan-A, an immortalized mouse melanocyte cell line (a kind gift of Dr. William J. Pavan, NIH ${ }^{18}$ ), was cultured in RPMI 1640 supplemented with 10\% Fetal Bovine Serum, $100 \mathrm{U} / \mathrm{ml}$ penicillin, $100 \mathrm{U} / \mathrm{ml}$ streptomycin, $200 \mathrm{nM}$ TPA and $200 \mathrm{pM}$ cholera toxin at $37{ }^{\circ} \mathrm{C}$ in $10 \% \mathrm{CO}_{2}$. PDMS was sterilized by autoclave and incubated with Fibronectin (Gibco) at $50 \mu \mathrm{g} / \mathrm{ml}$ in PBS. Allow the fibronectin solution to air dry for at least 45 minutes at room temperature and wash the PDMS with PBS before seeding the cells. Both cells were seeded at a low confluency to allow for single cell potential detection, and dead cells were removed the next day after cell seeding by changing the media. Cells were cultured for at least 36 hours to reach a stable state before any potential experiments. 


\section{3: Results and Discussion}

An illustration of the multifunctional SICM experimental setup for potential measurement is shown in Figurer 6.1(a). A double barrel theta nanopipette is used as the scanning probe. One barrel connected with the voltage amplifier measures the potential differences while the other barrel connected to the current amplifier measures the distance dependent ion current for positioning of the probe and noninvasive topography imaging of samples in a similar way as single barrel nanopipette. Simultaneously recorded approach curves of current and potential shown in Figure 6.2(a) validated the experimental setup. The equivalent circuit is shown in Figure 6.1(b) explains the potential change as a function of distance based on voltage divider model. Variable resistance $\mathrm{R}_{\mathrm{ac}}$ is connected in series

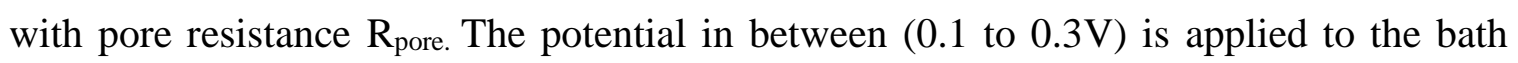
solution whereas the $0 \mathrm{~V}$ (ground) potential is applied inside the barrel connected to the current amplifier. The barrel connected with voltage amplifier is treated as a floating point potential probe and positioned at the location between $\mathrm{R}_{\mathrm{ac}}$ and $\mathrm{R}_{\text {pore. }}$ The approach of the double barrel probe towards the substrate consequentially increases the magnitudes of $\mathrm{R}_{\mathrm{ac}}$. This resistance change alters the potential distribution in the circuit, and therefore the potential at the tip of the potential probe. The potential change as a function of probesample distance validates our potential measurement setup. However, it also suggests that we need to pay attention to the height effect when discussing the measured potential. 

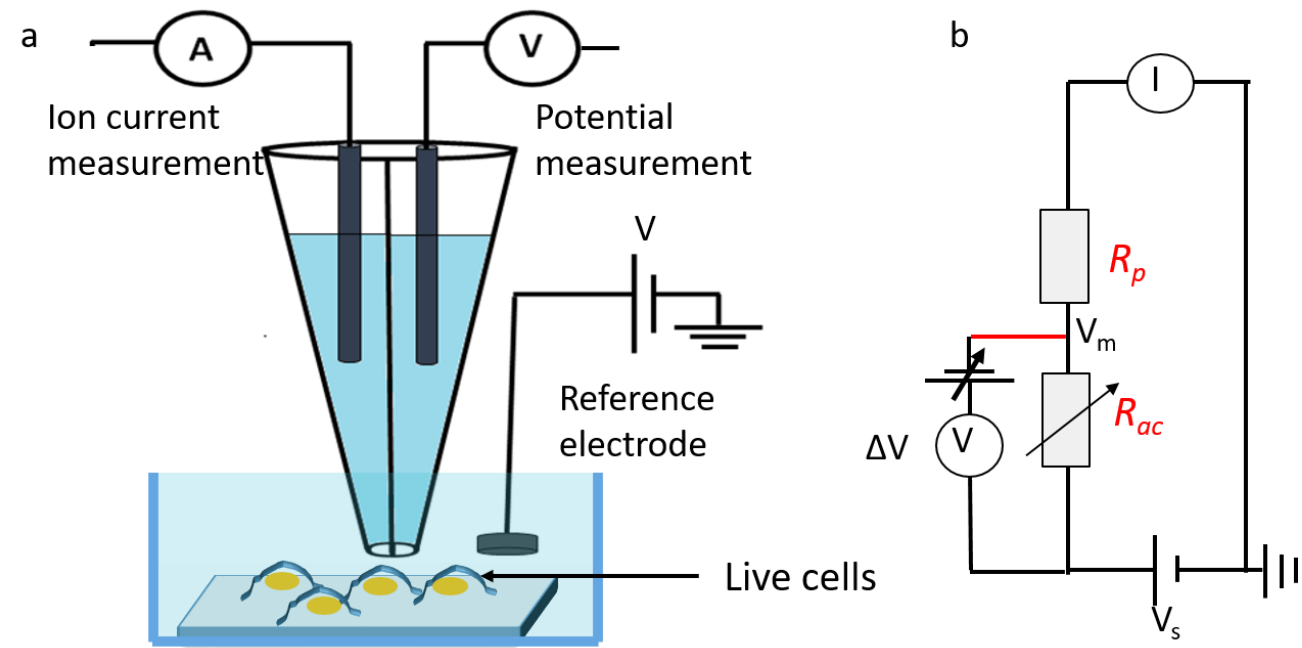

Figure 6.1 (a) Schematic of a potentiometric SICM (P-SICM) setup using the theta nanopipette as a probe. (b) Equivalent circuit model of the experimental setup.

The surface potential is also affected by the surface charge. When the potential probe approaches towards a charged substrate with a distance smaller than the double layer thickness, it can sense the potential due to the surface charge of the substrate. To test the surface charge measurement by the potentiometric SICM, we have measured the potential of the positively charged substrate and the negatively charged substrate with the same pipette in the same condition. Single point potential measurement was performed first. In PBS solution, Lysozyme modified PDMS was used as a positively charged substrate whereas bovine serum albumins (BSA) was used as the negatively charged substrate. The sample was prepared on the graphene modified PDMS substrate. First, PDMS was modified by graphene and graphene modified PDMS was again modified with either lysozyme or BSA. Potential approach/retract curves were collected in 1X PBS electrolyte solution at neutral $\mathrm{pH}$. The distance is about a few $\mathrm{nm}$, which is smaller than the double layer thickness. We used the potential at $20 \mu \mathrm{m}$ away from the surface as the reference 
point, which is the same as the bulk solution potential. We then measured the potential close the surface. The potential difference Vs-Vref is obviously bigger for a negatively charged substrate. Surface charge effect was clearly observed on the potential approach/retract curves acquired from two different charged substrate as shown in Figure 6.2(b). There was electrostatic attraction happened with positively charged substrate whereas electrostatic repulsion occurred with a negatively charged substrate with negatively charged quartz nanopipette. Electrostatic attraction caused the smaller potential detection on positive charge substrate whereas electrostatic repulsion caused bigger potential detection on the negative charge substrate. In our experimental setup, both sensing mechanism plays a role in surface potential/charge measurement. We will explain the potential sensing mechanism more quantitatively with finite element method simulations.

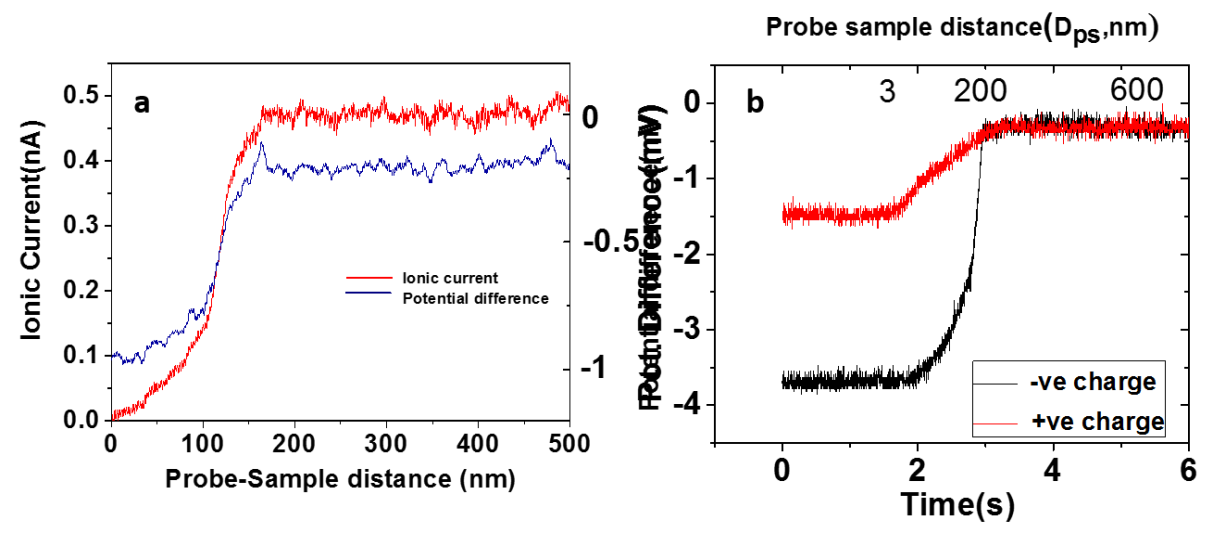

Figure 6.2 (a) simultaneously acquired approach curves for ionic current and the potential difference with potentiometric SICM. (b) Approach /retract curve spectroscopy on positive and negative charged substrate.

After successfully demonstrated the potential measurement capacity of our potentiometric SICM set up, we directly applied it to measure the extracellular potential distribution of the living Melanoma (B16) which is the cancerous skin cell and 
melanocytes (Mela-A) which are the normal skin cell. B16 and Mela-A were chosen because it will help us to compare the extracellular potential distribution between the normal cell and cancerous cell of the same type of cell line. Living cell experiment was performed under the physiological buffer with $37^{\circ}$ temperature. The potential measurement was carried out with two technique, one was single point measurement technique, and the other was directly imaging the potential distribution.

\subsection{1: Single Point Potential Measurement}

During single point measurement, theta nanopipette was approached first just above the center of the single cell of interest by monitoring the ionic current and potential-time $(\mathrm{V}-\mathrm{t})$ and ionic current -time (I-t) traces were recorded at two different height from the cell. One data recording height was 20 micron above from the cell which was set as a reference level, and the other was very close $(\sim 2 \mathrm{~nm})$ above the cell. Then the theta nanopipette was withdrawn few micron ( $\sim 50$ micron) and relocate to another position of the same cell and data were recorded similarly. Four potential data were recorded from each cell around 510 micron periphery from the center of the cell. The I-t traces were collected at applied bias by Femto DE-DLPCA-200_R17 Variable Gain Current Amplifier with $10^{9}$ gain and the V-t traces were measured by a home-built, battery powered high input impedance differential amplifier (based on an instrumentation amplifier) at a 10x gain. A digital oscilloscope (Yokogawa DL850 scopecorder) was used to record the current and potential traces with a sampling rate $500 \mathrm{~Hz}$ and bandwidth $400 \mathrm{~Hz}$.

In the single-point measurement mode, potential changes were monitored at the tip of floating potential probe when the probe was held at a fixed probe-sample distance. Theta nanopipette was approached above the center of a single cell of interest by monitoring ionic 
current. The potential was recorded at two different height from the cell. 20 micron above from the cell was set to a reference level, and the closet probe-sample distance was always fixed around $\sim 2 \mathrm{~nm}$ during fixed point measurement. The actual extracellular potential at a single point of the cell membrane was calculated from the difference of the potential measured at two different height. Four potential data were recorded from each cell around 5-10 micron periphery from the center of the cell. A similar type of the cell was chosen with the help of the oscilloscope for the data collection. Several batches of the sample were used for the data collections. All cells were cultured on the either Fibronectin modified PDMS or collagen modified PDMS.

Fixed point measurement was done in HEPES buffer having total ionic concentration about $151 \mathrm{mM}$ with $45 \mathrm{mM}$ and $5 \mathrm{mM} \mathrm{KCl}$ concentration. The result shown in Figure 6.3 (a) clearly shows the potential difference between Mela-A and B16 cell.

The permeability to $\mathrm{K}^{+}$ion is much higher at rest than $\mathrm{Na}^{+} \mathrm{Cl}^{-}$, and $\mathrm{Ca}^{+}$. Change of extracellular $\mathrm{K}^{+}$concentration can play a dominant role in resting membrane potential. Zhou et. al have reported that the membrane potential of baby hamster kidney (BHK) cells, can be depolarized by increasing the extracellular $\mathrm{K}+$ concentration. ${ }^{19}$ We have carried out experiments to measure extracellular membrane potential distribution of Mela-A and B16 in living cell buffers with different $\mathrm{K}^{+}$concentrations while maintaining the same ionic strength. HEPES buffer containing $\mathrm{NaCl} 103 \mathrm{mM}, \mathrm{KCl} 45 \mathrm{mM}, \mathrm{CaCl} 22.5 \mathrm{mM}, \mathrm{MgCl} 2$ 1.2mM, HEPES $10 \mathrm{mM}$, and Glucose $10 \mathrm{mM}$ with neutral $\mathrm{pH}$ was prepared. For a low $\mathrm{K}^{+}$ solution, $\mathrm{NaCl}$ was replaced with an equal molar concentration of $\mathrm{KCl}$ and other components remained the same. Both the mean magnitude and spatial variation of the extracellular potential of the melanoma cells are bigger than those of melanocytes with the 
outside potassium concentration $45 \mathrm{mM}$. Similar type of measurements was carried out in a different batch of samples for both Mela-A and B16 in HEPES buffer with 5mM potassium concentration and compared the result with the $45 \mathrm{mM}$ outside potassium concentration. As shown in figure 6.3(b) and 6.3(c), the measured extracellular potential was increased when we did the measurement in the $5 \mathrm{mM}$ concentration of the potassium ions in outside bath solution. Furthermore, our result showed extracellular membrane potential of melanocytes (Mela-A) changed more in compare to the melanoma (B16). The mean magnitude of extracellular membrane potential of B16 was not changed significantly whereas it increased almost $600 \mu \mathrm{V}$ in Mela-A cell in $5 \mathrm{mM} \mathrm{KCl}$ compared to $45 \mathrm{mM} \mathrm{KCl}$. In the mammalian cell, the intracellular potassium concentration is approximately 30 times as high as the extracellular concentration $(150$ vs. $5 \mathrm{mM})$. If the outside potassium concentration is made $45 \mathrm{mM}$, the membrane potential becomes more positive, which is called depolarization. In $5 \mathrm{mM}$ in compare to the $45 \mathrm{mM}$ outside $\mathrm{KCl}$ concentration, membrane potential becomes more negative which is called hyperpolarization. Our measurement also showed hyperpolarization behavior in $5 \mathrm{mM} \mathrm{KCl}$ and depolarization behavior in $45 \mathrm{mM} \mathrm{KCl}$ of outside concentration in both Mela-A and B16 cell. We have used the absolute value of the potential measurement in the histograms of figure $6.3(\mathrm{a}, \mathrm{b}$ and c).

Values of extracellular membrane potential are reported as the mean \pm SD of measurements performed on different batches of cell samples with same conditions. The Cohen's d value was calculated to consider the difference between the two mean values statistically significant. The Cohen's d value higher than 0.5 was considered as the statically significant value. 


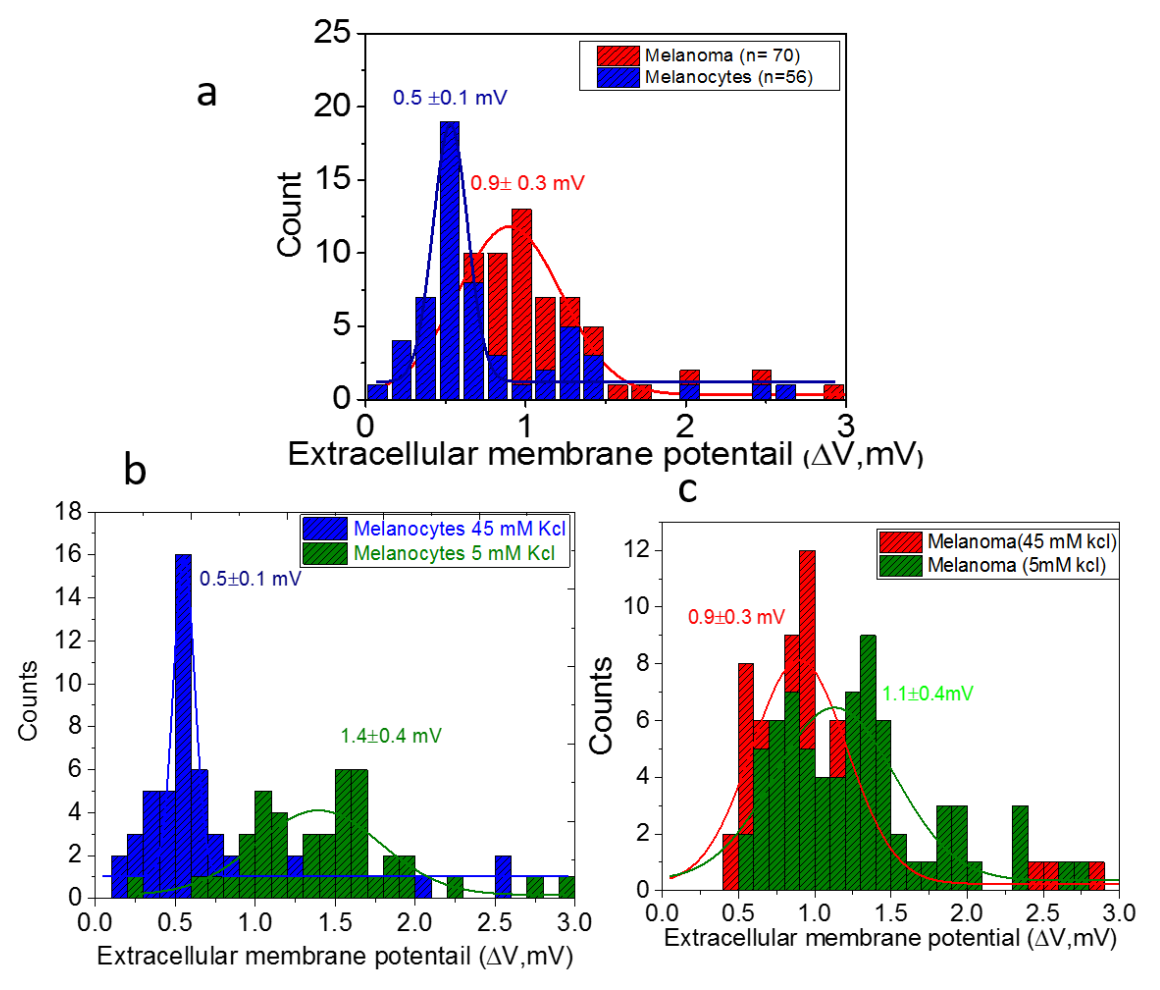

Figure 6.3. Extracellular membrane potential recorded by P-SICM with fixed point measurement technique. Histograms of extracellular membrane potential of (a) Melanoma (B16) (red) and melanocytes (Mela-A) (blue) recorded in $45 \mathrm{mM}$ outside potassium concentration.(b)melanocytes (Mela-A) recorded in $5 \mathrm{mM}$ (blue) and $45 \mathrm{mM}$ (green) outside potassium concentration. (c) melanoma (B16) recorded in $5 \mathrm{mM}$ (red) and $45 \mathrm{mM}$ (green) outside potassium concentration. The red, blue and green solid lines are Gaussian fits to the histograms. The mean value was $0.5 \pm 0.1 \mathrm{mV}$ and $0.9 \pm 0.3 \mathrm{mV}$ for Mela-A and B16 cells respectively in $45 \mathrm{mM}$ outside kcl concentration. The mean value became $1.4 \pm$ $0.4 \mathrm{mV}$ and $1.1 \pm 0.4 \mathrm{mV}$ for Mela-A and B16 respectively in $5 \mathrm{mM}$ outside $\mathrm{kcl}$. .The error bar is the standard deviation of the Gaussian fits.

\subsection{2: Potential Measurement by Mapping Certain Area of Living Cell}

For simultaneously mapping of the topography and extracellular membrane potential distribution, Approach-Retract Scanning (ARS) mode (or so-called hopping mode) ${ }^{20}$ was used to control the theta nanopipette position during imaging. The bandwidth of the current amplifier was set at $10 \mathrm{~Hz}$ to reduce the current noise during imaging and 
potential were recorded with $10 \mathrm{X}$ gain. All the measurements were performed at $37^{\circ}$ temperature.

Besides the fixed point measurement, we have also mapped the potential distribution and topography images of Mela -A cell with our potentiometric SICM. Our result revealed the different micro-domains of extracellular membrane potential of the living cell membranes (Figure $6.4(\mathrm{~b}, \mathrm{~d})$ ). Living mela-A was imaged in 5mM outside potassium concentration using hopping mode. $1 \%$ reduced set point current was used to control probe- sample separation during imaging. Figure 6.4 (a) is the topography image, and $6.4(\mathrm{~b})$ is potential imaging recorded at the same time. 6.4 (c) is enhanced color topography image which makes even small features in the sample clearly visible. Blue color represents flat and low height region, and the orange color represents high height region.In enhanced color topography we can also see collagen pattern. In the potential image, several potential microdomains with different potentials can be seen. Collagen fiber has zero net charges within $\mathrm{pH}$ range $7-8$. In overall cell membrane have a negative potential. Images were recorded at living cell buffer at $\mathrm{pH}$ 7.4. In potential images shown figure 6.4(b \& d), potential distribution was higher on the collagen than on the cell surface.We have used the absolute value of the potential reading color scale of the potential image. The extracellular potential variation within the single cell was in the range of less than $10 \mathrm{mV}$. The potential distribution recorded from the fixed point measurement and potential imaging are in the same range. It should be noted that extracellular membrane potential is smaller than the cross membrane potential which is in the range of $-40 \mathrm{mV}$ to $80 \mathrm{mV}$. 

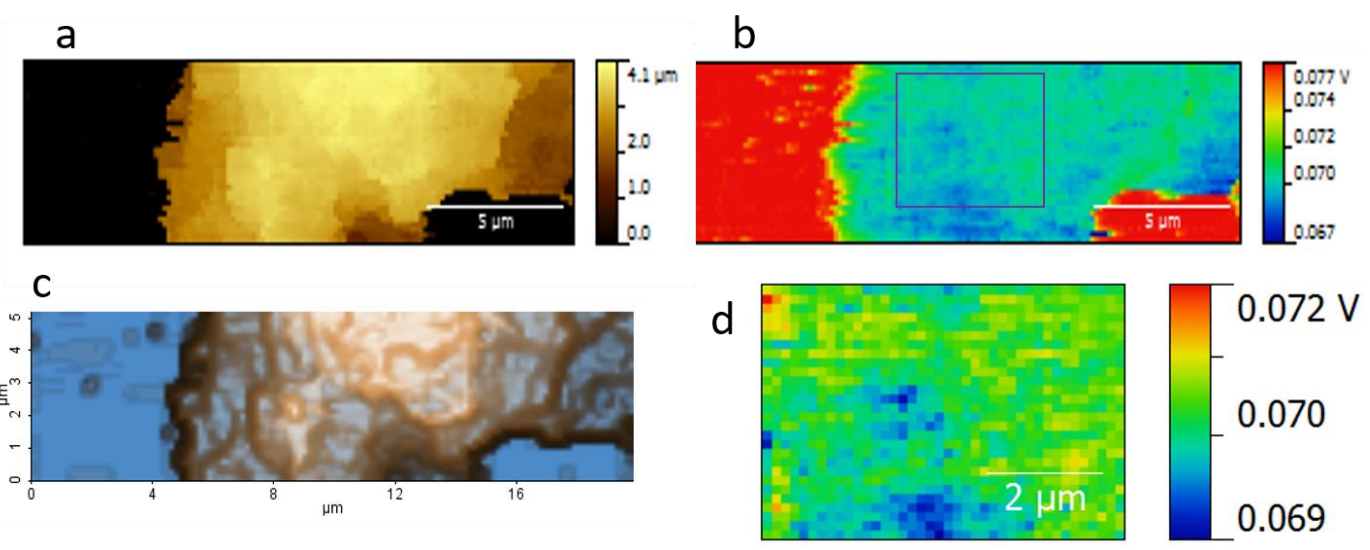

Figure 6.4 Simultaneous recorded (a) topography and (b) potential image of living melanoma cell with P-SICM. (c) Enhanced color topography image (d) Zoom in the image of the potential image inside a rectangular area.

\section{4: Finite Element Based Numerical Simulations}

The potential distribution pattern was investigated using a FEM for solving Poisson (P) and Nernst-Planck equations. A 2D axis symmetry geometry model was created to mimic the potentiometric SICM setup with theta pipette. PNP equations were fully coupled and solved using boundary conditions matching the expected experimental conditions. Fluidic flow term was not included and the system was assumed at a steady state to simplify the simulation. COMSOL Multiphysics 5.2 with $\mathrm{AC} / \mathrm{DC}$ and Chemical Reaction Engineering modules were used for the FEM simulation. The whole computation domain was discretized into free triangular elements, and rigorous mesh refinements were adopted during simulations.

What is the potential detection mechanism for the different charged substrates? We have carried out FEM simulations to understand the potential detection mechanism in this system.. Obviously, the potential of a charged object can be detected based on the direct potential or charge sensing mechanism: the presence of a charged object alters the potential 
of a nearby potential probe. However, the sensing range of this mechanism is very short in ionic solution because of the charge screening effect.We have carried out the simulations on positive $25 \mathrm{mC} / \mathrm{m}^{2},-25 \mathrm{mC} / \mathrm{m}^{2}$ negative and $0 \mathrm{mC} / \mathrm{m}^{2}$ charged substrate by varying the vertical position of the potential probe. The data were plotted as probe-sample distance versus measured potential because of different charged substrate. The result from simulation also follows the same trend like in experimental result with the positive and negative charged substrate as shown in figure 6.5. The measured potential value on the $25 \mathrm{mC} / \mathrm{m}^{2}$ charged substrate is much bigger than the $+25 \mathrm{mC} / \mathrm{m}^{2}$ charged substrate. The measured potential value on $0 \mathrm{mC} / \mathrm{m}^{2}$ charged substrate is in between of negative and positive charged substrate.

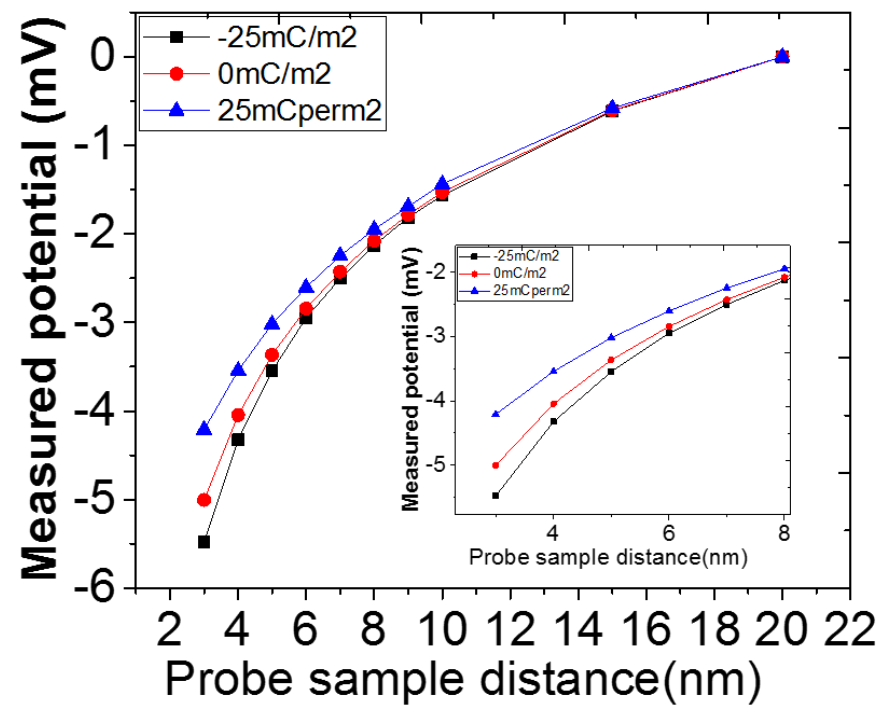

Figure 6.5: FEM simulation result of measured potential with respect to the vertical position of the potential probe from different surface charged substrate. Figure inset is the zoomed in the plot of the original plot. 


\subsection{Conclusion:}

In summary, Scanning ion conductance microscopy has been shown to be a powerful technique for quantitative measurement of extracellular membrane potential distribution of a single living cell. Simultaneous mapping of potential distribution along with its topography imaging of living cell membranes is reported for the first time. We are able to measure the significant potential difference on the positively charged substrate and negatively charged substrate from both the experiment and finite element simulation method. Furthermore, significant potential difference has been found between the normal (Mela-A) and cancerous (B16) cells. We also observed normal cell react more with the outside environment change than in cancer cell.

\section{5: References}

1. Adams, D. \& Levin, M. Cell and Tissue Research 2012, 1.

2. Adams, D.S. \& Levin, M. Cold Spring Harbor Protocols 2012, 2012, pdb.prot067702.

3. Levin, M. \& Stevenson, C.G. Annual Review of Biomedical Engineering 2012, 14, 295.

4. McCaig, C.D., Rajnicek, A.M., Song, B. \& Zhao, M. Physiol Rev 2005, 85, 943.

5. Huang, X. \& Jan, L.Y. J Cell Biol 2014, 206, 151.

6. Robinson, K.R. \& Messerli, M.A. Bioessays 2003, 25, 759.

7. Hansma, P., Drake, B., Marti, O., Gould, S. \& Prater, C. Science 1989, 243, 641.

8. Prater, C.B., Hansma, P.K., Tortonese, M. \& Quate, C.F. Review of Scientific Instruments 1991, 62, 2634. 
9. Novak, P., Shevchuk, A., Ruenraroengsak, P., Miragoli, M., Thorley, A.J., Klenerman, D., Lab, M.J., Tetley, T.D., Gorelik, J. \& Korchev, Y.E. Nano letters 2014, 14, 1202.

10. Robertson, G.A. Circulation research 2013, 112, 1088.

11. Lazenby, R.A., McKelvey, K. \& Unwin, P.R. Analytical Chemistry 2013, 85, 2937.

12. Shen, M., Ishimatsu, R., Kim, J. \& Amemiya, S. Journal of the American Chemical Society 2012, 134, 9856.

13. Momotenko, D., McKelvey, K., Kang, M., Meloni, G.N. \& Unwin, P.R. Anal Chem 2016, 88, 2838.

14. Perry, D., Paulose Nadappuram, B., Momotenko, D., Voyias, P.D., Page, A., Tripathi, G., Frenguelli, B.G. \& Unwin, P.R. Journal of the American Chemical Society 2016, 138, 3152 .

15. Zhou, Y., Bright, L.K., Shi, W., Aspinwall, C.A. \& Baker, L.A. Langmuir 2014, 30,15351 .

16. Klausen, L.H., Fuhs, T. \& Dong, M. Nature Communications 2016, 7, 12447.

17. Zhou, Y., Chen, C.-C., Weber, A.E., Zhou, L. \& Baker, L.A. Langmuir 2014, 30, 5669.

18. Sviderskaya, E.V., Hill, S.P., Evans-Whipp, T.J., Chin, L., Orlow, S.J., Easty, D.J., Cheong, S.C., Beach, D., DePinho, R.A. \& Bennett, D.C. J Natl Cancer Inst 2002, 94, 446.

19. Zhou, Y., Wong, C.-O., Cho, K.-j., van der Hoeven, D., Liang, H., Thakur, D.P., Luo, J., Babic, M., Zinsmaier, K.E., Zhu, M.X., Hu, H., Venkatachalam, K. \& Hancock, J.F. Science 2015, 349, 873.

20. Novak, P., Li, C., Shevchuk, A.I., Stepanyan, R., Caldwell, M., Hughes, S., Smart, T.G., Gorelik, J., Ostanin, V.P., Lab, M.J., Moss, G.W., Frolenkov, G.I., Klenerman, D. \& Korchev, Y.E. Nat Methods 2009, 6, 279. 


\section{CHAPTER 7: SUMMARY AND FUTURE RESEARCH}

\section{1: Summary of Results}

In summary, my dissertation demonstrates the capability of SICM for the single cell imaging and analysis. Morphology change of HeLa cell membrane induced by water soluble and fluorescent conjugated polymer nanoparticles (CPNs) was studied first time by SCIM. The capability of SICM in the detailed study of the attachment of cationic CPNs to the cell surface, corresponding morphology change of the cell surface, and the effect of the following endocytosis was demonstrated. Results revealed that CPNs formed the cluster at the cell surface before entering the cells. These clusters enhance the attachments of CPNs to the cell surface. The physicochemical properties (hydrophobicity and positive charge) of polymer CPNs also affect the attachments and aggregations of CPNs to the cell surface. After CPN attachment, the cells actively internalize CPNs. The CPN attachment is the first step of CPN endocytosis. Therefore, the careful design of the physicochemical properties of $\mathrm{CPN}$ to promote cellular attachment can significantly impact the CPN endocytosis efficiency.

Conventional SICM has been upgraded and validated for the mapping of topography and potential distributions of the single living cell membrane simultaneously. In the first step, multifunctional nanopipette, which contains both nanopore and carbon nanoelectrode $(\mathrm{CNE})$ at the nanopipette tip was developed and characterized to enhance the sensitivity of the potential measurement. To demonstrate the capability of the multifunctional nanopipette, charged gold nanoparticles (GNP) was used as the model sample. The capability of the multifunctional nanopipette for simultaneous detection of ionic current and local electrical potential changes during translocation GNPs was clearly 
demonstrated. The CNE functions as a local potential probe. We have shown that CNE can detect the local potential change during translocation of a single GNP as well as collective potential change due to a cluster of GNPs outside the nanopore entrance. From the potential change, we can also have the insight of motion of GNPs before entering the nanopore. Finite element based numerical simulation also supported the experimental results. Simulations further verified the potential sensing mechanism of the floating carbon electrode, which was found to be very sensitive to the surface charge variation near the pore mouth.

In the second step, the SICM was utilized for the measurement of extracellular potential distributions of a living normal skin cell, melanocytes (Mela-A) and a cancerous skin cell, melanoma (B16) by using SICM with dual barrel probe. We observed not only the spatial variation but also the mean magnitude of the extracellular potential of the MelaA cells was bigger than those of B16 cells. We also found the change in membrane potential of B16 cells is small with a change of potassium ion concentration in bath solutions than mela-A cells. We were also able to map the potential image along with its topography image of the living cell membrane with hopping mode As compared to the voltage reporter dye based fluorescence microscope method, SICM can achieve quantitative potential measurements of non-labeled living cell membranes with higher spatial resolution.

Our results demonstrate that we have established a new imaging technique for measuring extracellular membrane potential distribution of living cell membrane along with its topography images. This new imaging technique can help biologist to explore the extracellular potential distribution of varieties of cells quantitatively. These studies will 
have impacts on several biomedical applications such as regenerative repair and cancer treatment.

In addition, shape and size of nanoparticles synthesized for drug delivery have been characterized with the AFM. Formation of core-shell nanoparticles by complexing a semiflexible PPB with a linear polysaccharide, HA was clearly observed by AFM imaging. Since the size and shape of nanomaterials significantly influence labeling and delivery efficiency of biological substances, the synthesis of core-shell nanoparticle will contribute to novel biomaterials especially for drug delivery.

\section{2: Future Research}

\subsection{1: Potential and Topography Imaging of the Living Cell Membranes with SICM}

The main goal of my dissertation is to develop the multifunctional SICM for the study and analysis of the single living cell.We have developed and demonstrated the capability of the SICM for simultaneous recording of the topography and potential distribution of the living cell membranes, but the experimental setup and imaging condition further need to be optimized to get high-resolution potential images. Multifunctional nanopipette (nanopore/carbon electrode) will be used as a probe instead of bare theta pipette. Improvement of the living cell imaging environment will help to increase working hours during the experiment. Several potential images of the both Mela $-\mathrm{A}$ and B16 will be collected by changing the outside buffer environment and compare the potential distributions. Current, and potential approach curves will be analyzed in detail to get the quantitative potential measurement at a different height from the cell. Not only Mela-A and B16 other cell lines like HeLa cell and PC12 also will be imaged and analyzed. 


\subsection{2: Potential Approach Curve Spectroscopy Analysis}

The current and potential approach curves during simultaneous mapping of topography and potential will be collected. The potential and current approach curves will be analyzed with LabVIEW. The spatial resolution of the potential image at a different working distance (probe-sample distance) will be examined. Detailed analysis of the potential approach curves will help us to differentiate the surface charge effect and the ion flux effect in the measured potential distribution of the living cells. Based on Potential Vs distance $(\mathrm{V}-\mathrm{z})$ curves, quantitative understanding of the potential change with working distance will be clear. The 3D potential images will be generated

\subsection{3: Finite Element Based Numerical Simulations}

Finite element based numerical simulations will be carried out to interpret the SICM results. Following the previous reports ${ }^{1-4}$ finite element based numerical simulations will be carried out using COMSOL. Especially, the effect of the new nanopipette geometries to the ionic current and electric potential distribution will be investigated. Furthermore, the sensitivity of potential measurement on the different charged substrate with the variation of the distance will be simulated. These simulations will help us to interpret our experimental result better.

\section{3: Multifunctional Nanopipette for the Studies of Nanoparticles}

The high precision quantitative measurements are limited by the large variations in the geometry of multifunctional nanopipettes. The fabrication of multifunctional nanopipette will be further improved and optimized. Transmission electron microscopy will be used for the better characterization of nanopore/nanoelectrode ${ }^{4}$, which will enable more quantitative measurements of NPs. Multifunctional nanopipette will be further used 
for analysis of several other nanoparticles like virus nanoparticles (VNPs) and the polystyrene beads which have around 10 times higher surface charge density in compare to the GNPs. Biomolecules such as DNA and proteins will also be analyzed with our nanopore /nanoelectrode.

7.4: Topography and Potential Imaging of Hole Formation on the Cell Membrane by SICM.

The interaction between the conjugated polymer nanoparticles (CPNs) and the cell membrane will be further characterized by the SICM. Effect of CPNs side chain end group in the morphology of cell membrane will be studied. A future project will be the study of the hole formation on the cell membranes during endocytosis of guanidine nanoparticles. The guanidine nanoparticles are also the CPNs whose side chain is slightly changed from the previous CPNs structure (PPB) that we used in Chapter 4. The structure of the guanidine nanoparticles has shown in Figure 7.1(c) where the red circle marks side chain. Our hypothesis will be this typical side chain of the CPNs will form the nanometer-sized hole on the cell membranes. The hole formations on the fixed HeLa cell membrane with the 1hour treatment of the guanidine nanoparticles has already been revealed by the topography images of SICM which has shown in figure 7.1(a and b). The height profile in Figure 7.1(d) clearly shows the downward spikes with depth around 600nm to 1 micron. Not only the topography images but also the potential images of hole formations on the HeLa cell membranes will be acquired and analyzed. Both fixed and living HeLa cell membranes will be imaged by incubating with the guanidine nanoparticles. The experiment will be designed by varying the incubation time as well as the concentration of the guanidine with 
the cell samples. As explained in 7.2.1 potential and current approach curves will be generated, and 3D potential images will be generated.

a

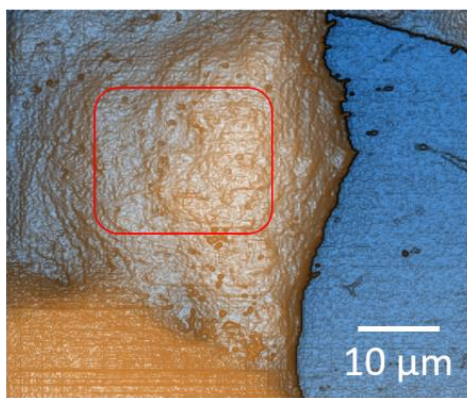

b

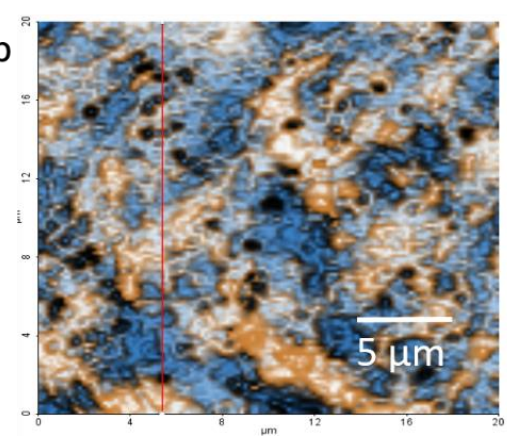

c
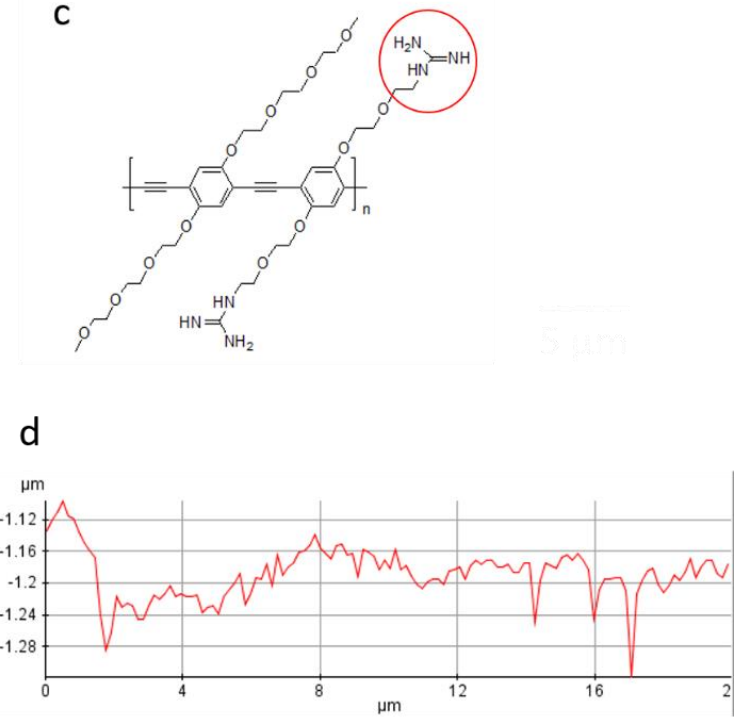

Figure 7.1 (a) Enhanced color topography image of fixed HeLa cell treated with the $10 \mu \mathrm{M}$ guanidine for $1 \mathrm{hr}$. (b) Zoomed in images of the Figure (a) enclosed inside the red rectangular area. (c) The structure of the guanidinium CPN where red circle focuses the side chain. (d) The height profile across the red solid line in Figure b. The cell was fixed in $4 \%$ paraformaldehyde.

\section{5: Optical Fluorescence Microscopy Imaging}

An optical fluorescence microscopy technique will be added in the SICM set up. In the beginning, epifluorescence microscopy will be added. The required accessories such as an objective lens, dichromatic mirror, excitation, and emission filter will be either added or upgraded in the SICM set up for the simultaneous detection of the fluorescence images, and SICM topography, and potential images of CPN treated fixed and living HeLa cells at different conditions. 


\section{6: References}

1. Lan, W.-J., Holden, D.A., Zhang, B. \& White, H.S. Analytical Chemistry 2011, 83, 3840 .

2. Lan, W.-J., Kubeil, C., Xiong, J.-W., Bund, A. \& White, H.S. The Journal of Physical Chemistry C 2014, 118, 2726.

3. Tiwari, P.B., Astudillo, L., Miksovska, J., Wang, X., Li, W., Darici, Y. \& He, J. Nanoscale 2014, 6, 10255.

4. Perry, D., Momotenko, D., Lazenby, R.A., Kang, M. \& Unwin, P.R. Analytical Chemistry 2016, 88, 5523. 
VITA

NAMUNA PANDAY

EDUCATION

2011-2017

Florida International University, Miami, FL, Ph.D. Candidate (Physics).

2006-2008

Tribhuvan University, Kathmandu, Nepal, M. Sc. (Physics).

2003-2006

Tribhuvan University, Kathmandu, Nepal, B. Sc. (Physics).

2017

First Prize Award, Graduate Student Research Competition, Department of Physics, Florida International University.

2016

Second Prize Award, NanoFlorida, UCF, on the oral presentation.

2016-2017

Dissertation Year Fellowship, Florida International University.

2015-2016

Research Assistantship, Florida International University.

2016

Second Prize Award, Graduate Student Research Competition, Department of Physics, Florida International University.

2015

Second Prize Award, Graduate Student Research Competition, Department of Physics, Florida International University.

2014

First Prize Award, Park System Atomic Force Microscopy Image Contest, Santa Clara, CA.

2013-2017

Trained graduate and undergraduate students to handle AFM \& SICM. Supervised middle school teachers and high school students for their summer research projects on nanopipettes, AFM, SICM, Florida International University, FL.

2014-2015 Co-instructor in online Physics course. Experienced on managing course material in website, delivering online homework and lectures, Florida International University, FL.

2011-2014 Lab instructor in general Physics lab (equipped for activities in Mechanics, Optics, Electricity and Magnetism etc.), Florida International University, FL. 


\section{PUBLICATIONS AND PRESENTATIONS:}

1. Namuna Panday, Gongming Qian, Xuewen Wang, Shuai Chang, Popular Pandey and Jin He, Simultaneous ionic and potential detection of nanoparticles by a multifunctional nanopipette ", ACS Nano, 11237-11248(2016).

2. Yuping Shan, Namuna Panday, Yong Myoung, Megan Twomey, Xuewen Wang, Wenzhi Li, Emrah Celik, Vincent Moy, Hongda Wang, Joong Ho-Moon, Jin He, Scanning Ion Conductance Microscopic Study for Cel-lular Uptake of Cationic Conjugated Polymer Nanoparticles", Macromolecular Bioscience, 599-607(2016).

3. Namuna Panday, Jin He, Scanning Ion Conductance Microscopy for single cell imaging and analysis", Advanced Science Engineering and medicine, 10581065(2015).

4. Megan Twomey, Yoonmi Na, Zahilyn Roche, Eladio Mendez, Namuna Panday, Jin He, Joong Ho Moon, Fabrication of Core Shell Nanoparticles via Controlled Aggregation of Semiexible Conjugated Polymer and Hyaluronic Acid", Macromolecules, 6374-6378(2013).

5. "Multifunctional nanopipette for simultaneous ionic current and potential detection of nanoparticles". Namuna Panday, APS Meeting, March 15, 2017, New Orleans, Louisiana.

6. "Extracellular Membrane Potential Measurement of Single Living Cells With Scanning Ion Conductance Microscopy ". Namuna Panday, BPS Meeting, Feb 15, 2017, New Orleans, Louisiana.

7. "Multifunctional nanopipette for simultaneous ionic current and potential detection of nanoparticles". Namuna Panday, Nanoflorida Conference, September 26, 2016, Orlando, Florida.

8. "Scanning Ion Conductance Microscopy for living cell membrane potential measurement". Namuna Panday, APS Meeting, March 16, 2016, Baltimore, Maryland.

9. "Morphology Change of HeLa Cell Membrane induced by the endocytosis of conjugated polymer nanoparticles". Namuna Panday, Florida Chapter of the AVS Science and Technology Society (FLAVS) meeting, March 3, 2014. 